\title{
The Milky Way Tomography with SDSS. II. Stellar Metallicity
}

\section{Citation}

Ivezic, Zeljko, Branimir Sesar, Mario Juric, Nicholas Bond, Julianne Dalcanton, Constance M. Rockosi, Brian Yanny, et al. 2008. "The Milky Way Tomography with SDSS. II. Stellar Metallicity." The Astrophysical Journal 684 (1) (September): 287-325. doi:10.1086/589678.

\section{Published Version}

doi:10.1086/589678

\section{Permanent link}

http://nrs.harvard.edu/urn-3:HUL.InstRepos:33462885

\section{Terms of Use}

This article was downloaded from Harvard University's DASH repository, and is made available under the terms and conditions applicable to Other Posted Material, as set forth at http:// nrs.harvard.edu/urn-3:HUL.InstRepos:dash.current.terms-of-use\#LAA

\section{Share Your Story}

The Harvard community has made this article openly available.

Please share how this access benefits you. Submit a story.

Accessibility 


\section{THE MILKY WAY TOMOGRAPHY WITH SDSS. II. STELLAR METALLICITY}

Željko Ivezić, ${ }^{1}$ Branimir Sesar, ${ }^{1}{ }_{6}$ Mario Jurić, ${ }^{2}$ Nicholas Bond, ${ }^{3}$ Julianne Dalcanton, ${ }^{1}$ Constance M. Rockosi, ${ }^{4}$ Brian Yanny, ${ }^{5}$ Heidi J. Newberg, ${ }^{6}$ Timothy C. Beers, ${ }^{7}$ Carlos Allende Prieto, ${ }^{8}$ Ron Wilhelm, ${ }^{9}$ Young Sun Lee, ${ }^{7}$ Thirupathi Sivarani, ${ }^{7}$ John E. Norris, ${ }^{10}$ Coryn A. L. Bailer-Jones, ${ }^{11}$ Paola Re Fiorentin, ${ }^{11,12}$ David Schlegel, ${ }^{13}$ Alan Uomoto, ${ }^{14}$ Robert H. Lupton, ${ }^{3}$ Gillian R. Knapp, ${ }^{3}$ James E. Gunn, ${ }^{3}$ Kevin R. Covey, ${ }^{15}$ J. Allyn Smith, ${ }^{16}$ Gajus Miknaitis, ${ }^{5}$ Mamoru Doi, ${ }^{17}$ Masayuki Tanaka, ${ }^{18}$ Masataka Fukugita, ${ }^{19}$ Steve Kent, ${ }^{5}$ Douglas Finkbeiner, ${ }^{15}$ Jeffrey A. Munn, ${ }^{20}$ Jeffrey R. Pier, ${ }^{20}$ Tom Quinn, ${ }^{1}$ Suzanne Hawley, ${ }_{1}$ Scott Anderson, ${ }^{1}$ Furea Kiuchi, ${ }^{1}$ Alex Chen, ${ }^{1}$ James Bushong, ${ }^{1}$ Harkirat Sohi, ${ }^{1}$ Daryl Haggard, ${ }^{1}$ Amy Kimball, ${ }^{1}$ John Barentine, ${ }^{21}$ Howard Brewington, ${ }^{21}$ Mike Harvanek, ${ }^{21}$ Scott Kleinman, ${ }^{21}$ Jurek Krzesinski, ${ }^{21}$ Dan Long, $^{21}$ Atsuko Nitta, ${ }^{21}$ Stephanie Snedden, ${ }^{21}$ Brian Lee, ${ }^{13}$ Hugh Harris, ${ }^{20}$ Jonathan Brinkmann, ${ }^{21}$ Donald P. Schneider, ${ }^{22}$ and Donald G. York ${ }^{23}$ Received 2007 October 4; accepted 2008 April 22

\section{ABSTRACT}

Using effective temperature and metallicity derived from SDSS spectra for $\sim 60,000 \mathrm{~F}$ - and G-type main-sequence stars $(0.2<g-r<0.6)$, we develop polynomial models for estimating these parameters from the SDSS $u-g$ and $g-r$ colors. These photometric estimates have similar error properties as those determined from SDSS spectra. We apply this method to SDSS photometric data for over 2 million F/G stars and measure the unbiased metallicity distribution for a complete volume-limited sample of stars at distances between $500 \mathrm{pc}$ and $8 \mathrm{kpc}$. The metallicity distribution can be exquisitely modeled using two components with a spatially varying number ratio, which correspond to disk and halo. The two components also possess the kinematics expected for disk and halo stars. The metallicity of the halo component is spatially invariant, while the median disk metallicity smoothly decreases with distance from the Galactic plane from -0.6 at 500 pc to -0.8 beyond several kiloparsecs. The absence of a correlation between metallicity and kinematics for disk stars is in a conflict with the traditional decomposition in terms of thin and thick disks. We detect coherent substructures in the kinematics-metallicity space, such as the Monoceros stream, which rotates faster than the LSR, and has a median metallicity of $[\mathrm{Fe} / \mathrm{H}]=-0.95$, with an rms scatter of only $\sim 0.15$ dex. We extrapolate our results to the performance expected from the Large Synoptic Survey Telescope (LSST) and estimate that LSST will obtain metallicity measurements accurate to 0.2 dex or better, with proper-motion measurements ac-

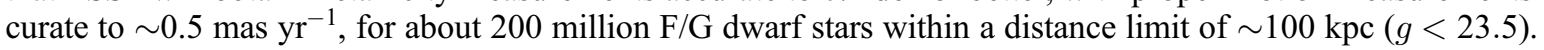

Subject headings: Galaxy: halo — Galaxy: kinematics and dynamics — Galaxy: stellar content —

Galaxy: structure — methods: data analysis — stars: statistics

Online material: color figures

\section{INTRODUCTION}

A major objective of modern astrophysics is to understand when and how galaxies formed, and how they have evolved since then. Our own Galaxy, the Milky Way, provides a unique opportunity to study a galaxy in great detail by measuring and analyzing the properties of a large number of individual stars.

The formation of galaxies like the Milky Way was long thought to be a steady process leading to a smooth distribution

${ }^{1}$ Department of Astronomy, University of Washington, Seattle, WA 98195.

2 Institute for Advanced Study, Princeton, NJ 08540.

3 Princeton University Observatory, Princeton, NJ 08544.

${ }^{4}$ University of California, Santa Cruz, CA 95060.

5 Fermi National Accelerator Laboratory, Batavia, IL 60510.

${ }^{6}$ Department of Physics, Applied Physics, and Astronomy, Rensselaer Polytechnic Institute, Troy, NY 12180.

7 Department of Physics and Astronomy, Center for the Study of Cosmic Evolution (CSCE) and Joint Institute for Nuclear Astrophysics (JINA), Michigan State University, East Lansing, MI 48824.

${ }^{8}$ McDonald Observatory and Department of Astronomy, University of Texas, Austin, TX 78712.

9 Department of Physics, Texas Tech University, Lubbock, TX 79409.

${ }_{10}$ Research School of Astronomy and Astrophysics, Australian National University, Weston ACT 2611, Australia.

${ }_{11}$ Max Planck Institut für Astronomie, 69117 Heidelberg, Germany.

12 Department of Physics, University of Ljubljana, 1000 Ljubljana, Slovenia.

${ }^{13}$ Lawrence Berkeley National Laboratory, Berkeley, CA 94720.

14 Department of Physics and Astronomy, John Hopkins University, Baltimore, MD 21218 . of stars, with this standard view exemplified by the Bahcall \& Soneira (1980) and Gilmore et al. (1989) models and described in detail by, e.g., Majewski (1993). In these smooth models, the spatial distribution of stars in the Milky Way is usually ${ }^{24}$ modeled by three discrete components described using relatively simple analytic expressions: the thin disk, the thick disk, and the halo. However, recent discoveries of complex substructure in the distribution and kinematics of the Milky Way's stars (e.g., Ivezić et al. 2000; Yanny et al. 2000; Vivas et al. 2001; Newberg et al. 2002; Gilmore et al. 2002; Majewski et al. 2003; Duffau et al. 2006; Vivas \& Zinn 2006; Grillmair 2006a, 2006b; Belokurov

15 Harvard-Smithsonian Center for Astrophysics, Cambridge, MA 02138.

16 Department of Physics and Astronomy, Austin Peay State University, Clarksville, TN 37044.

17 Institute of Astronomy, University of Tokyo, 2-21-1 Osawa, Mitaka, Tokyo 181-0015, Japan.

18 Department of Astronomy, Graduate School of Science, University of Tokyo, Bunkyo-ku, Tokyo 113-0033, Japan.

${ }^{19}$ Institute for Cosmic Ray Research, University of Tokyo, Kashiwa, Chiba, Japan.

${ }^{20}$ US Naval Observatory, Flagstaff Station, Flagstaff, AZ 86002.

21 Apache Point Observatory, Sunspot, NM 88349-0059.

22 Department of Astronomy and Astrophysics, Pennsylvania State University, University Park, PA 16802.

${ }_{23}$ University of Chicago, Astronomy and Astrophysics Center, and Enrico Fermi Institute, Chicago, IL 60637.

24 Infrared data toward the Galactic center require addition of a bulge and a stellar bar (e.g., Weinberg 1992; Jackson et al. 2002 and references therein). 
et al. 2006, 2007; Bell et al. 2007; Jurić et al. 2008, hereafter J08) have deeply shaken this standard view. Unlike those smooth models that involve simple components, the new data indicate many irregular structures, such as the Sgr dwarf tidal stream in the halo and the Monoceros stream closer to the Galactic plane. These recent developments, based on accurate large-area surveys, have made it abundantly clear that the Milky Way is a complex and dynamical structure that is still being shaped by the infall (merging) of neighboring smaller galaxies.

Numerical simulations suggest that this merger process plays a crucial role in setting the structure and motions of stars within galaxies and is a generic feature of current cosmological models (e.g., Helmi et al. 1999; Springel \& Hernquist 2003; Bullock \& Johnston 2005). Since the individual stars that make up the stellar populations in the Milky Way can be studied in great detail, their characterization will provide clues about the galaxy merging process that cannot be extracted from observations of distant galaxies (e.g., Abadi et al. 2003; Brook et al. 2004 and references therein).

The three presumably discrete Milky Way components differ not only in their spatial profiles but also in the detailed distributions of their kinematics and metallicity (e.g., Majewski 1993; Ojha et al. 1996; Freeman \& Bland-Hawthorn 2002; Robin et al. 2003; Wyse 2006 and references therein). The thin disk, with a scale height of $\sim 300 \mathrm{pc}$, has a vertical velocity dispersion of $\sigma_{z} \sim 20 \mathrm{~km} \mathrm{~s}^{-1}$, while the thick disk, with a scale height of $\sim 1000 \mathrm{pc}$, is somewhat warmer $\left(\sigma_{z} \sim 40 \mathrm{~km} \mathrm{~s}^{-1}\right)$ and older, has a lower average metallicity $\left(\left[Z / Z_{\odot}\right] \sim-0.7\right.$; e.g., Gilmore \& Wyse 1985), and has enhanced $\alpha$-element abundances (e.g., Fuhrmann 2004; Bensby et al. 2003; Feltzing 2006; Reddy et al. 2006; Ramírez et al. 2007). In contrast, the halo is composed mainly of low-metallicity stars $\left(\left[Z / Z_{\odot}\right]<-1.0\right.$; e.g., Ryan \& Norris 1991$)$ and has little or no net rotation. Hence, in addition to their spatial profiles, the main differences between these components are in their rotational velocity distributions, velocity dispersions, and metallicity distributions.

We note that a recent study by Carollo et al. (2007), based on a sample of over 20,000 calibration stars with available spectra from SDSS Data Release 5, has demonstrated that "the halo" of the Galaxy is likely to comprise two distinct components. According to these authors, the inner halo component dominates the population of halo stars found at distances up to $10-15 \mathrm{kpc}$ from the Galactic center (including the solar neighborhood), and an outer halo component dominates in the regions beyond 15$20 \mathrm{kpc}$. The inner halo stars are nonspherically distributed about the center of the Galaxy, with an inferred axis ratio of $\sim 0.6$, while the outer halo comprises stars that exhibit a much more spherical spatial distribution. Our present study only reaches to $8 \mathrm{kpc}$ from the Sun and hence is likely to be dominated by inner halo stars. Therefore, for the purpose of the present paper, we assume a single-component halo.

Despite the significant progress that has been made over the years, we still cannot answer some simple questions, such as the following: Are the exponential profiles used to describe the spatial profiles of thin and thick disks an oversimplification? Why do estimates for thick-disk scale height differ by a factor of several between different studies (for a discussion see Siegel et al. 2002; J08)? Is the transition between thin and thick disks in metallicity and kinematics abrupt or continuous? Is there a largescale metallicity gradient in the thick disk and halo? Does the disk scale length depend on metallicity? Can large spatial substructures be traced in kinematic and metallicity spaces?

To reliably answer these and similar questions, a data set needs to be voluminous (to enable sufficient spatial, kinematic, and met- allicity resolution), diverse (accurate distance and metallicity estimates, as well as radial velocity and proper-motion measurements, are required), and faint (to probe a significant fraction of the Galaxy). Modern sky surveys, such as the Sloan Digital Sky Survey (SDSS; York et al. 2000), with its imaging and spectroscopic components, and the Two Micron All Sky Survey (Skrutskie et al. 2006), with its all-sky coverage, have recently provided such data sets.

Most studies of the Milky Way structure can be described as investigations of the stellar distribution in the nine-dimensional space spanned by the three spatial coordinates, three velocity components, and three main stellar parameters (luminosity, effective temperature, and metallicity). Depending on the quality, diversity, and quantity of data, such studies typically concentrate on only a limited region of this space (e.g., the nearby solar neighborhood, pencil beam surveys, kinematically biased surveys), or consider only marginal distributions of selected quantities (e.g., number density of stars irrespective of their metallicity or kinematics, luminosity function determinations, proper-motion surveys without metallicity or radial velocity information). We use the SDSS data to study in detail the stellar distribution in this multidimensional space. We focus on the metallicity distribution of disk and halo stars in this contribution. In companion papers we discuss the spatial distribution of stars (J08) and their kinematics (N. Bond et al. 2008, in preparation, hereafter B08).

In $\S 2$ we use the data for $\sim 60,000$ probable F- and G-type main-sequence stars provided by the SDSS spectroscopic survey to calibrate a method for estimating metallicity from the $u-$ $g$ and $g-r$ colors measured by the SDSS photometric survey. Readers who are not interested in technical aspects of this method may want to skip directly to $\S 3$, where we apply this method to two photometric catalogs constructed using SDSS data. One catalog contains averaged repeated observations and provides sufficiently improved photometric accuracy and depth to study the metallicity distribution all the way to the disk-halo interface at several kiloparsecs from the Galactic plane. The second catalog, based on all SDSS photometric observations to date, covers a wide area and probes a significant fraction of the Galaxy. We summarize and discuss our results in $\S 4$.

\section{DETERMINATION OF STELLAR METALLICITY FROM SDSS PHOTOMETRIC DATA}

The most accurate measurements of stellar metallicity are based on spectroscopic observations. Despite the recent progress in the availability of stellar spectra (e.g., SDSS has recently made publicly available ${ }^{25}$ over 280,000 stellar spectra as a part of its Data Release 6 [DR6]; the proposed extension of SDSS, known as SDSS-III, is capable of providing another several hundred thousand stars with available spectra in the next few years; RAVE ${ }^{26}$ may provide up to a million spectra, primarily thin- and thickdisk stars, over the next few years), the number of stars detected in imaging surveys is vastly larger. In addition to generally providing better sky and depth coverage than spectroscopic surveys, imaging surveys obtain essentially complete flux-limited samples of stars. The simple selection criteria used for the photometric surveys are advantageous when studying Galactic structure, compared to the complex targeting criteria that are used for SDSS stellar spectra (see $\S 2.2$ ). Hence, we use the extant SDSS spectroscopic data to calibrate a method for estimating metallicity from the SDSS imaging data, and we use this calibration to study

\footnotetext{
${ }^{25}$ See http://www.sdss.org/dr6.

${ }^{26}$ See http://www.rave-survey.aip.de/rave.
} 
the metallicity distribution of several million disk and halo stars of the Milky Way.

Stellar metallicity has long been estimated using photometric methods such as the traditional UV excess-based $\delta(U-B)_{0.6}$ method (Wallerstein 1962; Sandage 1969). A blue main-sequence (F and $\mathrm{G}$ type) star's metallicity is correlated with the difference between the star's $U-B$ color and that which would be measured for a metal-rich star with the same $B-V$ color. This correlation is seen in both data (e.g., Carney 1979 and references therein) and detailed stellar models (Kurucz 1979). The Johnson UBV bands are similar to SDSS's $u g r$ bands; thus, it should be possible to derive an analogous method applicable to the SDSS photometric system, as recently attempted by Karaali et al. (2005). However, as they pointed out, their study did not utilize SDSS data, but a somewhat different photometric system. Unfortunately, even small photometric offsets and color terms between different photometric systems may have significant systematic effects on derived metallicities. For example, the SDSS $u$-band measurements are offset from the AB system by $\sim 0.04$ mag (Eisenstein et al. 2006; Holberg \& Bergeron 2006), leading to a metallicity bias of up to 0.2 dex. Here we derive photometric metallicity estimators for the SDSS filter set using SDSS DR6 data. This calibration relies on the large number of stars $(\sim 287,000)$ with a homogeneous set of stellar parameters (effective temperature, metallicity, and gravity) derived from moderate-resolution SDSS spectra (Beers et al. 2006; Allende Prieto et al. 2006, 2007; Lee et al. 2007a, 2007b).

\subsection{An Overview of the Sloan Digital Sky Survey}

The SDSS is a digital photometric and spectroscopic survey that covers about one-quarter of the celestial sphere in the north Galactic cap, as well as a smaller area $\left(\sim 300 \mathrm{deg}^{2}\right)$ but much deeper survey in the southern Galactic hemisphere (Stoughton et al. 2002; Abazajian et al. 2003, 2004, 2005; Adelman-McCarthy et al. 2006). SDSS is using a dedicated $2.5 \mathrm{~m}$ telescope (Gunn et al. $2006)$ to provide homogeneous and deep $(r<22.5)$ photometry in five bandpasses (Fukugita et al. 1996; Gunn et al. 1998; Smith et al. 2002; Hogg et al. 2001; Tucker et al. 2006) repeatable to $0.02 \mathrm{mag}$ (rms scatter for sources not limited by photon statistics; Ivezić et al. 2003) and with a zero-point uncertainty of $\sim 0.02-$ $0.03 \mathrm{mag}$ (Ivezić et al. 2004). The flux densities of detected objects are measured almost simultaneously in five bands $(u, g, r, i$, and $z$ ) with effective wavelengths of $3540,4760,6280,7690$, and $9250 \AA$. The large survey sky coverage will result in photometric measurements for well over 100 million stars and a similar number of galaxies. ${ }^{27}$ The completeness of SDSS catalogs for point sources is $\sim 99.3 \%$ at the bright end and drops to $95 \%$ at magnitudes of 22.1, 22.4, 22.1, 21.2, and 20.3 in $u, g, r, i$, and $z$, respectively. Astrometric positions are accurate to better than $0.1^{\prime \prime}$ per coordinate (rms) for sources with $r<20.5$ (Pier et al. 2003), and the morphological information from the images allows reliable star-galaxy separation to $r \sim 21.5$ (Lupton et al. 2002; Scranton et al. 2002). A compendium of other technical details about SDSS can be found on the SDSS Web site, ${ }^{28}$ which also provides interface for the public data access.

\subsection{SDSS Spectroscopic Survey of Stars}

SDSS spectra are obtained with a pair of dual multiobject fiberfed spectrographs on the same telescope used for the imaging

\footnotetext{
27 The recent DR6 lists photometric data for 287 million unique objects observed in $9583 \mathrm{deg}^{2}$ of sky (Adelman-McCarthy et al. 2008; see http://www.sdss .org/dr6/).

${ }^{28}$ Available at http://www.sdss.org.
}

survey (A. Uomoto et al. 2008, in preparation). Spectroscopic plates have a radius of $1.49^{\circ}$ and take 640 simultaneous spectra, each with wavelength coverage 3800-9200 $\AA$ and spectral resolution of $R \sim 2000$. The signal-to-noise ratio is typically $>4$ pixel $^{-1}$ at $g=20$ but is substantially higher for brighter point sources, such as considered herein.

Targets for the spectroscopic survey are chosen from the SDSS imaging data, described above, based on their colors and morphological properties. ${ }^{29}$ The targets include the following:

1. Galaxies.-A simple flux limit for "main" galaxies, fluxcolor cut for luminous red galaxies (Strauss et al. 2002; Eisenstein et al. 2001).

2. Quasars.-Flux-color cut, matches to FIRST survey (Richards et al. 2002).

3. Nontiled objects (color selected).-Calibration stars (16 per plate), "interesting" stars (hot white dwarfs, brown dwarfs, red dwarfs, red giants, blue horizontal branch stars, carbon stars, cataclysmic variables, central stars of planetary nebulae), sky.

Here, "(non)tiled objects" refer to objects that are not guaranteed a fiber assignment. As an illustration of the fiber assignments, SDSS DR6 contains spectra of 791,000 galaxies, 104,000 quasars, and 287,000 stars.

The spectra are targeted and automatically processed by three pipelines:

1. Target.- Target selection and tiling.

2. Spectro2d.-Extraction of spectra, sky subtraction, wavelength and flux calibration, combination of multiple exposures.

3. Spectrold.-Object classification, redshift determination, measurement of line strengths and line indices.

For each object in the spectroscopic survey, a spectral type, redshift (or radial velocity), and redshift error are determined by matching the measured spectrum to a set of templates. The stellar templates are calibrated using the ELODIE stellar library. Random errors for the radial velocity measurements are a strong function of spectral type and signal-to-noise ratio but are usually $<5 \mathrm{~km} \mathrm{~s}^{-1}$ for stars brighter than $g \sim 18$, rising sharply to $\sim 25 \mathrm{~km} \mathrm{~s}^{-1}$ for stars with $g=20$. Using a sample of multiply observed stars, Pourbaix et al. (2005) estimate that these errors may be underestimated by a factor of $\sim 1.5$. Further technical details about the SDSS spectroscopic survey are available from the SDSS Web site.

\subsection{Stellar Atmospheric Parameter Estimation}

SDSS stellar spectra are of sufficient quality to provide robust and accurate stellar parameters, such as effective temperature, surface gravity, and metallicity (parameterized as $[\mathrm{Fe} / \mathrm{H}]$ ). These parameters are estimated using a variety of methods implemented in an automated pipeline (the SEGUE Stellar Parameter Pipeline [SSPP]; Beers et al. 2006). A detailed discussion of these methods and their performance can be found in Allende Prieto et al. (2006, 2007) and Lee et al. (2007a, 2007b). Based on a comparison with high-resolution abundance determinations, they demonstrate that the combination of spectroscopy and photometry from SDSS is capable of delivering estimates of $T_{\text {eff }}, \log g$, and $[\mathrm{Fe} / \mathrm{H}]$ with external accuracies of $190 \mathrm{~K}(3.2 \%), 0.28 \mathrm{dex}$, and $0.17 \mathrm{dex}$, respectively. These tests indicate that mean systematic errors for $[\mathrm{Fe} / \mathrm{H}]$ and $T_{\text {eff }}$ should not be larger than about $0.2 \mathrm{dex}$ and $100 \mathrm{~K}$

\footnotetext{
29 The recent extension of SDSS, known as SDSS-II, has different targeting priorities. In particular, the subsurvey known as SEGUE (Sloan Extension for Galactic Understanding and Exploration) is optimized for Galactic structure studies.
} 
and may be below 0.1 dex and $75 \mathrm{~K}$ (Lee et al. 2007b). Note that these estimates apply to stars with a wider range of temperatures than we consider in this study.

We use the final adopted values, called teffa and feha in the SDSS sppParams table, which are based on averaging several different methods. A detailed analysis by Lee et al. (2007a, 2007b) demonstrates that systematic metallicity differences between the methods used in averaging do not exceed $\sim 0.1$ dex. A comparison with Galactic open and globular clusters indicates that the adopted metallicity scale systematically overestimates metallicity by $\sim 0.15$ dex for $[\mathrm{Fe} / \mathrm{H}]<-2$ and underestimates metallicity by up to $\sim 0.3$ dex for stars near solar metallicity (the metallicity offsets have been improved recently and are now essentially nil, but for the purpose of this paper, we have made use of a previous version of the SSPP, hence the systematics remains present).

Only a few percent of stars in the SDSS spectroscopic sample are giants. For this reason, we consider only the main-sequence stars, using the selection criteria described below. Although we address photometric estimates of effective temperature, the main goal of this section is to derive a robust and accurate photometric metallicity estimator.

\subsubsection{Sample Selection}

We begin by selecting bright stars from the main stellar locus (Lenz et al. 1998; Fan 1999; Finlator et al. 2000; Smolčić et al. 2004), with colors located in the proper range for the application of the photometric metallicity method (roughly ${ }^{30} 0.4<B-V<$ 0.8; Carney 1979), and from sky regions with modest interstellar dust extinction (SDSS utilizes the Schlegel et al. [1998] maps). The specific criteria applied to 130,620 entries from the so-called sppParams table $\mathrm{e}^{31}$ that have $\log g>0$ are the following:

1. The interstellar extinction in the $r$ band below $0.3:[106,816]$.

2. $14<g<19.5$ : [104,844].

3. $0.2<(g-r)<0.6$ : [75,928].

4. $0.7<(u-g)<2.0$ and $-0.25<(g-r)-0.5(u-g)<$ $0.05:[68,306]$.

5. $-0.2<0.35(g-r)-(r-i)<0.10$ : [66,496].

The numbers in brackets indicate the number of stars left after each selection step.

Using a photometric parallax relation based on observations of globular clusters (see Appendix A for a detailed discussion),

$$
M_{r}(g-i,[\mathrm{Fe} / \mathrm{H}])=M_{r}^{0}(g-i)+\Delta M_{r}([\mathrm{Fe} / \mathrm{H}])
$$

where $\Delta M_{r}([\mathrm{Fe} / \mathrm{H}])$ and $M_{r}^{0}(g-i)$ are given by equations (A2) and (A7), respectively, we further limit the sample to 61,861 stars in the $1-10 \mathrm{kpc}$ distance range. Due to the small $r-i$ color range spanned by $\mathrm{F} / \mathrm{G}$ stars, when comparing results to $\mathrm{J} 08$ it is better to estimate the $r-i$ color from the better measured $g-i$ color using a stellar locus relation ${ }^{32}$

$$
\begin{aligned}
g-r= & 1.39\left\{1-\exp \left[-4.9(r-i)^{3}\right.\right. \\
& \left.\left.-2.45(r-i)^{2}-1.68(r-i)-0.050\right]\right\} .
\end{aligned}
$$

\footnotetext{
30 At the $\sim 0.05$ mag accuracy level, $B-V=0.949(g-r)+0.20$; for more accurate $(<0.01 \mathrm{mag})$ transformations see Ivezić et al. (2007b).

31 Available from http://www.sdss.org/dr6/products/spectra/spectroparameters .html.

32 J08 uses a maximum likelihood projection on the mean stellar locus, which avoids this problem. At the bright end that is relevant here, the two methods produce essentially the same results; we opted for the simpler one.
}

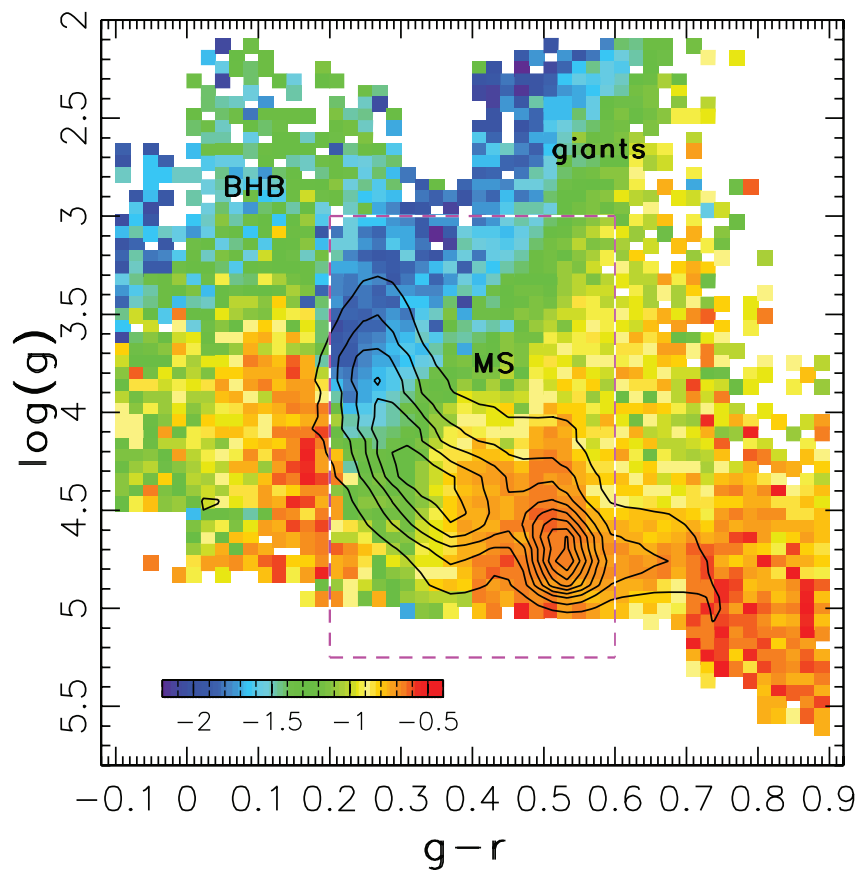

FIG. 1.- Linearly spaced contours showing the distribution of $\sim 110,000$ stars with $g<19.5$ and $-0.1<g-r<0.9$ (corresponding to effective temperatures in the range $4500-8200 \mathrm{~K}$ ) from the SDSS DR6 spectroscopic sample in the $\log g$ vs. $g-r$ plane. The multimodal distribution is a result of the SDSS target selection algorithm. The color scheme shows the median metallicity in all $0.02 \mathrm{mag}$ by 0.06 dex large pixels that contain at least 10 stars. The fraction of stars with $\log g<3$ (giants) is $4 \%$, and they are mostly found in two color regions: $-0.1<$ $g-r<0.2$ (BHB stars) and $0.4<g-r<0.65$ (red giants). They are dominated by low-metallicity stars $([\mathrm{Fe} / \mathrm{H}]<-1)$. The dashed lines outline the mainsequence (MS) region selected for deriving photometric estimates for effective temperature and metallicity.

The selected stars span the 5000-7000 K temperature range (with a median of $5900 \mathrm{~K}$ ), and $99.4 \%$ have metallicity in the range -3 to 0 (with a median of -1.0 ). While the sample is dominated by main-sequence stars (the median $\log g$ is 4.1 , with an rms scatter of 0.44 dex), a small fraction of stars have gravity estimates consistent with giants (see Fig. 1). We exclude $\sim 3 \%$ of stars with $\log g<3$ (which typically have lower metallicity than dwarfs, with a median $[\mathrm{Fe} / \mathrm{H}]=-1.5$; see Fig. 1), resulting in a final calibration sample of 59,789 stars. This fraction of giants is relatively high because the $g-r \sim 0.5$ color range, where the fraction of giants is the highest, was deliberately targeted for SDSS spectroscopy; about $7 \%$ of stars in the subsample with $0.4<g-$ $r<0.6$ have $\log g<3$.

\subsubsection{Effective Temperature}

The dependence of the median effective temperature and metallicity on the position in the $g-r$ versus $u-g$ color-color diagram for the final sample of 59,789 stars is shown in Figure 2. The top left panel demonstrates that the effective temperature, $T_{\text {eff }}$, can be determined from the $g-r$ color alone, with a negligible dependence on the $u-g$ color (the gradient of $\log T_{\text {eff }}$ with respect to the $g-r$ color is at least $\sim 60$ times as large as the gradient with respect to the $u-g$ color). This difference in gradients is due to a general insensitivity to metallicity of relationships between effective temperature and colors at wavelengths longer than $0.4 \mu \mathrm{m}$ (Sandage \& Smith 1963; Mannery \& Wallerstein 1971). The best-fit expression,

$$
\log \left(T_{\text {eff }} / \mathrm{K}\right)=3.872-0.264(g-r)
$$



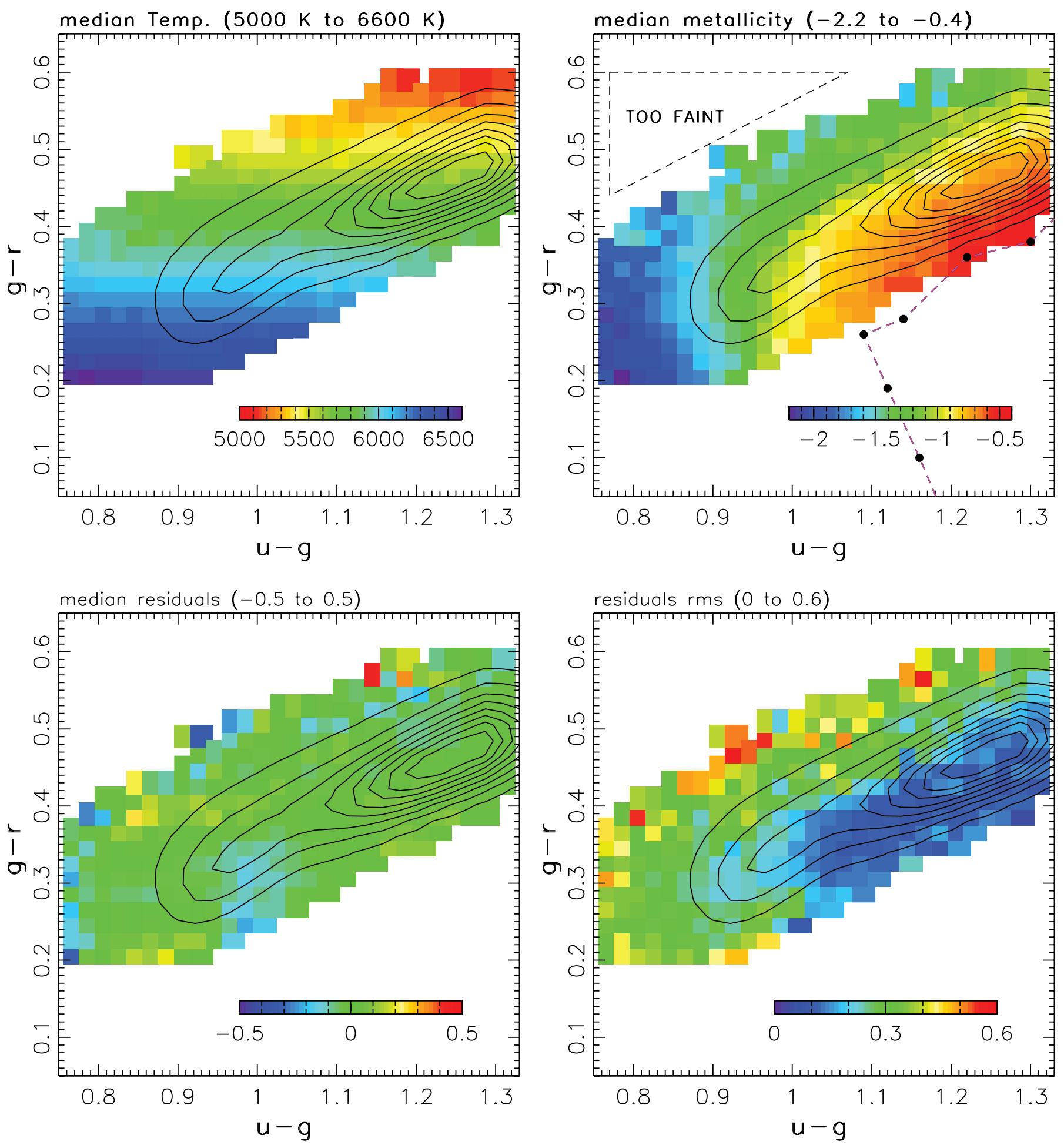

FIG. 2.-Correlation of spectroscopic effective temperature (top left) and metallicity (top right) with the position of a star in the $g-r$ vs. $u-g$ color-color diagram. The color scheme shows the median values in all 0.02 mag by 0.02 mag large pixels that contain at least 10 stars. The distribution of stars in an imaging sample with $g<19.5$ is shown by linearly spaced contours. The filled circles show the synthetic colors for the Pickles (1998) solar metallicity standards ( F0, F2, F5, F6, F8, and G0, from bottom to top), taken from Covey et al. (2007). The triangular region marked "TOO FAINT" in the top right panel contains no stars, due to the $g<19.5$ flux limit and the fact that low-metallicity stars are generally more distant and fainter than high-metallicity stars. The bottom left panel shows the median residuals between the spectroscopic metallicity and photometric estimates based on eq. (4). Their rms scatter (over all pixels) is $0.06 \mathrm{dex}$. The bottom right panel shows a map of the rms scatter of metallicity for individual stars in each pixel. Its median value is $0.21 \mathrm{dex}$. The scatter is larger for weak-lined low-metallicity stars $(\sim 0.3 \mathrm{dex})$ than for high-metallicity stars ( $\sim 0.15$ dex $)$, as expected. 
reproduces SDSS spectroscopic temperature for 59,789 mainsequence stars selected from the $0.2<g-r<0.6$ color range with an rms scatter of 0.007 dex (corresponding to $\sim 100 \mathrm{~K}$ ). When residuals are binned in 0.01 mag wide $g-r$ bins, the largest median residual is $0.003 \mathrm{dex}(\sim 40 \mathrm{~K}$ at the median temperature of $5900 \mathrm{~K}$ ), demonstrating that a linear fit is sufficient. When residuals are binned in 0.1 dex wide metallicity bins, the largest median residual is also 0.003 dex. There is no discernible dependence of residuals on metallicity for stars with $[\mathrm{Fe} / \mathrm{H}]<$ -1 , while for stars with $-1<[\mathrm{Fe} / \mathrm{H}]<-0.5$ a gradient of $\log T$ of 0.008 per decade of metallicity $\left(\operatorname{dex} \operatorname{dex}^{-1}\right)$ is present.

This behavior is consistent with results based on temperatures derived with the infrared flux method (Ramírez \& Meléndez 2005; Casagrande et al. 2006, hereafter CPF06). For example, the expression for effective temperature as a function of $B-V$ color and metallicity from CPF06 predicts an effective temperature of $5700 \mathrm{~K}$ for $B-V=0.6(g-r=0.425)$ and metallicity of -1.0 , with the latter corresponding to the median metallicity of stars in the SDSS spectroscopic sample. The effective temperature predicted by equation (3) is $5750 \mathrm{~K}$ (a discrepancy of $0.004 \mathrm{dex}$ ), and the median spectroscopic temperature for stars with $0.42<g-r<0.43$ is $5730 \mathrm{~K}$. We note that both the CPF06 relation and Ramírez \& Meléndez (2005; see Figs. 1 and 10) predict a steeper dependence of effective temperature on metallicity: at $B-V=0.6$ the predicted effective temperature increases by $180 \mathrm{~K}$ as metallicity increases from -1.5 to -0.5 , while in the SDSS spectroscopic sample the corresponding temperature increase is $50 \mathrm{~K}$. Discrepancies with the expression proposed by Sekiguchi \& Fukugita (2000) are somewhat larger. Their effective temperature scale is cooler by $\sim 130 \mathrm{~K}$ than the SDSS scale, and $\log T_{\text {eff }}$ residuals are correlated with metallicity and $\log g$ with gradients of about $0.01 \mathrm{dex} \mathrm{dex}^{-1}$. Further details about the behavior of photometric temperature estimator are discussed in Appendix B.

\subsubsection{Metallicity}

As first suggested by Schwarzschild et al. (1955), the depletion of metals in a stellar atmosphere has a detectable effect on the emergent flux, in particular in the blue region where the density of metallicity absorption lines is highest (Beers \& Christlieb 2005 and references therein). The median metallicity of stars selected from the SDSS spectroscopic sample as a function of the $u-g$ and $g-r$ colors shows a complex behavior that is consistent with expectations: the detailed dependence of the UV excess (i.e., the $u-g$ color) on metallicity varies with effective temperature (i.e., the $g-r$ color). Even when the $g-r$ versus $u-g$ plane is separated by $g-r=0.4$ into two regions suggested by the metallicity map, at least second-order polynomials, or several piecewise linear fits, are required to avoid systematic errors larger than 0.1 dex. In order to do so for the entire map with a single function, we find that third-order terms are required and model the map as

$$
\begin{aligned}
{[\mathrm{Fe} / \mathrm{H}]_{\mathrm{ph}}=} & A+B x+C y+D x y+E x^{2}+F y^{2} \\
& +G x^{2} y+H x y^{2}+I x^{3}+J y^{3},
\end{aligned}
$$

where $x=(u-g)$ for $(g-r) \leq 0.4$ and $x=(u-g)-2(g-r)+$ 0.8 for $(g-r)>0.4$ (this dual definition is required to describe the map with a single set of coefficients, $A-J), y=(g-r)$, and $(A-J)=(-4.37,-8.56,15.5,-39.0,23.5,20.5,12.1,7.33$, $-10.1,-21.4)$. The above expression describes the median metallicity map shown in the top right panel of Figure 2 with an rms scatter of 0.09 dex. This level of systematic calibration errors is negligible compared to random errors per star $(\sim 0.2 \mathrm{dex}$, due to photometric errors), discussed below, and is comparable to systematic errors in the SDSS spectroscopic metallicity estimates. ${ }^{33}$ A map of the median residuals, when fitting the median metallicity map using equation (4), in the $g-r$ versus $u-g$ plane is shown in the bottom left panel of Figure 2. It illustrates that there is no strong correlation between systematic errors in photometric metallicity computed with equation (4) $(\lesssim 0.1 \mathrm{dex})$ and the $u-g$ and $g-r$ colors.

We compute photometric metallicity estimates for all 59,789 stars in the sample using equation (4) and compare these to the spectroscopic metallicity determinations. The rms scatter of metallicity residuals is 0.24 dex (determined from the interquartile range), and the distribution of residuals is only slightly non-Gaussian ( $97 \%$ of the sample is contained within a $\pm 3 \sigma$ range).

The rms scatter of the metallicity residuals depends on both the apparent magnitude and color of the star under consideration. The bottom right panel of Figure 2 illustrates the color dependence: for low-metallicity stars the rms increases to $\lesssim 0.3 \mathrm{dex}$, while it is about 0.15 dex or less for high-metallicity stars. This is expected, due to the weaker spectral lines in low-metallicity stars (e.g., Du et al. 2004; Keller et al. 2007).

The rms scatter of metallicity residuals increases with the $g$-band magnitude from 0.18 dex for $g<17$ to 0.25 dex at $g=$ 18 and 0.45 dex at $g=19.5$. The random metallicity errors are dominated by the errors in the $u$-band magnitudes. The ratio of this scatter to the scatter expected due to photometric errors (which is readily computed from eq. [4]) is 1.7 and is nearly independent of magnitude. The implied random errors in spectroscopic and photometric metallicity estimates are thus comparable and have similar signal-to-noise ratio properties. In particular, we estimate that random errors in spectroscopic metallicity estimates increase from $0.15 \mathrm{dex}$ for $g<17$ to $0.36 \mathrm{dex}$ at $g=$ 19.5 (for comparison, the corresponding values for photometric metallicity estimates are 0.10 and 0.30 dex, respectively). This seemingly surprising result, that the estimated errors for photometric metallicity are smaller than those obtained for spectroscopic metallicity estimates, despite the former being calibrated off the latter, is due to the averaging of many spectroscopic estimates in a given small color-color bin when calibrating photometric metallicity, as well as the fact that the signal for photometric metallicity estimates predominantly comes from wavelengths shorter than $0.4 \mu \mathrm{m}$, while for spectroscopic metallicity estimates are obtained from longer wavelengths.

This error behavior limits the application of photometric metallicity estimates, based on SDSS data, to about $g<19.5$. This limit is essentially set by the precision of the $u$-band photometry $(u<20.5)$. Somewhat coincidentally, ${ }^{34}$ this is about the same limiting depth as for the SDSS spectroscopic sample (the spectroscopic targeting limit for the SEGUE survey is $g<20$ ). Despite this limitation, the photometric metallicity estimator given by equation (4) is a valuable tool because it allows metallicity to be determined for all main-sequence SDSS stars in the $0.2<$ $g-r<0.6$ color range. For example, in SDSS DR6, out of $\sim 5.7$ million point sources from this color range that are brighter than $g=19.5$, SDSS spectra classified as stars are available only

\footnotetext{
33 The systematic errors are much larger for stars with $\log g<3$ : for example, for stars with $0.4<g-r<0.6$ and $\log g=2.5$, the photometric metallicity estimate is 0.5 dex larger than the spectroscopic metallicity (when the systematic error vs. $\log g$ trend of about $0.35 \mathrm{dex} \mathrm{dex}^{-1}$ is corrected for, the rms scatter of the metallicity residuals for $\log g<3.5$ is $\sim 0.3$ dex).

34 The similar depths are not entirely independent, as they both reflect the atmospheric and sky properties and various scientific trade-offs, but this discussion is beyond the scope of this paper.
} 
TABLE 1

Sample Distance Limits

\begin{tabular}{|c|c|c|c|c|c|}
\hline$(g-r)$ & $(r-i)^{\mathrm{a}}$ & $M_{g}^{\mathrm{b}}$ & $\begin{array}{c}D^{\mathrm{c}} \\
(\mathrm{kpc})\end{array}$ & $(B-V)^{\mathrm{d}}$ & $M_{V}^{\mathrm{e}}$ \\
\hline 0.2. & 0.03 & 3.25 & 17.8 & 0.35 & 3.11 \\
\hline ................ & 0.08 & 4.55 & 9.8 & 0.46 & 4.36 \\
\hline ................. & 0.12 & 5.48 & 6.4 & 0.57 & 5.25 \\
\hline $0.5 \ldots \ldots \ldots \ldots \ldots \ldots \ldots$ & 0.16 & 6.20 & 4.6 & 0.68 & 5.92 \\
\hline $0.6 \ldots \ldots$ & 0.20 & 6.77 & 3.5 & 0.77 & 6.43 \\
\hline ..................... & 0.24 & 7.24 & 2.8 & 0.86 & 6.84 \\
\hline . & 0.28 & 7.64 & 2.4 & 0.95 & 7.19 \\
\hline $0.9 \ldots \ldots \ldots \ldots \ldots \ldots$ & 0.32 & 8.02 & 2.0 & 1.04 & 7.50 \\
\hline $1.0 \ldots \ldots \ldots \ldots \ldots \ldots \ldots$ & 0.37 & 8.40 & 1.7 & 1.12 & 7.83 \\
\hline $1.1 \ldots \ldots \ldots \ldots \ldots \ldots \ldots$ & 0.42 & 8.81 & 1.4 & 1.21 & 8.18 \\
\hline $1.2 \ldots \ldots \ldots \ldots \ldots \ldots \ldots \ldots$ & 0.48 & 9.27 & 1.1 & 1.29 & 8.58 \\
\hline
\end{tabular}

a The mean $r-i$ color on the main stellar locus for the $g-r$ color listed in the first column, evaluated using eq. (2).

b The absolute magnitude in the $g$ band, evaluated for a fiducial $[\mathrm{Fe} / \mathrm{H}]=$ -1.0 using eq. (1).

${ }^{c}$ The distance for a star with $g=19.5$.

d The Johnson $B-V$ color, computed for convenience from SDSS photometry using transformations from Ivezić et al. (2007b).

e The absolute magnitude in the Johnson $V$ band, computed from $M_{g}$.

for $\sim 94,000$ objects. This implies a sample size increase by about factor of 60 when using photometric metallicity estimates. Furthermore, when deeper data are available, the photometric metallicity estimator can be used to study the metallicity distribution in the Galaxy to distances beyond the reach of mainsequence stars in the spectroscopic sample (a small number of giants in the spectroscopic sample, which reach to distances $\sim 100 \mathrm{kpc}$, cannot be easily recognized using photometry alone; however, see Helmi et al. 2003). Further details about the behavior of the photometric metallicity estimator are discussed in Appendix C.

\section{ANALYSIS OF THE STELLAR PHOTOMETRIC METALLICITY ESTIMATES}

We now use the photometric metallicity estimator developed above to study the stellar metallicity distribution as a function of position in the Galaxy and stellar kinematics. We consider stars in a restricted color range, $0.2<g-r<0.4$, because the redder stars $(0.4<g-r<0.6)$ do not extend as far into the halo (due to their smaller luminosities; Table 1). The small color range also minimizes various selection effects that could be larger for a wider color/luminosity range (such as uncertainties in the photometric parallax relation and contamination by giants). As an additional motivation, in this color range metallicity is nearly a function of the $u-g$ color alone (eq. [C2] in Appendix C), which allows a simple assessment of the impact of photometric errors in the $u$ band on derived metallicity. The adopted $0.2<$ $g-r<0.4$ color range spans about $10 \mathrm{MK}$ spectral subtypes (from $\sim$ F5 to $\sim$ G5; Bailer-Jones et al. 1997, 1998). The median absolute magnitude in this color range is $M_{g}=4.6$, with an rms scatter of $0.3 \mathrm{mag}$ and a difference of $\sim 2.2 \mathrm{mag}$ in $M_{g}$ between the blue and red ends (for a fiducial $[\mathrm{Fe} / \mathrm{H}]=-1$, see Table 1).

We consider two photometric catalogs constructed using SDSS data. A catalog of co-added repeated observations (10 on average; Ivezić et al. 2007a), known as the SDSS Stripe 82 catalog, provides improved photometric accuracy to a fainter flux limit in $\sim 300 \mathrm{deg}^{2}$ of sky. For example, while single-epoch SDSS data deliver a median $u-g$ error of 0.06 mag at $g=19.5$ (for point sources with $0.2<g-r<0.4)$, the same level of accuracy is extended to beyond $g=20.5$ in the co-added catalog. This allows us to study the metallicity distribution all the way to the diskhalo interface, at several kiloparsecs from the Galactic plane, with small metallicity errors. At the bright end, the random errors in the $u-g$ color are $0.01 \mathrm{mag}$ in the co-added catalog and $0.025 \mathrm{mag}$ in single-epoch data (an error in the $u-g$ color of 0.02 mag induces a metallicity error in $[\mathrm{Fe} / \mathrm{H}]$ that varies from 0.02 dex at $[\mathrm{Fe} / \mathrm{H}]=-0.5$ to $0.11 \mathrm{dex}$ at $[\mathrm{Fe} / \mathrm{H}]=-1.5$ ). This improvement in photometric metallicity accuracy by more than a factor of 2 enables robust estimates of the metallicity distribution width for disk stars. However, an important disadvantage of using the co-added catalog is its very small sky coverage. Hence, we extend our study to a significant fraction of the Galaxy by using a wide-area catalog based on SDSS DR6. This catalog covers an area $\sim 30$ times larger than the deep co-added catalog, at the expense of $\mathrm{a} \sim 1$ mag shallower sample.

We begin our analysis with a discussion of the stellar distribution in the $g$ versus $u-g$ color-magnitude diagram, which reveals several features that are central to the conclusions of this paper. While this diagram maps well to a distance versus metallicity plane, as discussed in $\S 3.2$, we choose to first describe these features using directly observed quantities. When discussing positions of stars in the Milky Way, we use the usual cylindrical coordinate system $(R, \phi, Z)$ aligned with the Galactic center (assumed to be at a distance of $8.0 \mathrm{kpc}$ ) and with the $Z$-axis toward the north Galactic pole. For projections parallel to the Galactic plane, we follow J08 and use right-handed $X$ - and $Y$-coordinates, with the Sun at $X=8.0 \mathrm{kpc}$ and the positive $Y$-axis pointing toward $l=270^{\circ}$.

\subsection{The Bimodal $u-g$ Distribution of $F / G$ Stars}

We selected 110,363 sources from $\sim 1.01$ million entries in the Stripe 82 co-added catalog ${ }^{35}$ by requiring at least four detections in the $u$ band, $0.20<g-r<0.40$, and $g<20.5$. These sources have $\left|\delta_{\mathrm{J} 2000.0}\right|<1.266^{\circ}$ and right ascension in the range $20^{\mathrm{h}} 34^{\mathrm{m}}$ to $4^{\mathrm{h}} 00^{\mathrm{m}}$. For reference, Galactic coordinates, $(l, b)$, are $(46,-24)$, $(96,-60)$, and $(190,-37)$ for $\alpha_{\mathrm{J} 2000.0}=-50^{\circ}, 0^{\circ}$, and $60^{\circ}$, respectively (at $\delta_{\mathrm{J} 2000.0}=0^{\circ}$ ).

The distribution of these stars in the $g$ versus $u-g$ colormagnitude diagram is shown in the top left panel of Figure 3. Bright red $(g<18, u-g \sim 1.1)$ and faint blue $(g>18, u-g \sim$ $0.9)$ features are clearly discernible and are roughly separated by the $u-g=1$ line (corresponding to $[\mathrm{Fe} / \mathrm{H}] \sim-1.0$ ). The marginal $u-g$ distributions for three $g$ slices are shown in the bottom left panel. They can be approximately described by a sum of two $\sim 0.07$ mag wide Gaussians centered on $u-g=0.90$ and 1.07 , with the number ratio of the blue to red components increasing with magnitude from 1:7 in the blue bin to $20: 1$ in the red bin. The blue and red components correspond to distant metalpoor halo stars and more metal-rich and closer disk stars, respectively, as discussed further below. The width of $0.07 \mathrm{mag}$ is sufficiently larger than the median error in the $u-g$ color $(0.05$ mag at $g=20.5,0.04$ mag at $g=20,0.02$ mag at $g=19$, and 0.01 mag at $g=17.5$ ) to provide a robust measure of the intrinsic width of the $u-g$ distribution.

In addition to an overall blueing of the median $u-g$ color toward the faint end induced by the varying number ratio of the two components, the median $u-g$ color for each component also becomes bluer, as illustrated in Figure 4 and summarized in

\footnotetext{
35 This catalog is publicly available from http://www.sdss.org/dr6/products/ value_added/index.html.
} 

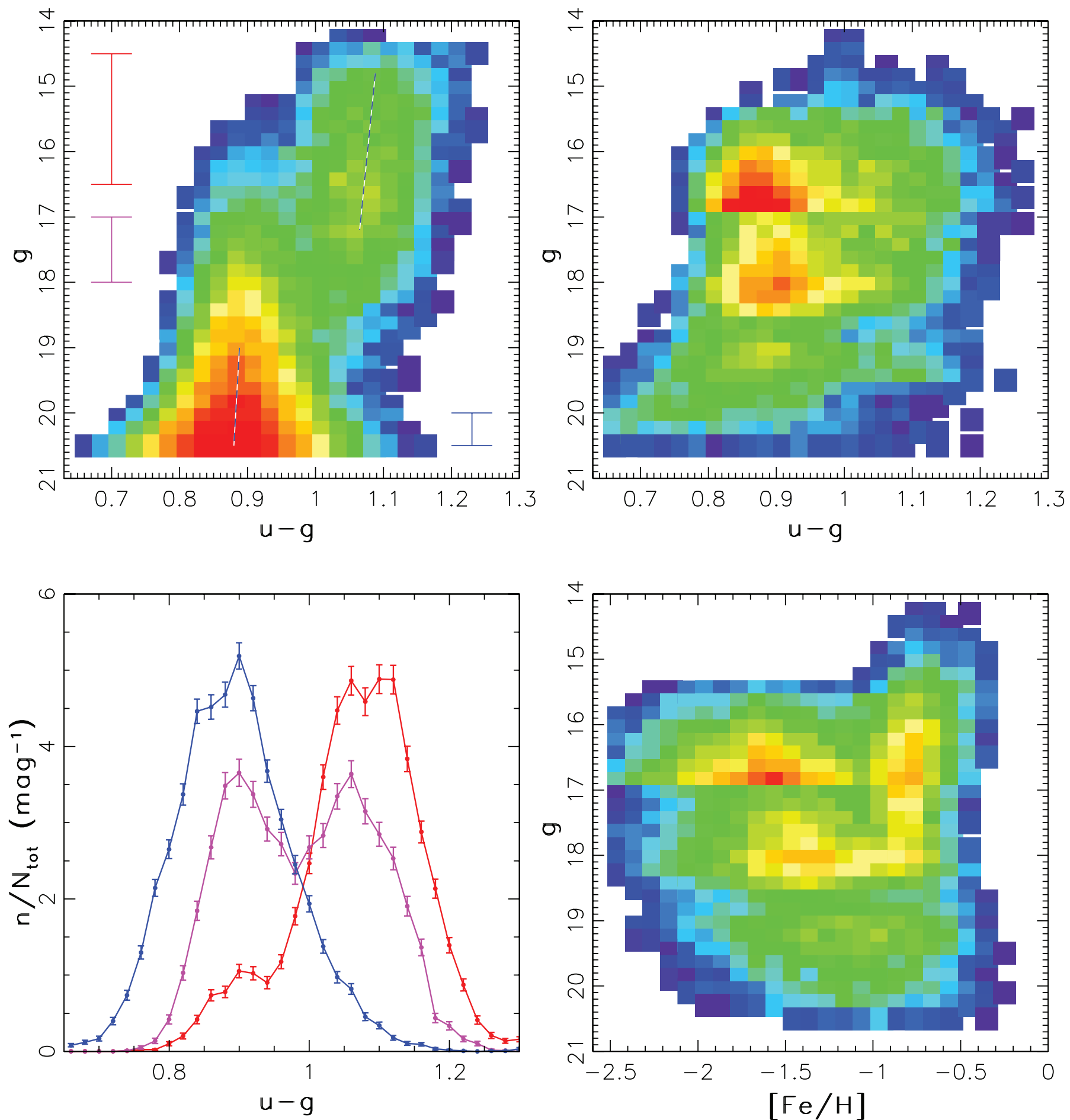

FIG. 3.-Top left panel: Distribution of stars (logarithm of counts in each bin; low to high is blue to green to red) from the SDSS Stripe 82 catalog with $0.2<g-r<$ 0.4 in the $g$ vs. $u-g$ color-magnitude diagram. In this $g-r$ range, the $u-g$ color is a proxy for metallicity (see eq. [C2] in Appendix C). The two concentrations of stars correspond to disk $(u-g \sim 1.1)$ and halo $(u-g \sim 0.9)$ stars, with the dashed lines indicating the change of the median $u-g$ color with magnitude for each concentration. The $u-g$ color distributions in three magnitude slices, marked by vertical bars in the top left panel, are shown in the bottom left panel. All three histograms can be approximately described by a sum of two $\sim 0.07$ mag wide Gaussians centered on $u-g=0.90$ and 1.08 , with the number ratio of blue to red components increasing with magnitude from 1:7 to 20:1. For detailed fits to the $u-g$ color distribution as a function of magnitude, see Fig. 4. The top right panel is analogous to the top left panel, except that a complete imaging sample of stars is replaced by stars from the SDSS spectroscopic survey. The spectroscopic sample is highly incomplete, as evident from the patchy distribution. The bottom right panel shows the same sample of stars from the spectroscopic survey, with the $u-g$ color replaced by spectroscopic metallicity. 

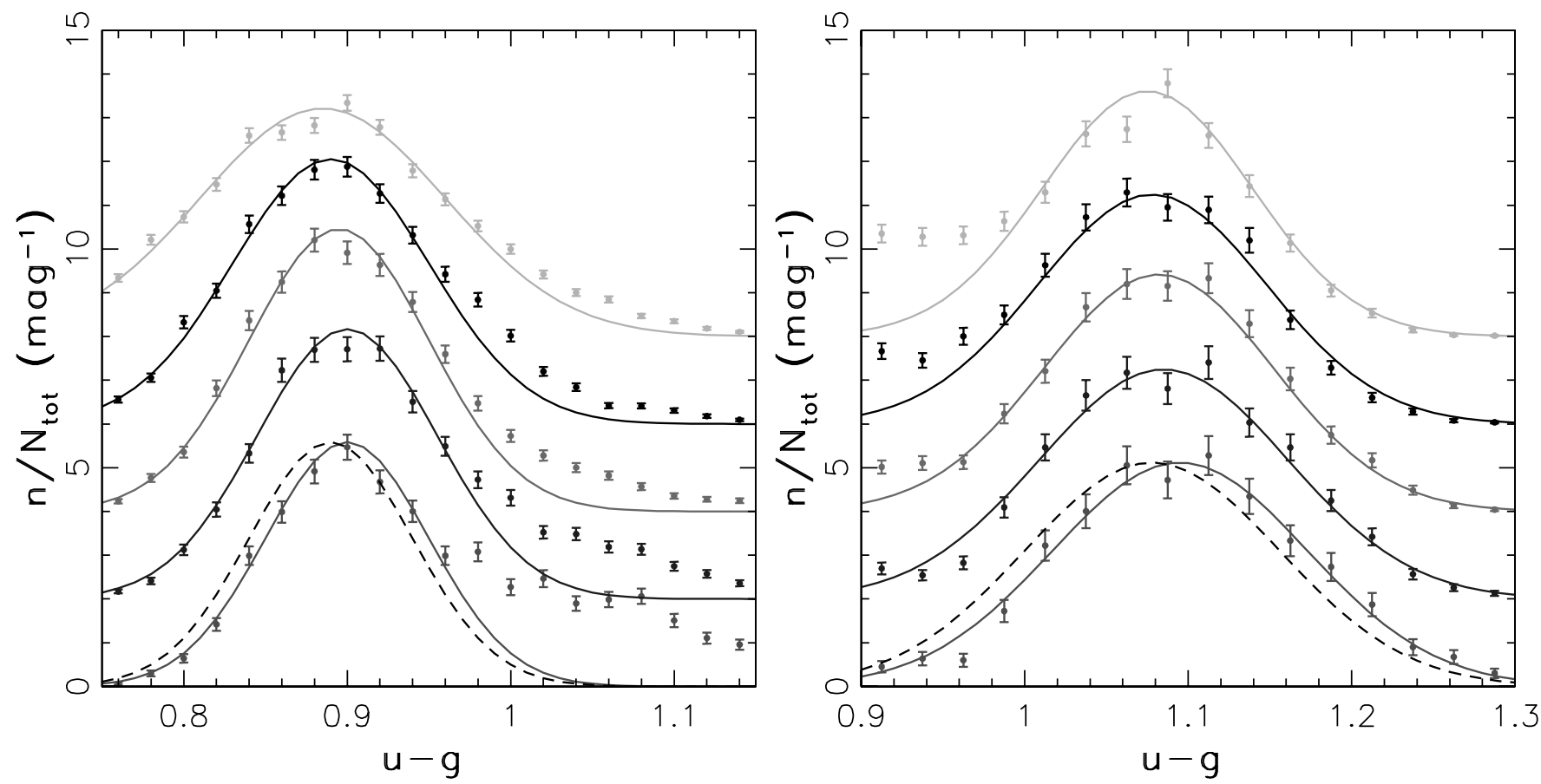

FIG. 4. - Symbols with error bars show the $u-g$ color distributions in 0.5 mag wide magnitude slices (see the top left panel of Fig. 3), in the range $g=18-20.5$ (left) and $g=14.5-17.0$ (right). The bottom histograms correspond to the brightest bin, and each histogram is successively offset by 2 for clarity. The solid lines show the best-fit Gaussians, fitted to data with $u-g<0.95$ (left) and $u-g>1.0$ (right), to minimize contamination by disk and halo stars, respectively. The best-fit parameters are listed in Table 2. The dashed black lines are the same as the solid black lines and are added to illustrate the shift of histograms toward bluer colors for the faint bins. The gradients of the $u-g$ color with respect to the $g$ magnitude are $-0.006 \pm 0.002 \mathrm{mag} \mathrm{mag}^{-1}$ (left) and $-0.012 \pm 0.002 \mathrm{mag} \mathrm{mag}^{-1}$ (right). [See the electronic edition of the Journal for a color version of this figure.]

Table 2. We measure gradients of $-0.012 \mathrm{mag} \mathrm{mag}^{-1}$ for the disk component ${ }^{36}(14.5<g<17)$ and $-0.006 \mathrm{mag} \mathrm{mag}^{-1}$ for the halo component $(18<g<20.5)$, with statistical errors of $\sim 0.002 \mathrm{mag} \mathrm{mag}^{-1}$. Using an approximate mapping from magnitude to distance, these color differences could be produced by a gradient of roughly $0.02 \mathrm{mag} \mathrm{kpc}^{-1}$ between $|Z|=1$ and $2.5 \mathrm{kpc}$ for disk stars and $0.003 \mathrm{mag} \mathrm{kpc}^{-1}$ between $|Z|=4$ and $10 \mathrm{kpc}$

${ }^{36}$ This gradient was accounted for in the definition of the $s$ color by Ivezić et al. (2004), but its meaning was not understood at that time.

TABLE 2

Best-Fit Parameters for the Gaussians Shown in Figure 4

\begin{tabular}{|c|c|c|c|c|}
\hline$g$ Range $^{\mathrm{a}}$ & $N^{\mathrm{b}}$ & $\mu^{\mathrm{c}}$ & $\sigma^{\mathrm{d}}$ & $\begin{array}{c}\mu \text { Error }^{\mathrm{e}} \\
(\mathrm{mmag})\end{array}$ \\
\hline $15.0-15.5 \ldots \ldots \ldots \ldots \ldots \ldots \ldots$ & 1087 & 1.095 & 0.078 & 2.4 \\
\hline $15.5-16.0 \ldots \ldots \ldots \ldots \ldots \ldots$ & 1605 & 1.085 & 0.076 & 1.9 \\
\hline $16.0-16.5 \ldots \ldots \ldots \ldots \ldots \ldots \ldots$ & 1911 & 1.082 & 0.070 & 1.6 \\
\hline $16.5-17.0 \ldots \ldots \ldots \ldots \ldots \ldots \ldots$ & 2328 & 1.078 & 0.070 & 1.5 \\
\hline $17.0-17.5 \ldots \ldots \ldots \ldots \ldots \ldots$ & 2590 & 1.075 & 0.064 & 1.3 \\
\hline $18.0-18.5 \ldots \ldots \ldots \ldots \ldots \ldots$ & 3348 & 0.899 & 0.050 & 0.9 \\
\hline $18.5-19.0 \ldots \ldots \ldots \ldots \ldots \ldots$ & 3745 & 0.901 & 0.055 & 0.9 \\
\hline $19.0-19.5$...……….... & 4504 & 0.895 & 0.055 & 0.8 \\
\hline $19.5-20.0 \ldots \ldots \ldots \ldots \ldots \ldots$ & 5893 & 0.891 & 0.060 & 0.8 \\
\hline $20.0-20.5 \ldots \ldots \ldots \ldots \ldots \ldots$ & 8712 & 0.886 & 0.075 & 0.8 \\
\hline
\end{tabular}

${ }^{a}$ The $g$ magnitude range.

${ }^{b}$ The number of stars in the bin.

c The best-fit mean $u-g$ color (only data with $u-g>1.0$ are fitted in the five brightest bins, and data with $u-g<0.95$ in the five faintest bins; see Fig. 4).

d The best-fit distribution width.

${ }^{\mathrm{e}}$ The statistical error in the mean. for halo stars. Hence, the color gradient per kiloparsec is about 7 times larger for disk stars.

The detected color gradient cannot be caused by potential errors in the applied corrections for interstellar extinction (Schlegel et al. 1998). The median value of the $u-g$ reddening correction is only $0.05 \mathrm{mag}$, and even the closest stars (at $500 \mathrm{pc}$ ) are well beyond the $\sim 100 \mathrm{pc}$ thick dust layer (J08). Such a gradient ( $\sim 0.05$ mag between $u=14$ and 19 ) could be caused by a nonlinearity in the $u$-band measurements (based on a comparison with independently measured Stetson standard stars, this effect is ruled out for the $g$-band measurements; Ivezić et al. 2007a). However, a $u$-band nonlinearity at the $0.05 \mathrm{mag}$ level is excluded by in situ measurements of the hardware response curve, and a comparison of reductions of SDSS data using several different pipelines (SExtractor, DAOPhot, and DoPhot; Becker et al. 2007) excludes such a large software error in the SDSS photometric pipeline. We proceed with an assumption that this gradient is not a problem in the SDSS data.

For selected stars with $0.2<g-r<0.4$, the $u-g$ color measures metallicity (see Appendix C), and the observed color scatter and color gradients correspond to the metallicity distribution width and metallicity gradients. Because the imaging sample is defined by a simple flux limit, these measurements are relatively easy to interpret. On the contrary, the SDSS spectroscopic sample has an extremely biased distribution (by design) in the $g$ versus $u-g$ color-magnitude diagram, as illustrated in the right panels of Figure 3; it would not be easy to derive a robust selection function. Using equations (1) and (4), we find that the color gradients measured for the imaging sample roughly correspond to a $\sim 0.06$ dex kpc ${ }^{-1}$ metallicity gradient for disk stars at $|Z| \sim$ $1.5 \mathrm{kpc}$ and a $\sim 0.01$ dex kpc ${ }^{-1}$ metallicity gradient for halo stars in the $|Z|=4-10 \mathrm{kpc}$ range (given the distance and sky coordinates, 
the three-dimensional position in the Galaxy can be trivially computed). In the remainder of this section we remap the $g$ versus $u-g$ color-magnitude diagram to a distance-metallicity diagram, discuss the metallicity distribution as a function of the position in the Galaxy, develop a model that captures the data behavior, and correlate the metallicity with the observed stellar kinematics.

\subsection{The Bimodal Metallicity Distribution of Thick Disk and Halo Stars}

Despite its small area, the Stripe 82 catalog covers a substantial range of $R$ and $|Z|$, as shown in the top left panel of Figure 5. As evident from the dependence of the median metallicity on $R$ and $|Z|$, the $|Z|$ gradient is much larger than the radial gradient (by about a factor of 10). Given this large difference in metallicity gradients, we proceed by making the assumption that the metallicity distribution is a function of the $Z$ coordinate only (in $\S 3.5$, we critically examine and justify this assumption using the DR6 catalog). To further minimize the effect of any radial gradient, we constrain the sample to $\sim 34,000$ stars with $7<R / \mathrm{kpc}<9$.

The top right panel of Figure 5 shows the resulting conditional metallicity probability distribution for a given $Z, p([\mathrm{Fe} / \mathrm{H}] \mid Z)$. This distribution is computed as metallicity histograms in narrow $Z$ slices and normalized by the total number of stars in a given slice. Apart from renormalization and the applied $7<R / \mathrm{kpc}<$ 9 selection, this is essentially an upside-down warped version of the $g$ versus $u-g$ color-magnitude diagram shown in the top left panel of Figure 3 . The bright red and faint blue components from Figure 3 are now readily identifiable as the relatively close metalrich disk component and the more distant metal-poor halo component, respectively.

\subsection{A Simple Model for the Conditional Metallicity Probability Distribution}

As is evident from the $p([\mathrm{Fe} / \mathrm{H}] \mid Z)$ map shown in the top right panel of Figure 5 , the $Z$ gradient of the median metallicity map shown in the top left panel of Figure 5 is due to the varying contributions of a metal-rich disk component and a metal-poor halo component. We first attempt to model the $p([\mathrm{Fe} / \mathrm{H}] \mid Z)$ map using two Gaussian components with a $Z$-dependent ratio of their area (components' number ratio)

$$
p(x=[\mathrm{Fe} / \mathrm{H}] \mid Z)=\left(1-f_{H}\right) G\left(x \mid \mu_{D}, \sigma_{D}\right)+f_{H} G\left(x \mid \mu_{H}, \sigma_{H}\right),
$$

where

$$
G(x \mid \mu, \sigma)=\frac{1}{\sqrt{2 \pi} \sigma} e^{-(x-\mu)^{2} / 2 \sigma^{2}} .
$$

The distribution width for both components can be modeled as spatially invariant, $\sigma_{D}=0.16 \mathrm{dex}$ and $\sigma_{H}=0.30 \mathrm{dex}$, as is the case for the median halo metallicity, $\mu_{H}=-1.46$. The median and dispersion for metallicity distribution of halo stars are in good agreement with previous work (e.g., Ryan \& Norris 1991). Due to the small errors in the $u-g$ color for the co-added data, the contribution of measurement errors to $\sigma_{D}$ and $\sigma_{H}$ is very small: the implied intrinsic widths are 0.16 and 0.29 dex, respectively.
Inspection of the $p([\mathrm{Fe} / \mathrm{H}] \mid Z)$ map suggests that the variation of the median metallicity for the disk component ${ }^{37}$ in the $0.5<$ $|Z| /$ kpc $<5$ range can be described as

$$
\mu_{D}(Z)=\mu_{\infty}+\Delta_{\mu} \exp \left(-|Z| / H_{\mu}\right) \operatorname{dex}
$$

with the best-fit values $H_{\mu}=1.0 \mathrm{kpc}, \mu_{\infty}=-0.78$, and $\Delta_{\mu}=$ 0.35 . The best fit is shown by the curved dashed line in the top right panel of Figure 5. The exponential "height," $H_{\mu}$, is constrained to only about $50 \%$ due to covariances with $\mu_{\infty}$ and $\Delta_{\mu}$ (which are constrained to about $0.05 \mathrm{dex}$ ). The implied median metallicity values agree well with a value of -0.7 obtained by Gilmore \& Wyse (1985) (they did not detect a metallicity gradient).

The best-fit $\mu_{D}(Z)$ given by equation (7) is valid only for $|Z|>500$ pc because of the sample bright limit. Close to the plane, the mean and rms scatter of the metallicity distribution are -0.14 and 0.19 for F/G-type dwarfs (Nordström et al. 2004; Allende Prieto et al. 2004) and -0.12 and 0.18 for K-type giants (Girardi \& Salaris 2001), respectively. Hence, the vertical metallicity gradient close to the plane must be larger than $\sim 0.35 \mathrm{dex} \mathrm{kpc}^{-1}$ implied by the extrapolation of equation (7) (because stars on average become more metal-poor by about $0.5 \operatorname{dex}$ between $Z=$ 0 and $|Z|=1 \mathrm{kpc}$ ).

To set the relative normalization of the two Gaussians, $f_{H}(Z)$, we approximately separate halo and disk components by isolating stars with $[\mathrm{Fe} / \mathrm{H}]<-1.1$ and $[\mathrm{Fe} / \mathrm{H}]>-0.9$, respectively. A good description of the data, shown by symbols in Figure 6, is provided by a best-fit function with three free parameters

$$
f_{H}(Z)=\frac{1}{1+a \exp \left[-(|Z| / b)^{c}\right]},
$$

with $a=70, b=240 \mathrm{pc}$, and $c=0.62$. We discuss this function further in $\S 3.3 .4$.

\subsubsection{The "Metal-weak Thick Disk": A Third Gaussian Component or a Non-Gaussian Distribution?}

The difference between the data and a two-Gaussian model described above is shown in the bottom left panel of Figure 5. As is evident, the overall behavior of the two dominant components is captured, but the residual map reveals a feature that contains intermediate-metallicity stars $([\mathrm{Fe} / \mathrm{H}] \sim-1.0)$ within $\sim 2-3 \mathrm{kpc}$ from the plane. This feature includes about $5 \%$ of stars in the sample and is reminiscent of the so-called metal-weak thick disk (Morrison et al. 1990; Chiba \& Beers 2000; Beers et al. 2002). Indeed, it can be satisfactorily modeled as a third Gaussian component with $\mu=-1.0, \sigma=0.10 \mathrm{dex}$, and a strength of $20 \%$ relative to the metal-rich component, as illustrated by the smooth residual map shown in the bottom right panel of Figure 5.

An alternative to postulating a third Gaussian component for equation (5) is to adopt a skewed metallicity distribution for the disk component whose shape need not vary with the distance from the plane [i.e., replacing $G\left(x \mid \mu_{D}, \sigma_{D}\right)$ with a non-Gaussian distribution]. A skewed shape for the metallicity distribution of local F/G dwarfs was also measured by Gilmore et al. (1995), but with an overall offset of $\sim 0.5$ dex toward higher metallicity, as would be expected compared to our data at $|Z|=1 \mathrm{kpc}$.

\footnotetext{
${ }^{37}$ An obvious question is whether the observed variation of the median metallicity for the disk component simply reflects the varying contributions of thin- and thick-disk stars. This question is addressed in detail in $\S$ 3.4.4.
} 

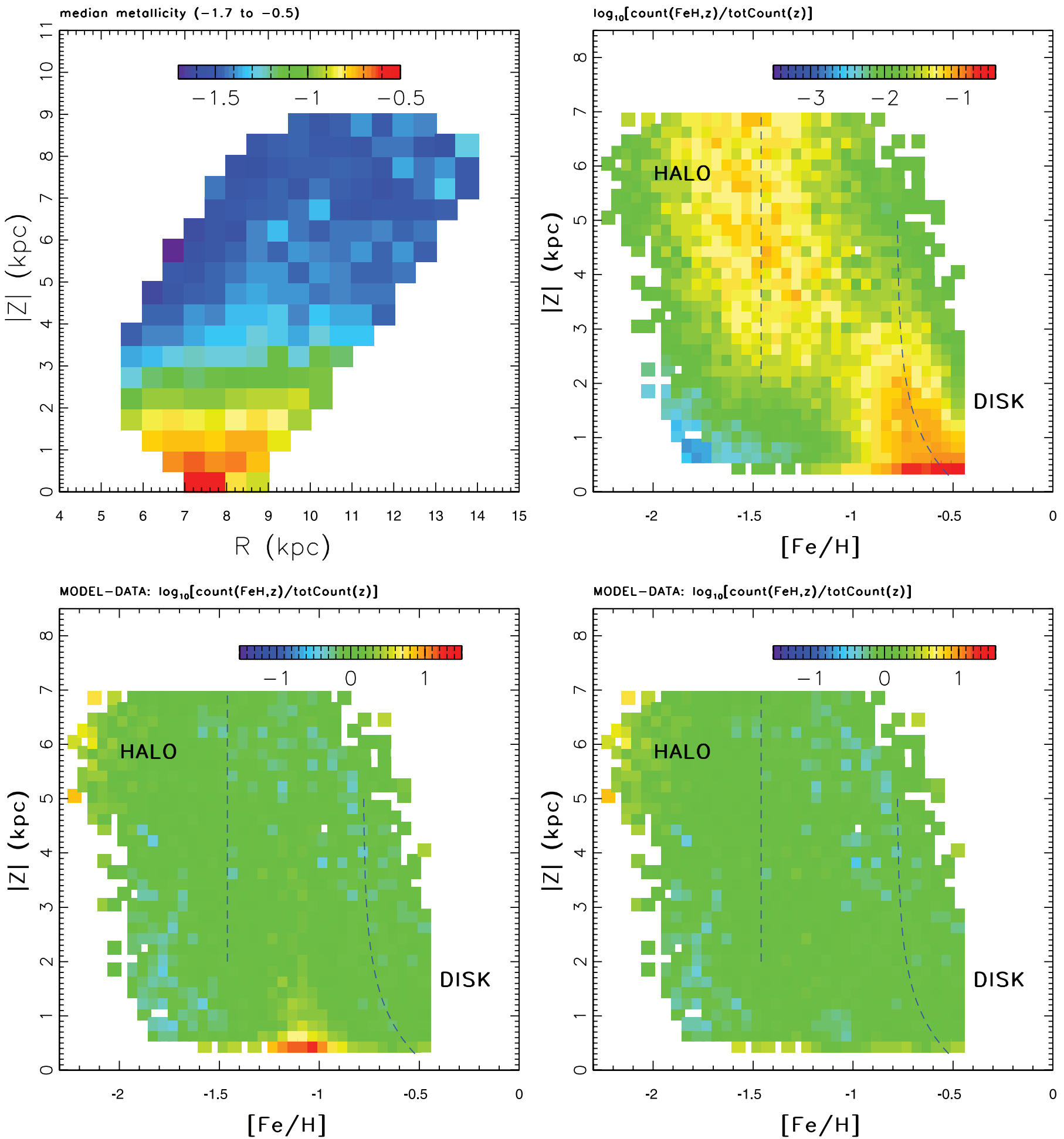

FIG. 5.-Top left panel: Dependence of the median photometric metallicity for $\sim 76,000$ stars from the SDSS Stripe 82 co-added photometric catalog with $14<$ $g<20.5,0.2<g-r<0.4$, and photometric distance in the $0.5-10 \mathrm{kpc}$ range, on cylindrical Galactic coordinates $R$ and $Z$. Note that the $Z$ gradient is much larger than the $R$ gradient $\left(\sim 0.1 \mathrm{dex} \mathrm{kpc}^{-1}\right.$ vs. $\left.<0.01 \mathrm{dex} \mathrm{kpc}-1\right)$. Top right panel: Conditional metallicity probability distribution at a given distance from the Galactic plane for $\sim 34,000$ stars with $0.5<Z / \mathrm{kpc}<7$ and $7<R / \mathrm{kpc}<9$ (the probability density is shown on a logarithmic scale, with its integral normalized to 1 ). The two concentrations of stars correspond to disk $([\mathrm{Fe} / \mathrm{H}] \sim-0.7)$ and halo $([\mathrm{Fe} / \mathrm{H}] \sim-1.5)$ stars. The difference between this map and a two-component Gaussian model described in $\S 3.3$ is shown in the bottom left panel with the same dynamic range for color coding as used in the top right panel. The residual feature visible around $[\mathrm{Fe} / \mathrm{H}] \sim-1.1$ and $Z \sim 1 \mathrm{kpc}$ can be modeled either as a third Gaussian component or by adopting a non-Gaussian metallicity distribution for the disk component. The residuals map for the former is shown in the bottom right panel. 


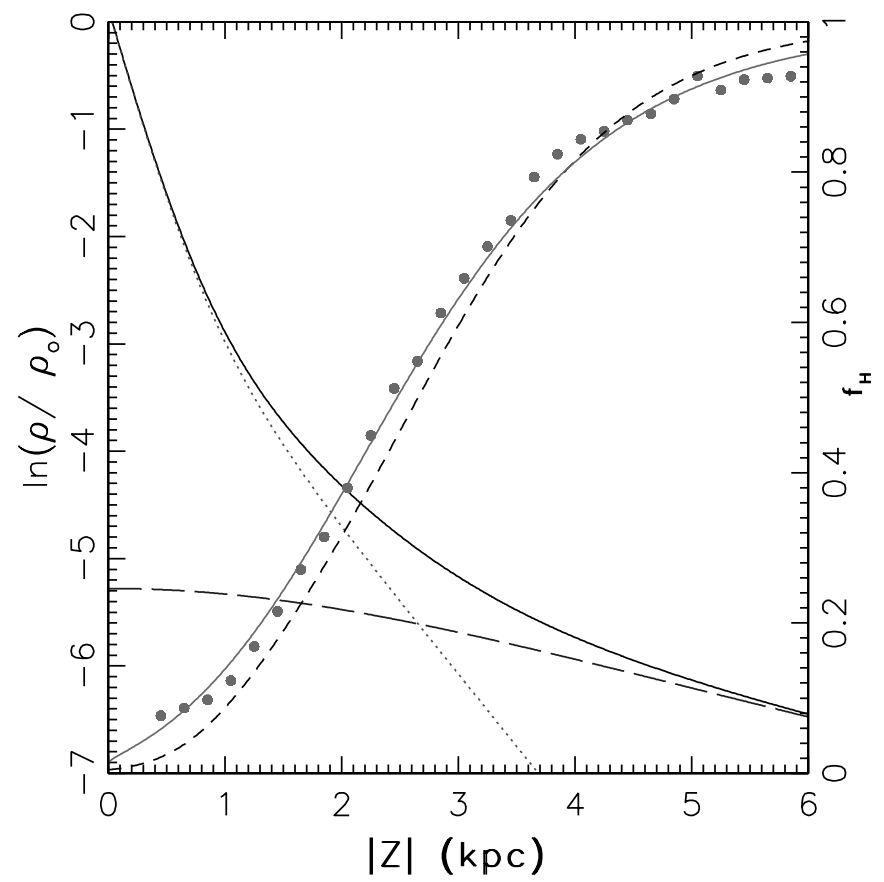

FIG. 6.-Symbols show the number ratio of stars with $[\mathrm{Fe} / \mathrm{H}]<-1$ relative to those with $[\mathrm{Fe} / \mathrm{H}]>-1$, for stars with $7 \mathrm{kpc}<R<9 \mathrm{kpc}$ (with the ratio labeled on the right $y$-axis). Beyond $Z \sim 2.5 \mathrm{kpc}$, metal-poor stars dominate the counts. The solid line passing through the symbols is a three-parameter best fit used in modeling the conditional metallicity probability distribution [equal to 1 / $(1+70 \eta)$, with $\left.\eta=\exp \left(-|Z / 240 \mathrm{pc}|^{0.62}\right)\right]$. The short-dashed line is a prediction for the halo-to-disk counts ratio based on a best-fit Galaxy model to stellar counts from J08. The model includes an oblate power-law halo and exponential thin and thick disks ( $\operatorname{see} \oint 3.3 .4$ ). The disk contribution to the counts is shown by the dotted line [with the $\ln$ (counts) labeled on the left $y$-axis], and the long-dashed line shows the halo contribution. The sum of disk and halo contributions is shown by the solid line. [See the electronic edition of the Journal for a color version of this figure.]

A quantitative validation of such a universal shape for the disk metallicity distribution is shown in Figure 7. In each $Z$ slice that shows evidence for the disk component, the shape of its skewed metallicity distribution can be modeled ${ }^{38}$ as a sum of two Gaussians with fixed positions relative to each other (offset of $0.14 \mathrm{dex}$ ), fixed widths $(0.21 \mathrm{dex}$ for the more metal-poor component, and 0.11 dex for the other), and fixed relative normalization (1.7:1 number ratio in favor of the more metal-poor component). The values of these four parameters were obtained by simultaneously fitting the four histograms shown in Figure 7, with the position of the disk distribution fixed at values computed from equation (7) (the difference between the median given by eq. [7] and the mean for the more metal-poor disk Gaussian is $0.067 \mathrm{dex}$ ). The halo parameter $f_{H}$ was initially estimated using equation (8), while $\mu_{H}=-1.46$ and $\sigma_{H}=0.30$ were kept constant. A few minor adjustments to these three parameters, listed in Table 3, improved the fits. The most notable change is a shift of the halo metallicity distribution by \pm 0.06 dex for $Z>3 \mathrm{kpc}$.

The best-fit values of $f_{H}$ from Table 3 are consistent with equation (8). By retaining that function and adopting the above $Z$-independent two-Gaussian shape description for the disk component, we obtain a residual map that is indistinguishable from that obtained using a third Gaussian component. Hence, we conclude that either hypothesis can explain the data. While both are

\footnotetext{
38 We attempted to fit this skewed distribution using a lognormal distribution, but the detailed shape could not be reproduced.
}

formally based on three Gaussian components, the "universal shape" hypothesis demonstrates that the data do not require the second disk Gaussian to be independent of the first.

It is tempting to identify the two Gaussians that describe the disk component with the thin- and thick-disk contributions. However, the fits presented above are inconsistent with this interpretation. The double-exponential best fit to the observed spatial profile (with scale heights of 245 and $743 \mathrm{pc}$ and a relative normalization of 0.13 ; see $\S 3.3 .4$ and Fig. 6) implies that the ratio of thick- to thin-disk stars should strongly vary from 1.9 in the $Z=$ $0.8-1.2 \mathrm{kpc}$ bin to 14 in the $Z=1.5-2.0 \mathrm{kpc}$ bin, and $>1000$ in the $Z=3.0-4.0 \mathrm{kpc}$ bin. Yet, the metallicity distributions admit a fit based on a constant normalization ratio for the two Gaussian components. Of course, this fitting success alone does not necessarily prove that traditional decomposition into two fixed distributions with a varying normalization ratio is inconsistent with the data. We return to the problem of distinguishing these two hypotheses, which have very different implications for galaxy formation and evolution theories, when discussing correlations with kinematics further below ( $\$ 3.4 .4)$.

\subsubsection{The Effects of Systematic Errors on the Photometric Parallax Relation}

Various systematic errors in the metallicity and distance estimates affect the best-fit models for the metallicity distribution described above. The main sources of systematic errors in the adopted photometric parallax relation are binarity effects (age effects can contribute at most 0.2 mag systematic uncertainty in absolute magnitude at the median color of the sample, $g-r=0.3$, and $\sim 0.1$ mag at $g-r=0.4$; see Appendix A). As discussed in detail by $\mathrm{J} 08$, binarity effects are expected to decrease the inferred distances and disk exponential scale height by about $15 \%$. The impact of binarity on the metallicity determination is hard to estimate without detailed knowledge of both the incidence of binaries and their mass ratio distributions. By performing simulations similar to those described by J08, we find that the worst-case scenario is a system consisting of a luminous low-metallicity primary with $u-g=0.8$ and $g-r=0.2$ and a secondary with $u-g \sim 1.0$ and $g-r=0.5$ (the redder secondaries are too faint to have a larger impact). When such a system is (mis)interpreted as a single star, the distance is underestimated by $15 \%$, the effective temperature is underestimated by $\sim 240 \mathrm{~K}$, and the metallicity is overestimated by $\sim 0.2 \mathrm{dex}$. For components that have mass ratios closer to unity (as suggested by, e.g., Reid et al. 2002), the bias in metallicity will be much smaller, while the bias in distance estimates can increase up to $\sim 40 \%$.

\subsubsection{The Edge of the Thick Disk at $|Z| \sim 5 \mathrm{kpc}$ ?}

Using photographic data for 250 stars, Majewski (1992) advocated an "edge" of the Galactic thick disk at about $5.5 \mathrm{kpc}$ above the Galactic plane. Indeed, the map of conditional metallicity distribution shown in the top right panel of Figure 5 suggests a tantalizing possibility that the metal-rich component does not extend beyond 4-5 kpc from the plane. This visual impression is addressed quantitatively by the histogram shown in the bottom right panel of Figure 7 . The extrapolation of the disk component to the $5-7 \mathrm{kpc}$ bin predicts that the disk should be detectable as a factor of $\sim 2$ excess around $[\mathrm{Fe} / \mathrm{H}] \sim-0.8$, on top of the underlying halo distribution. Such an excess seems consistent with the data and argues against a cutoff in the distribution of disk stars. Due to the small sample size ( $\sim 4800$ stars $)$, the noise is large and the significance of this excess is not overwhelming. Even when the $7<R / \mathrm{kpc}<9$ constraint is removed, the 

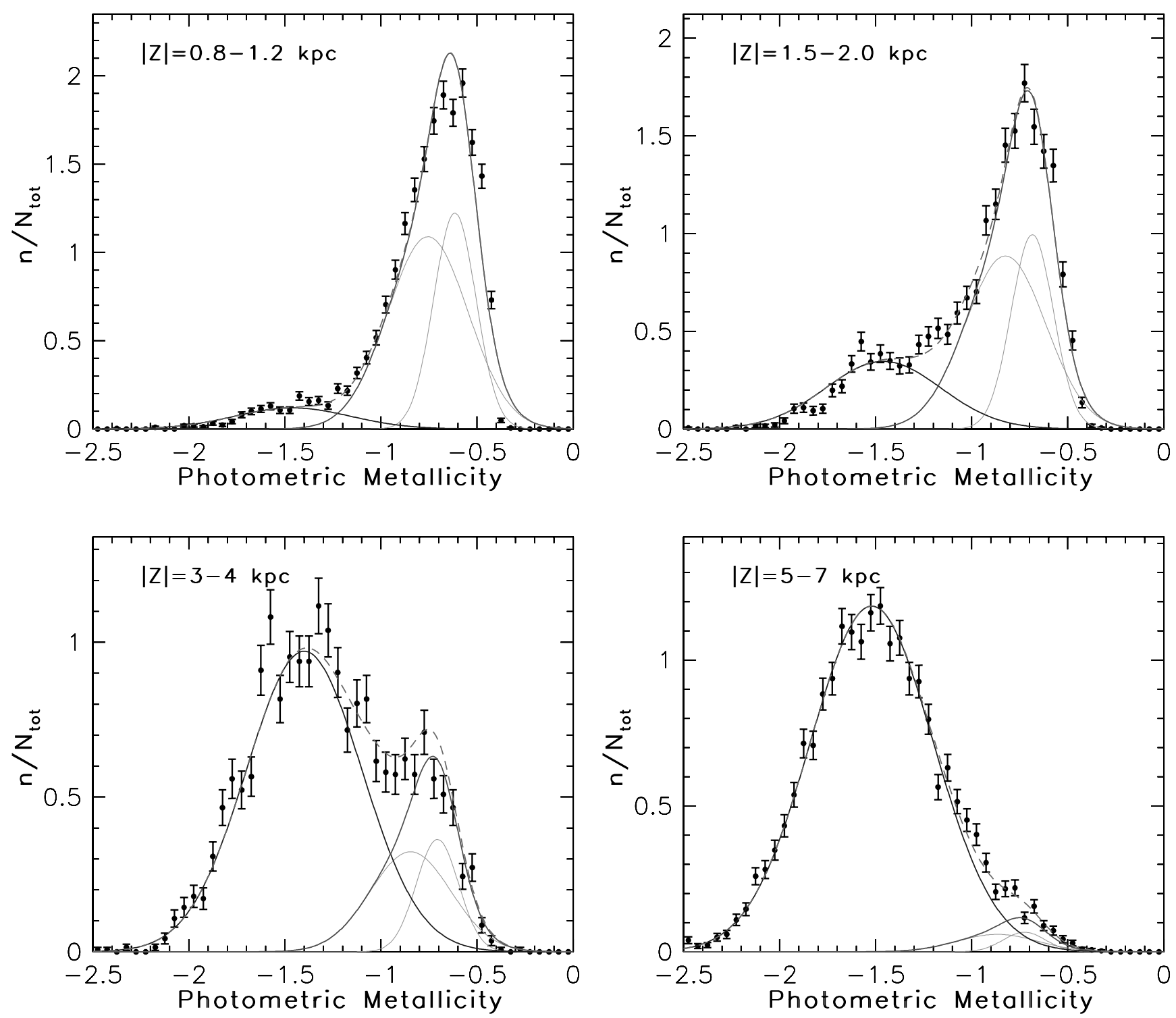

FIG. 7.- Symbols with error bars show the measured photometric metallicity distribution for stars with $0.2<g-r<0.4,7 \mathrm{kpc}<R<9 \mathrm{kpc}$, and distance from the Galactic plane in the range $0.8-1.2 \mathrm{kpc}$ (top left; $\sim 6200 \mathrm{stars}$ ), $1.5-2.0 \mathrm{kpc}$ (top right; $\sim 3800 \mathrm{stars}$ ), $3.0-4.0 \mathrm{kpc}$ (bottom left; $\sim 2800 \mathrm{stars}$ ), and $5.0-7.0 \mathrm{kpc}$ (bottom right; $\sim 6000$ stars). The histograms are essentially horizontal (parallel to $x$-axis) slices at corresponding $|Z|$ intervals through the map shown in the top right panel of Fig. 5 . The dashed lines show a best-fit two-component (halo plus disk) model. The solid lines with a peak at -1.5 show the halo contribution, modeled as a single Gaussian, and the solid lines centered around -0.7 show the contribution of a non-Gaussian disk model, modeled as a sum of two Gaussians shown by the thin solid lines. See $\S 3.3 .1$ and Table 3 for the best-fit parameters. [See the electronic edition of the Journal for a color version of this figure.]

TABLE 3

Best-Fit Parameters for $p([\mathrm{Fe} / \mathrm{H}] \mid Z)$, Shown in Figure 7

\begin{tabular}{|c|c|c|c|c|c|c|c|}
\hline $\begin{array}{l}|Z| \text { Range }^{\mathrm{a}} \\
(\mathrm{kpc})\end{array}$ & $\begin{array}{l}\langle|Z|\rangle^{\mathrm{b}} \\
(\mathrm{kpc})\end{array}$ & $N^{\mathrm{c}}$ & $f_{H}^{\mathrm{d}}$ & $f_{H}^{\mathrm{e}}$ & $f_{H}^{\mathrm{f}}$ & $\mu_{H}{ }^{\mathrm{g}}$ & $\sigma_{H}^{\mathrm{h}}$ \\
\hline $0.8-1.2 \ldots \ldots \ldots+\ldots \ldots \ldots$. & 0.98 & 6,187 & 0.08 & 0.14 & 0.09 & -1.46 & 0.30 \\
\hline $1.5-2.0 \ldots \ldots \ldots \ldots \ldots \ldots$ & 1.72 & 3,842 & 0.24 & 0.30 & 0.26 & -1.46 & 0.30 \\
\hline $3.0-4.0 \ldots \ldots \ldots \ldots \ldots \ldots$ & 3.47 & 2,792 & 0.71 & 0.73 & 0.73 & -1.40 & 0.30 \\
\hline $5.0-7.0 \ldots \ldots \ldots \ldots \ldots \ldots$ & 5.79 & 6,025 & 0.97 & 0.95 & 0.95 & -1.52 & 0.32 \\
\hline
\end{tabular}

${ }^{\text {a }}$ The $|Z|$ range for each bin.

${ }^{\mathrm{b}}$ The median $|Z|$ in each bin

c The number of stars in the corresponding bin.

${ }^{\mathrm{d}}$ The halo-to-disk number ratio predicted by the J08 best-fit model (see $\S 3.3 .4$ ).

e The halo-to-disk number ratio predicted by eq. (8).

${ }^{\mathrm{f}}$ The best-fit halo-to-disk number ratio. The halo parameters are only weakly constrained in the first bin.

g The best-fit mean metallicity for the halo component.

${ }^{\mathrm{h}}$ The best-fit distribution width for the halo component. 
$5<|Z| / \mathrm{kpc}<7$ subsample is still too small ( $\sim 8600$ stars) to significantly overcome counting noise.

We conclude that the Stripe 82 catalog is not sufficiently large to convincingly demonstrate the lack of an edge in the distribution of disk stars. We improve the statistical power of this analysis by using a $\sim 30$ times larger sky coverage provided by the DR6 catalog, as discussed below.

\subsubsection{A Comparison with Results from J08}

The expression given by equation (8) is only a convenient fit that involves a small number of free parameters. The halo-todisk number ratio, $f_{H}$, depends on the disk and halo spatial profiles, both of which are usually modeled using numerous free parameters. As discussed by $\mathrm{J} 08$, a unique solution is not possible without a large sky coverage, and even in such a case it is difficult to obtain. Nevertheless, we can test whether the data for $f_{H}$ discussed here are consistent with the best-fit spatial profiles obtained by $\mathrm{J} 08$, which do not incorporate metallicity information.

J08 show that the stellar number density distribution, $\rho(R, Z, \phi)$, can be well described (apart from local overdensities) as a sum of two cylindrically symmetric components

$$
\rho(R, Z, \phi)=\rho_{D}(R, Z)+\rho_{H}(R, Z) .
$$

The disk component can be modeled as a sum of two exponential disks

$$
\begin{aligned}
\rho_{D}(R, Z)= & \rho_{D}\left(R_{\odot}, 0\right)\left(e^{-\left|Z+Z_{\odot}\right| / H_{1}-\left(R-R_{\odot}\right) / L_{1}}\right. \\
& \left.+\epsilon_{D} e^{-\left|Z+Z_{\odot}\right| / H_{2}-\left(R-R_{\odot}\right) / L_{2}}\right),
\end{aligned}
$$

while the halo component requires an oblate power-law model

$$
\rho_{H}(R, Z)=\rho_{D}\left(R_{\odot}, 0\right) \epsilon_{H}\left[\frac{R_{\odot}^{2}}{R^{2}+\left(Z / q_{H}\right)^{2}}\right]^{n_{H} / 2} .
$$

The best-fit parameters are discussed in detail by J08. We have adopted the following values for parameters relevant in this work (second column of Table 10 from J08): $Z_{\odot}=25 \mathrm{pc}, H_{1}=$ $245 \mathrm{pc}, H_{2}=743 \mathrm{pc}, \epsilon_{D}=0.13, \epsilon_{H}=0.0051, q_{H}=0.64$, and $n_{H}=2.77$.

The fraction of halo stars implied by this model,

$$
f_{H}(R, Z)=\frac{\rho_{H}(R, Z)}{\rho_{D}(R, Z)+\rho_{H}(R, Z)},
$$

agrees reasonably well with $f_{H}$ determined here (see Fig. 6) without any modification. Given that $\mathrm{J} 08$ determined their bestfit parameters using counts at $Z \gtrsim 10 \mathrm{kpc}$ and that the adopted photometric parallax relations are somewhat different, this agreement is remarkable. The short-dashed line representing the J08 model in Figure 6 can be brought into essentially perfect agreement with the data points by decreasing $H_{1}$ and $H_{2}$ by $4 \%$ of their values (i.e., by much less than their uncertainty, $20 \%$, quoted by J08). Alternatively, data points can be moved closer to the J08 model by increasing halo normalization by $24 \%$, to $\epsilon_{H}=0.0063$ $(\sim 1 \sigma$ change). Hence, the results presented here validate the best-fit model from J08.

The best-fit model for stellar counts provides the useful guidance that the counts of thin- and thick-disk stars become equal around $|Z| \sim 1 \mathrm{kpc}$, and that the counts of disk and halo stars be-

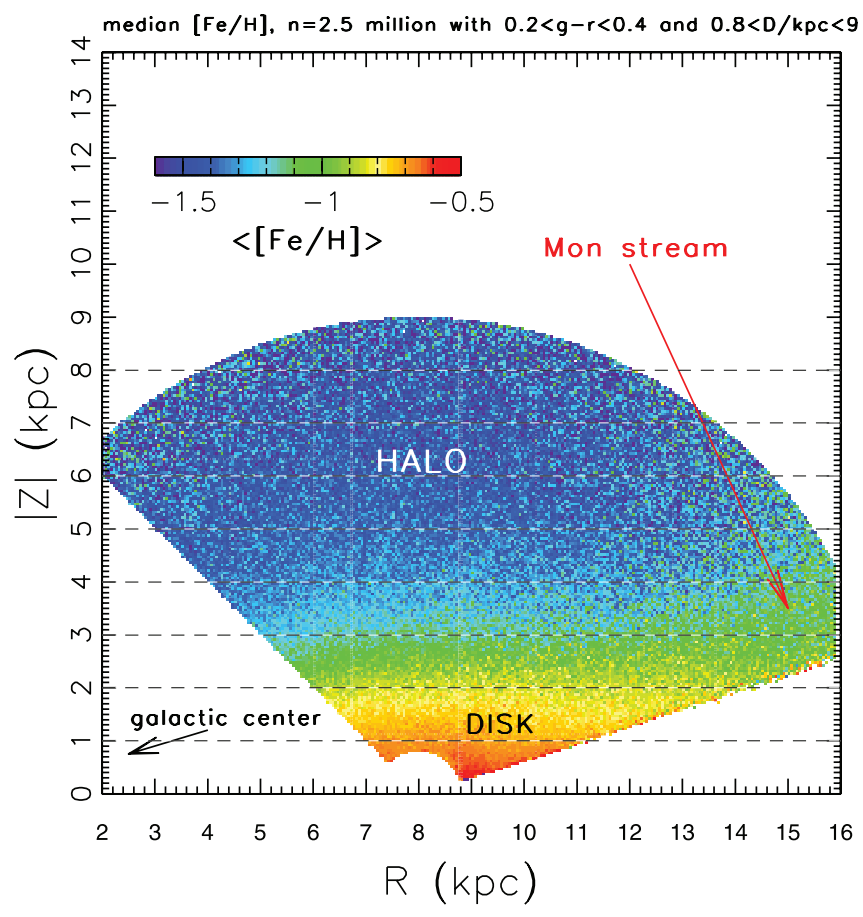

FIG. 8.-Dependence of the median photometric metallicity for $\sim 2.5$ million stars from SDSS DR6 with $14.5<r<20,0.2<g-r<0.4$, and photometric distance in the $0.8-9 \mathrm{kpc}$ range, in cylindrical Galactic coordinates $R$ and $|Z|$ (compare to the top left panel of Fig. 5). There are $\sim 40,000$ pixels (50 pc $\times$ $50 \mathrm{pc}$ ) contained in this map, with a minimum of 5 stars per pixel and a median of 33 stars. Note that the gradient of the median metallicity is essentially parallel to the $|Z|$-axis, except in the Monoceros stream region, as marked.

come equal around $|Z| \sim 2.5 \mathrm{kpc}$. At the bright end of the sample $(500 \mathrm{pc}$ ), thin-disk stars contribute $\sim 70 \%$ of stars to the disk component (with a halo contribution of $\sim 1 \%$ ); at the faint end (5 kpc), halo stars contribute $\sim 90 \%$ of the sample. Hence, the SDSS imaging data are well suited for studying the metallicity distribution all the way from thin disk to halo, through the thickdisk transition, using an essentially complete flux-limited sample of numerous main-sequence stars.

\subsection{Analysis of the Large-Area SDSS Data Release 6 Sample}

SDSS DR6 provides photometry for $\sim 300$ million objects detected over $\sim 10,000 \mathrm{deg}^{2}$ of sky. Using selection criteria listed in $\S 2.3 .1$, and additionally requiring $0.2<g-r<0.4$ and distances in the $0.5-12 \mathrm{kpc}$ range, we selected 2.53 million stars (the extended $0.2<g-r<0.6$ color range includes 5.7 million stars). The significantly increased sky coverage comes at the expense of photometric metallicity precision: for metal-rich disk stars random errors increase from 0.05 dex at a distance of $1 \mathrm{kpc}$ to $0.12 \mathrm{dex}$ at $4 \mathrm{kpc}$, and for metal-poor halo stars they increase from $0.20 \mathrm{dex}$ at $4 \mathrm{kpc}$ to $0.36 \mathrm{dex}$ at $7 \mathrm{kpc}$ (about 3 times as large as for the co-added data discussed above).

The median metallicity map shown in Figure 8 is analogous to that shown in Figure 5 except for the significantly larger sky coverage. The conclusion about the vertical metallicity gradient being much stronger than the radial gradient remains valid. The strong $Z$ gradient is a result of the low-metallicity halo component becoming dominant at $Z$ beyond $\sim 3 \mathrm{kpc}$. The only deviation from this overall trend is seen in the region associated with the Monoceros stream (see $\S 3.5 .1$ for a detailed discussion).

An analogous map constructed using only stars with $[\mathrm{Fe} / \mathrm{H}]>$ -0.9 is shown in the top left panel of Figure 9. While it also displays a much stronger gradient in $Z$ direction, a local maximum 

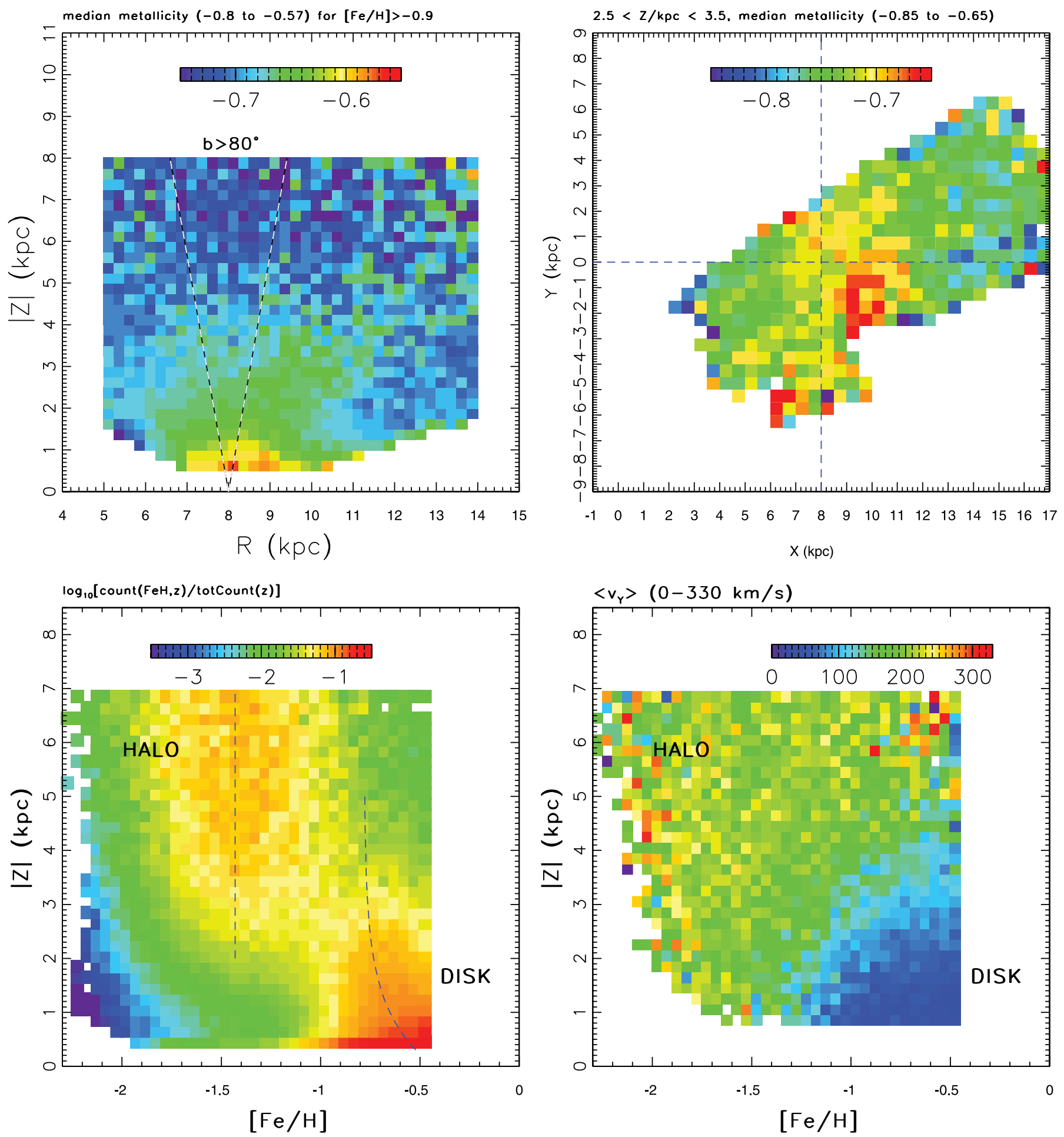

FIG. 9.-Top left panel: Analogous to Fig. 8, except that only 1.1 million stars with $[\mathrm{Fe} / \mathrm{H}]>-0.9$ are used to compute the median $[\mathrm{Fe} / \mathrm{H}]$ (and the display stretch is harder, as indicated in the panel). Note a coherent feature at $R \sim 10 \mathrm{kpc}$ and $Z \sim 3 \mathrm{kpc}$. Its extent parallel to the Galactic plane is shown in the top right panel, which shows the median metallicity for stars with $2.5<Z / \mathrm{kpc}<3.5$ (note the shifted color map). The bottom left panel shows the conditional metallicity probability distribution for $\sim 300,000$ stars from a cylinder perpendicular to the Galactic plane, centered on the Sun, and with a radius of $1 \mathrm{kpc}$. The dashed lines are the same fiducials as shown in the top right panel of Fig. 5 . The bottom right panel shows the median $v_{Y}$ velocity component (heliocentric; the value of $\sim 220 \mathrm{~km} \mathrm{~s}{ }^{-1}$ corresponds to no rotation) as a function of metallicity and distance from the Galactic plane for $\sim 41,000$ stars with $Z<7 \mathrm{kpc}$ and $b>80^{\circ}$ (see the dashed lines in the top left panel). Note the coherent feature with slightly larger $\left\langle v_{Y}\right\rangle$ (by $\sim 20-30 \mathrm{~km} \mathrm{~s}^{-1}$ ) at $[\mathrm{Fe} / \mathrm{H}] \sim-0.6$ and $Z \sim 6.5 \mathrm{kpc}$.

with an amplitude of $\sim 0.1$ dex is discernible at $R \sim 10 \mathrm{kpc}$ and at $\sim 2.5-3.5 \mathrm{kpc}$ above the plane. The $X-Y$ median metallicity map for this $Z$ slice, shown in the top right panel of Figure 9, places this maximum at $X \sim 10 \mathrm{kpc}$ and $Y \sim-2 \mathrm{kpc}$. We address such localized metallicity inhomogeneities further below $(\S 3.5)$.
The conditional metallicity distribution for DR6 stars within a solar cylinder having a radius of $1 \mathrm{kpc}$ is shown in the bottom left panel of Figure 9. As evident, there is a close resemblance between the conditional metallicity distribution map constructed using the Stripe 82 catalog (Fig. 5) and the map based on the DR6 


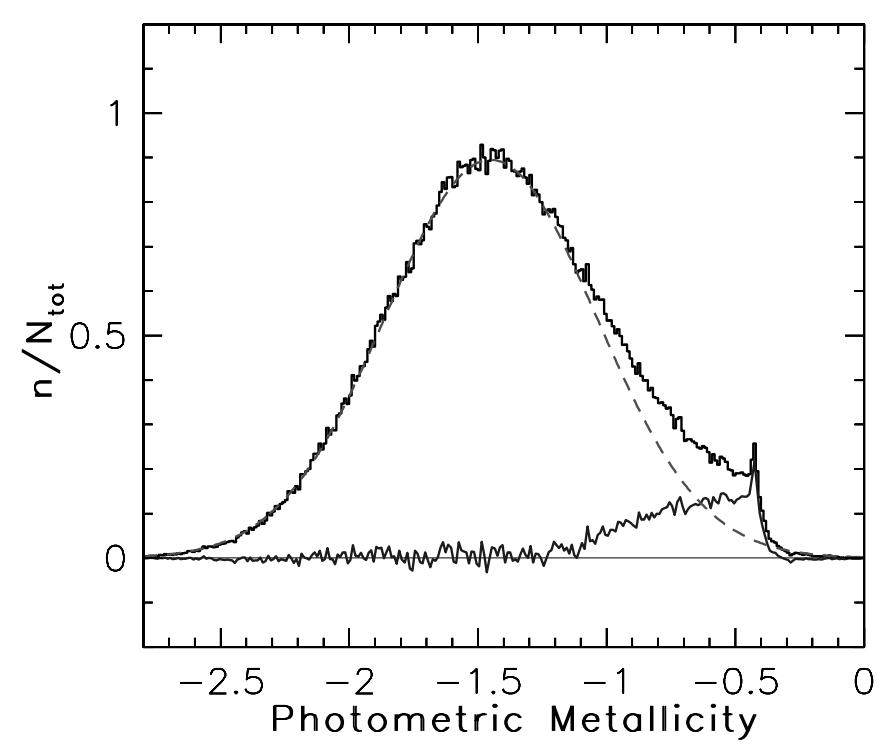

FIG. 10.-Black histogram: Photometric metallicity distribution for $\sim 295,000$ stars with $3<R / \mathrm{kpc}<12$ and $5<|Z| / \mathrm{kpc}<7$ (compare to the bottom right panel of Fig. 7). The dashed line is a Gaussian with a width of $0.41 \mathrm{dex}$ (implying an intrinsic width of $0.32 \mathrm{dex}$ ), centered on $[\mathrm{Fe} / \mathrm{H}]=-1.45$, and an area of 0.92 . The difference between the observed distribution and this Gaussian is shown by the solid gray line and presumably corresponds to disk stars. The small peak around $[\mathrm{Fe} / \mathrm{H}]=-0.5$ is likely an artifact of the photometric metallicity estimation method. [See the electronic edition of the Journal for a color version of this figure.]

catalog. The best-fit parameters listed in Table 3 that describe the histograms shown in Figure 7 are consistent with the DR6 map, when the increased measurement errors are taken into account (disk and halo components are convolved with 0.10 and 0.20 dex wide Gaussians, respectively).

\subsubsection{The Edge of the Thick Disk Revisited}

The large number of stars in the DR6 sample enables a statistically robust analysis of the suspected cutoff in the distribution of thick-disk stars around $|Z| \sim 5 \mathrm{kpc}$. Figure 10 shows the metallicity distribution of $\sim 295,000$ stars with $3<R / \mathrm{kpc}<12$ and $5<Z / \mathrm{kpc}<7$. When a best-fit Gaussian is subtracted from the observed distribution, a highly significant residual at $[\mathrm{Fe} / \mathrm{H}]>$ -1 remains. This residual feature contains about $8 \%$ of the stars ( $\sim 24,000$, so counting noise is not an issue) and suggests that, even so far away from the plane, stars with $[\mathrm{Fe} / \mathrm{H}]>-0.7$ are dominated by disk stars (the disk-to-halo number ratio is about $2: 1)$. The value of $8 \%$ is in excellent agreement with the model discussed in $\S 3.3 .4(8.8 \%$ at $Z=6 \mathrm{kpc})$. An alternative interpretation is that halo metallicity distribution is not Gaussian, but skewed toward high $[\mathrm{Fe} / \mathrm{H}]>-1$ values, with a disk cutoff at $|Z| \lesssim 5 \mathrm{kpc}$. Given the remarkable agreement with a Gaussian for $[\mathrm{Fe} / \mathrm{H}]<-1.1$, it seems more likely that the disk is indeed traceable to beyond $5 \mathrm{kpc}$ from the plane.

The small peak in the observed metallicity distribution visible around $[\mathrm{Fe} / \mathrm{H}]=-0.5$ is probably an artifact of the photometric metallicity estimator. As discussed in $\S \S 2.3 .1$ and 2.3.3, stars with $[\mathrm{Fe} / \mathrm{H}]>-0.5$ may be biased toward somewhat lower metallicity values, which may explain the observed (minor) effect.

\subsubsection{The Metallicity-Kinematics Maps for Stars around the North Galactic Pole}

Kinematic measurements can offer additional insight into Galactic components revealed by the metallicity distribution. The sky coverage of the DR6 catalog includes the anticenter (AC; $l \sim 180^{\circ}$ ) and north Galactic pole (NGP) regions, where propermotion measurements provide a robust constraint on the rotational velocity component even without knowledge of radial velocity. We take proper-motion measurements from the Munn et al. (2004) catalog based on astrometric measurements from SDSS and a collection of Schmidt photographic surveys. The proper-motion measurements publicly available as a part of SDSS DR6 are known to have significant systematic errors (J. Munn et al. 2008, in preparation). Here we use a revised set of propermotion measurements that will become publicly available as a part of SDSS DR7.

Despite the sizable random and systematic astrometric errors in the Schmidt surveys, the combination of a long baseline ( $\sim 50 \mathrm{yr}$ for POSS-I survey) and a recalibration of the photographic data using positions of SDSS galaxies results in median random er-

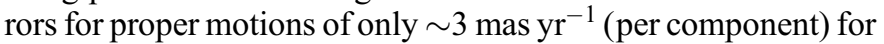
$g<19.5$. Systematic errors are typically an order of magnitude smaller, as robustly determined by B08 using 80,000 spectroscopically confirmed SDSS quasars from Schneider et al. (2007).

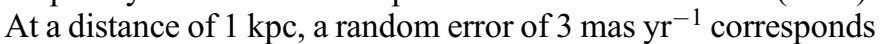
to a velocity error of $\sim 15 \mathrm{~km} \mathrm{~s}^{-1}$, which is comparable to the radial velocity accuracy delivered by the SDSS stellar spectroscopic survey. At a distance of $7 \mathrm{kpc}$, a random error of 3 mas $\mathrm{yr}^{-1}$ corresponds to a velocity error of $100 \mathrm{~km} \mathrm{~s}^{-1}$, which still represents a usable measurement for large samples, given that the systematic errors are much smaller $\left(\sim 10 \mathrm{~km} \mathrm{~s}^{-1}\right.$ at a distance of $7 \mathrm{kpc})$. The faint limit of this catalog $(g \sim 20)$ is well matched to the depth of the SDSS photometric metallicity sample, and thus proper-motion measurements are available for more than $90 \%$ of the $0.2<g-r<0.4$ sample. The kinematics of the SDSS stellar sample, including the mutual consistency of kinematics based on radial velocity and proper-motion measurements, is discussed in detail by B08. Here we briefly present a few results that are directly related to the conclusions of this paper.

For stars toward the Galactic poles, the proper motion measures the radial and rotational velocity components. We select 55,429 unresolved sources closer than $10 \mathrm{kpc}$ with $0.2<g-$ $r<0.4$ and $b>80^{\circ}$ from SDSS DR6. We use the $v_{X}$ and $v_{Y}$ velocity notation to emphasize the spatially constrained nature of this sample and to make a distinction from velocity components computed using a measured radial velocity; in these directions, $v_{X} \sim v_{R}$ and $v_{Y} \sim v_{\Phi}$. We do not correct velocities for the solar motion relative to the local standard of rest (LSR; $v_{X}^{\odot}=$ $-10.0 \pm 0.4 \mathrm{~km} \mathrm{~s}^{-1}, v_{Y}^{\odot}=-5.3 \pm 0.6 \mathrm{~km} \mathrm{~s}^{-1}$, and $v_{Z}^{\odot}=7.2 \pm$ $0.4 \mathrm{~km} \mathrm{~s}^{-1}$; Dehnen \& Binney 1998). Therefore, the mean value of $v_{Y}$ for a nonrotating population is $v_{Y}=-v_{Y}^{\odot}+v_{Y}^{\mathrm{LSR}} \sim 225 \mathrm{~km} \mathrm{~s}^{-1}$, where $v_{Y}^{\mathrm{LSR}} \sim 220 \mathrm{~km} \mathrm{~s}^{-1}$ is the rotational velocity of the LSR (Gunn et al. 1979).

The median heliocentric rotational velocity component as a function of metallicity and distance from the Galactic plane is shown in the bottom right panel of Figure 9. The detailed kinematic behavior shows the same two halo and disk components as implied by the metallicity distribution. The high-metallicity disk component at $[\mathrm{Fe} / \mathrm{H}]>-1$ and $Z<3 \mathrm{kpc}$ lags the local rotation by up to $\lesssim 100 \mathrm{~km} \mathrm{~s}^{-1}$, while the low-metallicity halo component at $[\mathrm{Fe} / \mathrm{H}]<-1$ and $Z>3 \mathrm{kpc}$ has $\left\langle v_{Y}\right\rangle \sim 200 \mathrm{~km} \mathrm{~s}^{-1}$. This strong metallicity-kinematic correlation is qualitatively the same as discussed in the seminal paper by Eggen et al. (1962), except that here it is reproduced in situ with a $\sim 100$ times larger, nearly complete sample, extending it beyond the solar neighborhood.

The variation of the median $v_{Y}$ as a function of distance from the Galactic plane is shown separately for high-metallicity $([\mathrm{Fe} / \mathrm{H}]>$ $-0.9)$ and low-metallicity $([\mathrm{Fe} / \mathrm{H}]<-1.1)$ subsamples in Figure 11. The median $v_{Y}$ for $\sim 40,000$ low-metallicity stars is 

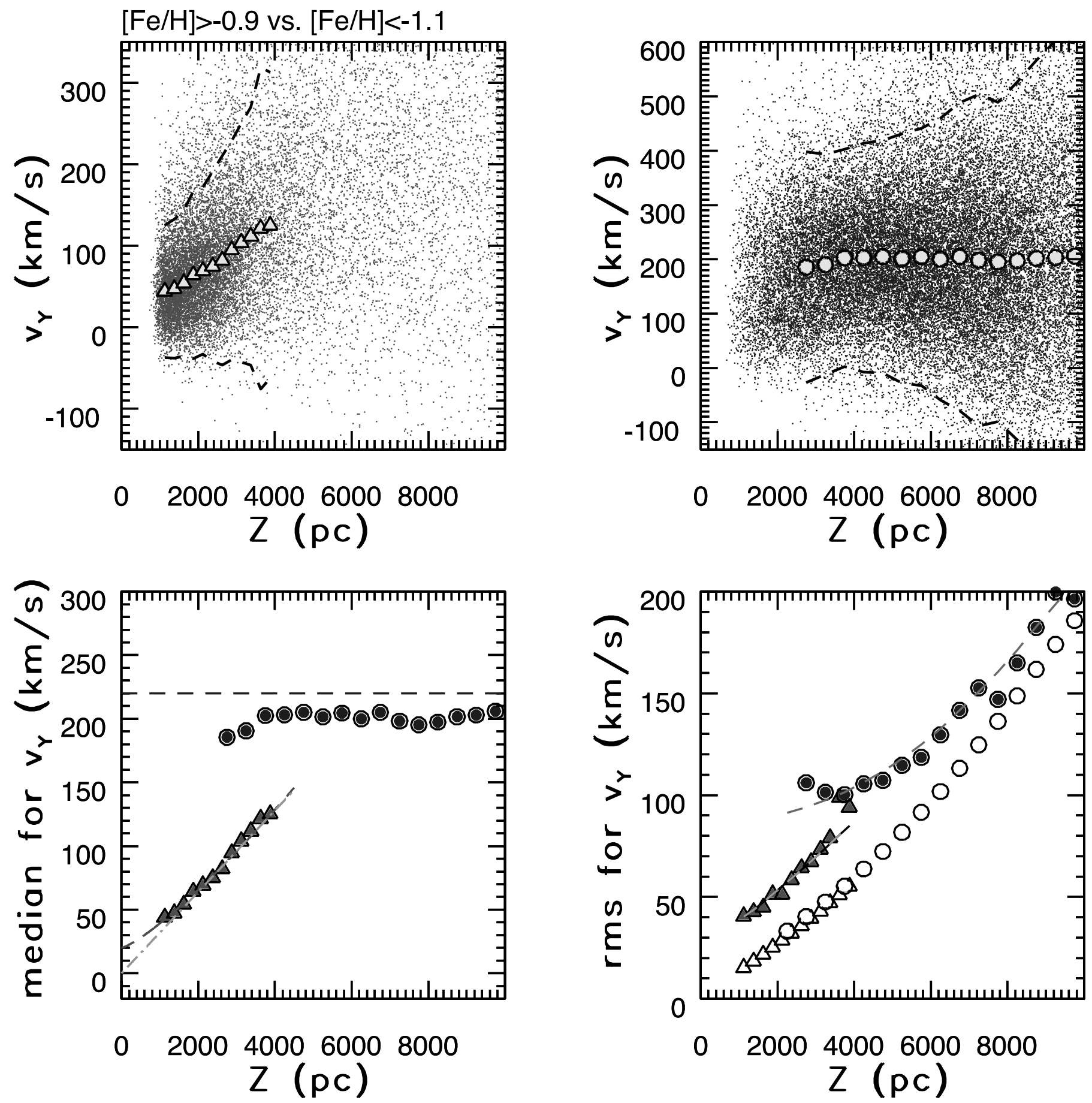

FIG. 11.-Dependence of $v_{Y}$ (heliocentric rotational velocity component) on distance from the plane, $|Z|$, for $\sim 18,000$ high-metallicity $([\mathrm{Fe} / \mathrm{H}]>-0.9$; top left $)$ and $\sim 40,000$ low-metallicity $([\mathrm{Fe} / \mathrm{H}]<-1.1 ;$ top right $)$ stars with $b>80^{\circ}$. In the top two panels individual stars are shown by small dots, and the medians in bins of $Z$ are shown by large symbols. The $2 \sigma$ envelope around the medians is shown by dashed lines. The bottom two panels compare the medians (left) and dispersion (right) for the two subsamples shown in the top panels (filled circles for low-metallicity and triangles for high-metallicity subsamples). The horizontal dashed line at $v_{Y}=220 \mathrm{~km} \mathrm{~s}^{-1}$ in the bottom left panel is added to guide the eye. The other two dashed lines in the bottom left panel are best fits to the observed $v_{Y}(|Z|)$ for high-metallicity stars (see text). The open symbols in the bottom right panel show the median random velocity measurement errors (circles for low-metallicity and triangles for high-metallicity subsamples), and the two dashed lines are best fits to the observed increase of velocity dispersion with $|Z|$. They assume that the intrinsic halo velocity dispersion is constant, $\sigma_{Y}=90 \mathrm{~km} \mathrm{~s}^{-1}$, and that the intrinsic disk velocity dispersion increases as $\sigma_{Y}=26+10|Z| / \mathrm{kpc}\left(\mathrm{km} \mathrm{s}^{-1}\right)$. [See the electronic edition of the Journal for a color version of this figure.]

$205 \mathrm{~km} \mathrm{~s}^{-1}$. The systematic velocity uncertainty, set by the systematic distance and proper-motion errors, is about $10-20 \mathrm{~km} \mathrm{~s}^{-1}$. Therefore, this measurement implies that the halo does not rotate at that level of accuracy, in contradiction with a result based on a similar type of analysis (proper motions and photometric parallax) by Majewski (1992), that the halo counterrotates with a speed of
$-55 \pm 16 \mathrm{~km} \mathrm{~s}^{-1}$ relative to the LSR (based on a sample of a few hundred stars toward the north Galactic pole). In order to make our $v_{Y}$ for halo stars agree with Majewski's result, the distance scale given by the photometric parallax relation needs to be increased by $25 \%$ ( $0.5 \mathrm{mag}$ offset in absolute magnitude scale), which is unlikely (see Appendix A). An alternative explanation 
that proper-motion measurements used here are systematically overestimated by $\sim 2$ mas $\mathrm{yr}^{-1}$ is ruled out by independent data (B08). In addition, the radial velocity measurements from the SDSS spectroscopic survey also indicate no significant halo rotation at the level of $\sim 10 \mathrm{~km} \mathrm{~s}^{-1}$ (Allende Prieto et al. 2006; Ivezić et al. 2006; B08). Recall, as mentioned previously, that our data do not extend very far into the region that Carollo et al. (2007) have argued is dominated by a proposed outer, counterrotating, halo component.

The median $v_{Y}$ for $\sim 18,000$ high-metallicity stars increases with $Z$. We obtained a best fit

$$
\left\langle v_{Y}\right\rangle=20.1+19.2|Z / \mathrm{kpc}|^{1.25} \mathrm{~km} \mathrm{~s}^{-1}
$$

An alternative linear fit

$$
\left\langle v_{Y}\right\rangle=32.2|Z / \mathrm{kpc}| \mathrm{km} \mathrm{s}^{-1}
$$

also provides a good description for $\left\langle v_{Y}\right\rangle$ as a function of $Z$ for stars with $0.2<g-r<0.4$ and $1<Z /$ kpc $<4$. However, B08 show that the detailed kinematics of red stars $(g-r>0.6)$, which sample the smaller $Z$ range ( $<1 \mathrm{kpc}$ ) where the two fits differ by $\sim 20 \mathrm{~km} \mathrm{~s}^{-1}$, prefers the nonlinear form given by equation (13).

The observed velocity dispersion for both halo and disk subsamples increases with $Z$ (see the bottom right panel of Fig. 11). This increase is dominated by increasing measurement errors (mostly due to the fact that even for constant proper-motion errors, the velocity error increases proportionally to distance). We find that the observed velocity dispersion for halo stars can be modeled with a constant intrinsic dispersion of $\sigma_{Y}^{H}=90 \mathrm{~km} \mathrm{~s}^{-1}$. For disk stars, the intrinsic dispersion increases with $Z$ as

$$
\sigma_{Y}^{D}=25.8+10.1|Z / \mathrm{kpc}| \mathrm{km} \mathrm{s}^{-1},
$$

from $\sim 36 \mathrm{~km} \mathrm{~s}^{-1}$ at $Z=1 \mathrm{kpc}$ to $\sim 66 \mathrm{~km} \mathrm{~s}^{-1}$ at $Z=4 \mathrm{kpc}$.

The decrease of rotational velocity with $Z$ (sometimes called velocity lag) was first convincingly detected by Majewski (1992), using photographic data for 250 stars. At $Z \sim 1.5-2.0 \mathrm{kpc}$, he found a lag of $50 \pm 10 \mathrm{~km} \mathrm{~s}^{-1}$, in good agreement with the value of $59 \mathrm{~km} \mathrm{~s}^{-1}$ given by equation (13). Chiba \& Beers (2000) measured a somewhat steeper gradient, of $30 \pm 3 \mathrm{~km} \mathrm{~s}^{-1} \mathrm{kpc}^{-1}$. Using proper-motion data for about a million $\mathrm{M}$ dwarfs, Ivezić et al. (2005) reported essentially the same behavior of rotational velocity at $Z<1 \mathrm{kpc}$, with a median value of $34 \mathrm{~km} \mathrm{~s}^{-1}$ at $Z=$ $1 \mathrm{kpc}$, which agrees well with the lag of $39 \mathrm{~km} \mathrm{~s}^{-1}$ obtained here using F dwarfs. Using SDSS radial velocity data, Allende Prieto et al. (2006) measured a vertical rotational velocity gradient of $16 \mathrm{~km} \mathrm{~s}^{-1}$, which is similar to the results based on proper-motion measurements. Most recently, Girard et al. (2006) used data for 1200 red giants and detected "velocity shear" toward the south Galactic pole. The velocity gradient of $29 \mathrm{~km} \mathrm{~s}^{-1} \mathrm{kpc}^{-1}$ given by equation (13) at $Z=2 \mathrm{kpc}$ is consistent with their value of $30 \pm 3 \mathrm{~km} \mathrm{~s}^{-1} \mathrm{kpc}^{-1}$. They also detected a vertical gradient in the rotational velocity dispersion of $10 \pm 3 \mathrm{~km} \mathrm{~s}^{-1} \mathrm{kpc}^{-1}$, in excellent agreement with the value obtained here. Given the different tracers, analysis methods, and the possibility for north-south asymmetry, the results presented here and those from Girard et al. (2006) are remarkably consistent.

\subsubsection{A Model for the Rotational Velocity Distribution}

The full metallicity/velocity distribution in four $Z$ slices is shown in Figure 12. In agreement with the median behavior shown in Figure 11, close to the Galactic plane the sample is dominated by high-metallicity stars with a small velocity lag and gradually becomes dominated by low-metallicity stars with no net rotation at large $Z$. We find no correlation between velocity and metallicity for distant low-metallicity stars (see the bottom right panel of Fig. 12).

The marginal rotational velocity distributions for maps from Figure 12 are shown in Figure 13. Analogously to modeling the metallicity distributions in $\S 3.3 .1$, we first attempt to fit the dependence of rotational velocity distribution on distance from the Galactic plane, $Z$, using a Gaussian for the halo component and a universal shape, modeled as two coupled Gaussians, for the disk component (an alternative approach based on thin/thickdisk decomposition is described below).

We heuristically model the shape of the disk velocity distribution as a sum of two Gaussians with fixed positions relative to each other (an offset of $34 \mathrm{~km} \mathrm{~s}^{-1}$ ), fixed widths (12 and $34 \mathrm{~km} \mathrm{~s}^{-1}$ ), and fixed relative normalization (3:1 number ratio in favor of the more metal-poor component). Motivated by the behavior of $\left\langle v_{Y}\right\rangle$ for the $[\mathrm{Fe} / \mathrm{H}]>-0.9$ subsample (eq. [13]), we describe the central velocity of the narrower Gaussian as

$$
v_{n}(Z)=-3.0+19.2|Z / \mathrm{kpc}|^{1.25} \mathrm{~km} \mathrm{~s}^{-1}
$$

where the offset of $23 \mathrm{~km} \mathrm{~s}^{-1}$ is due to the difference between the median of the adopted skewed velocity distribution and the mean for the narrower Gaussian component. The values of these four free parameters were obtained by simultaneously fitting the four histograms shown in Figure 13. The halo velocity distribution was kept fixed as a Gaussian centered on $200 \mathrm{~km} \mathrm{~s}^{-1}$ and with a width of $90 \mathrm{~km} \mathrm{~s}^{-1}$.

In each $Z$ bin, the expected velocity measurement error (determined from expected random errors in distance and proper motion) was added in quadrature to the widths of all three Gaussians. For the disk component, we also add in quadrature $10.1 \mid Z / \mathrm{kpc}^{\mathrm{km} \mathrm{s}} \mathrm{km}^{-1}$ based on equation (15). The best-fit parameters are listed in Table 4, and the best fits are shown in Figure 13. As is evident, this simple model of a skewed distribution that slides linearly with $Z$ provides a satisfactory description of the data.

We use the best fits for the marginal metallicity distribution described in $\S 3.3 .1$ (see Fig. 7) and the best fits for marginal rotational velocity distribution discussed above to model the observed joint distributions in the rotational velocity versus metallicity plane, shown in Figure 12. In each $Z$ bin, we simply multiply the best-fit marginal distributions and subtract their product from the observed map. There are two important assumptions underlying this approach: (1) the disk and halo components used to model the two marginal distributions map well onto each other, and (2) the velocity and metallicity distributions of each individual component are uncorrelated.

The above assumptions are borne out by the data. As an illustration of the residual and $\chi^{2}$ maps, we show the $Z=3-4 \mathrm{kpc}$ bin, which contains similar fractions of disk and halo stars (Fig. 14). The observed distribution is successfully modeled to within expected statistical noise ( $\sim 30 \%$ for counts per pixel, on average). The observed lack of a correlation between velocity and metallicity distributions for the disk component is at odds with the traditional thin/thick-disk decomposition, which we address next.

\subsubsection{Difficulties with the Thin-Disk-Thick-Disk Separation?}

Our model fits above show that the data for both the metallicity and rotational velocity distributions of disk stars can be fitted 

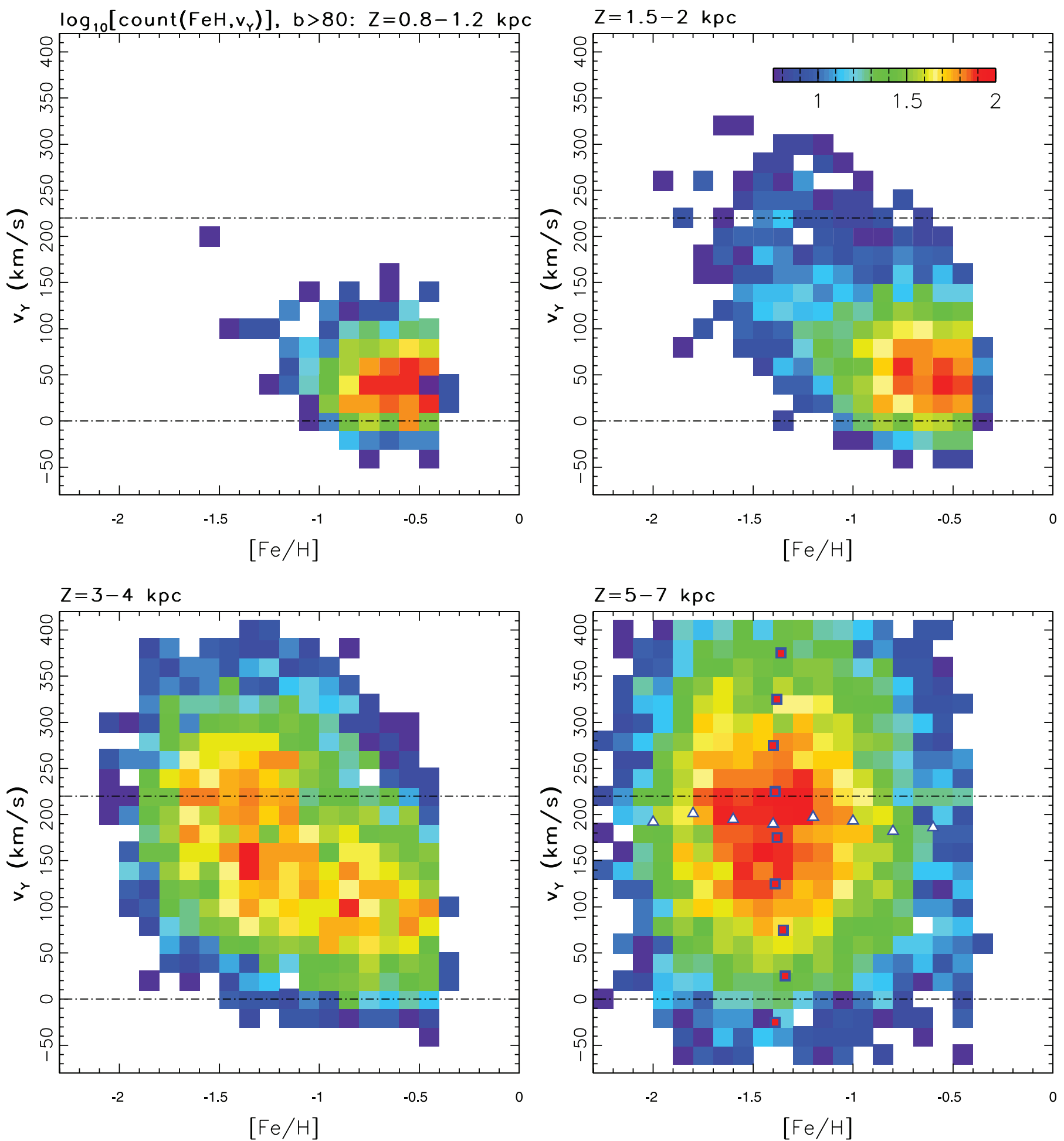

FIG. 12.-Distribution of stars with $0.2<g-r<0.4$ observed toward the north Galactic pole $\left(|b|>80^{\circ}\right)$ in the velocity-metallicity diagrams, and as a function of distance from the plane in the range $0.8-1.2 \mathrm{kpc}$ (top left; $1500 \mathrm{stars}$ ), $1.5-2.0 \mathrm{kpc}$ (top right; $\sim 4100 \mathrm{stars}$ ), 3.0-4.0 kpc (bottom left; $6400 \mathrm{stars}$ ), and $5.0-7.0 \mathrm{kpc}$ (bottom right; $\sim 12,500$ stars). The maps show the logarithm of counts in each pixel, according to the legend shown in the top right panel. Toward the north Galactic pole, the plotted heliocentric velocity component $\left(v_{Y}\right)$ corresponds to the rotational component. Its median value for subsamples selected by $[\mathrm{Fe} / \mathrm{H}]>-1$ is $59 \mathrm{~km} \mathrm{~s} \mathrm{~s}^{-1}$ in the top right panel and $117 \mathrm{~km} \mathrm{~s}^{-1}$ in the bottom left panel. The median value for subsamples selected by $[\mathrm{Fe} / \mathrm{H}]<-1$ is 192 and $203 \mathrm{~km} \mathrm{~s}{ }^{-1}$ in the bottom left and right panels, respectively. For marginal $v_{Y}$ distributions, see Fig. 13. The horizontal lines at $v_{Y}=0$ and $220 \mathrm{~km} \mathrm{~s}^{-1}$ are added to guide the eye. The symbols in the bottom right panel show the median values of metallicity (squares) and $v_{Y}$ (triangles) in narrow bins of the other coordinate. 

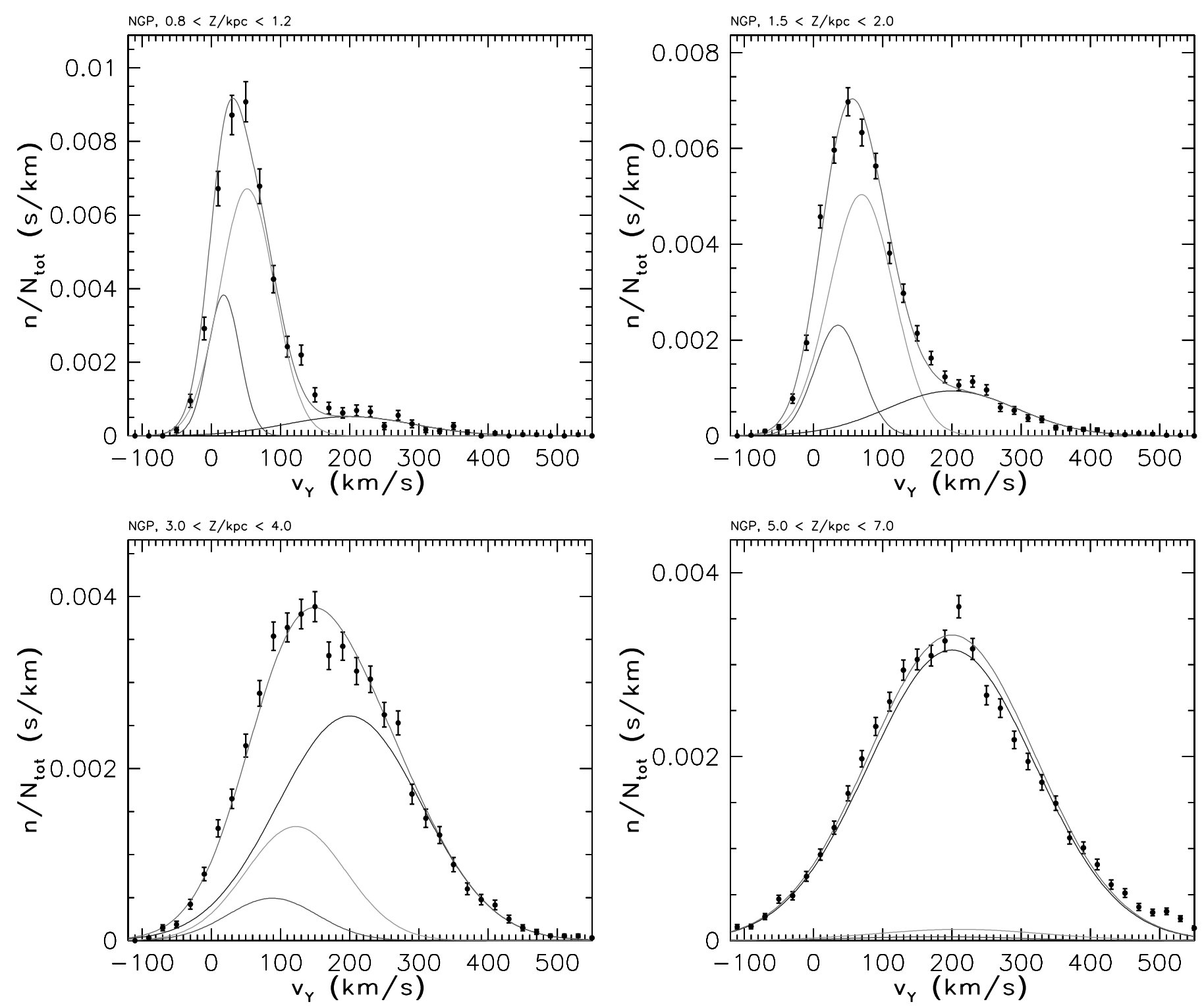

FIG. 13. - Symbols with error bars show the measured rotational velocity distribution for stars with $0.2<g-r<0.4, b>80^{\circ}$, and the distance from the Galactic

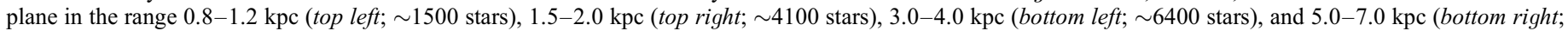
$\sim 12,500$ stars). These histograms are the marginal $v_{Y}$ distributions for the maps shown in Fig. 12. The lines show the contribution of a non-Gaussian disk model (a sum of two Gaussians with fixed, 1:3, relative normalization), halo contribution (a Gaussian), and the highest lines are their sum (see $\S 3.4 .3$ for details and Table 4 for best-fit parameters). [See the electronic edition of the Journal for a color version of this figure.]

TABLE 4

Best-Fit Parameters for $p\left(v_{Y}\right)$, Shown in Figure 13

\begin{tabular}{|c|c|c|c|c|c|c|c|c|c|c|c|}
\hline $\begin{array}{l}|Z| \text { Range }^{\mathrm{a}} \\
(\mathrm{kpc})\end{array}$ & $\begin{array}{l}\langle|Z|\rangle^{\mathrm{b}} \\
(\mathrm{kpc})\end{array}$ & $N^{\mathrm{c}}$ & $\langle g\rangle^{\mathrm{d}}$ & $\begin{array}{c}v_{d 1}{ }^{\mathrm{e}} \\
\left(\mathrm{km} \mathrm{s}^{-1}\right)\end{array}$ & $\begin{array}{c}v_{d 2}{ }^{\mathrm{f}} \\
\left(\mathrm{km} \mathrm{s}^{-1}\right)\end{array}$ & $\begin{array}{c}\sigma_{d 1}{ }^{\mathrm{g}} \\
\left(\mathrm{km} \mathrm{s}^{-1}\right)\end{array}$ & $\begin{array}{c}\sigma_{d 2}{ }^{\mathrm{h}} \\
\left(\mathrm{km} \mathrm{s}^{-1}\right)\end{array}$ & $f_{H}{ }^{\mathrm{i}}$ & $\begin{array}{c}\sigma_{H}{ }^{\mathrm{j}} \\
\left(\mathrm{km} \mathrm{s}^{-1}\right)\end{array}$ & $\begin{array}{c}\sigma_{v}{ }^{\mathrm{k}} \\
\left(\mathrm{km} \mathrm{s}^{-1}\right)\end{array}$ & $\begin{array}{c}\sigma_{v}{ }^{1} \\
\left(\mathrm{~km} \mathrm{~s}^{-1}\right)\end{array}$ \\
\hline $0.8-1.2 \ldots \ldots \ldots \ldots$ & 1.08 & 1526 & 15.2 & 18 & 57 & 23 & 39 & 0.12 & 92 & 14 & 17 \\
\hline $3.0-4.0 \ldots \ldots \ldots \ldots \ldots$ & 3.49 & 6445 & 17.8 & 88 & 122 & 65 & 72 & 0.68 & 104 & 48 & 52 \\
\hline $5.0-7.0 \ldots \ldots \ldots \ldots \ldots \ldots$ & 5.99 & 12452 & 18.9 & 176 & 211 & 118 & 122 & 0.95 & 123 & 93 & 84 \\
\hline
\end{tabular}

${ }^{\text {a }}$ The $|Z|$ range for the bin.

b The median $|Z|$ in the bin.

c The number of stars in the bin.

d The median $g$-band magnitude in the bin.

e The mean velocity for the first Gaussian disk component computed using eq. (16).

${ }^{\mathrm{f}}$ The mean velocity for the second Gaussian disk component, $v_{d 2}=v_{d 1}+34$.

$\mathrm{g}$ The velocity dispersion for the first Gaussian disk component $\left(12 \mathrm{~km} \mathrm{~s}^{-1}\right)$ convolved with measurement errors.

$\mathrm{h}$ The velocity dispersion for the second Gaussian disk component $\left(34 \mathrm{~km} \mathrm{~s}^{-1}\right)$ convolved with measurement errors.

i The best-fit halo-to-disk number ratio.

$\mathrm{j}$ The velocity dispersion for the halo component $\left(90 \mathrm{~km} \mathrm{~s}^{-1}\right)$ convolved with measurement errors.

${ }^{k}$ The median velocity measurement error for stars with $[\mathrm{Fe} / \mathrm{H}]>-0.9$.

${ }^{1}$ The median velocity measurement error for stars with $[\mathrm{Fe} / \mathrm{H}]<-1.1$. 
DATA for $b>80: Z=3-4 \mathrm{kpc}$

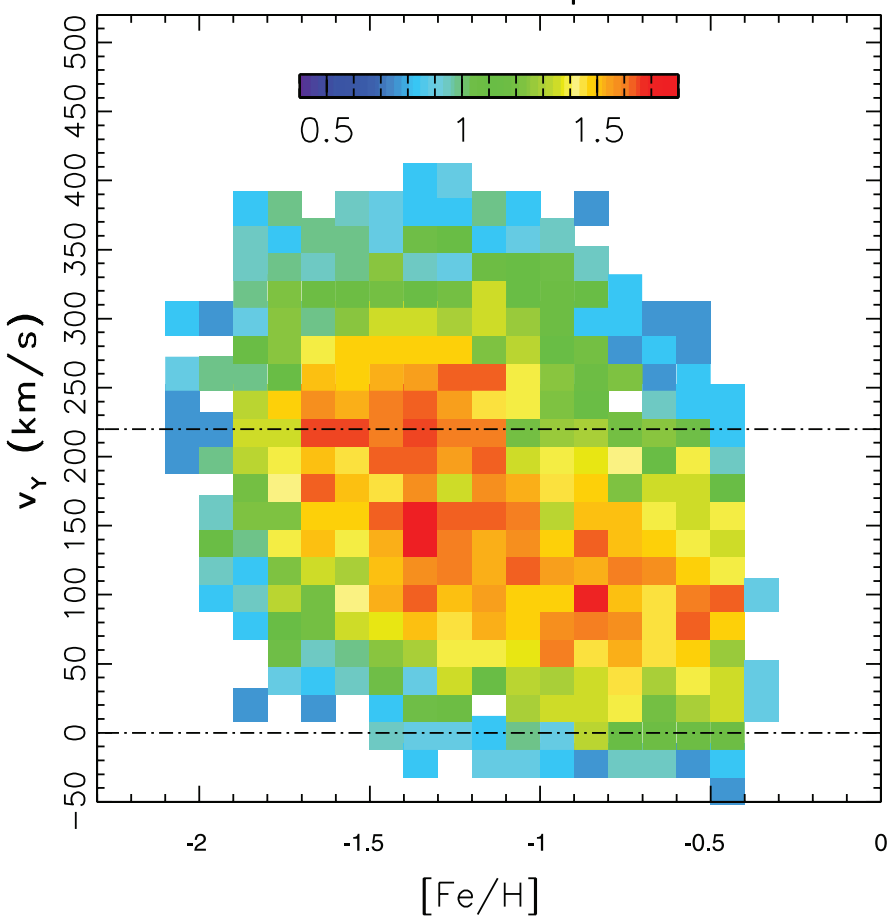

DATA-MODEL for $b>80: Z=3-4 \mathrm{kpc}$

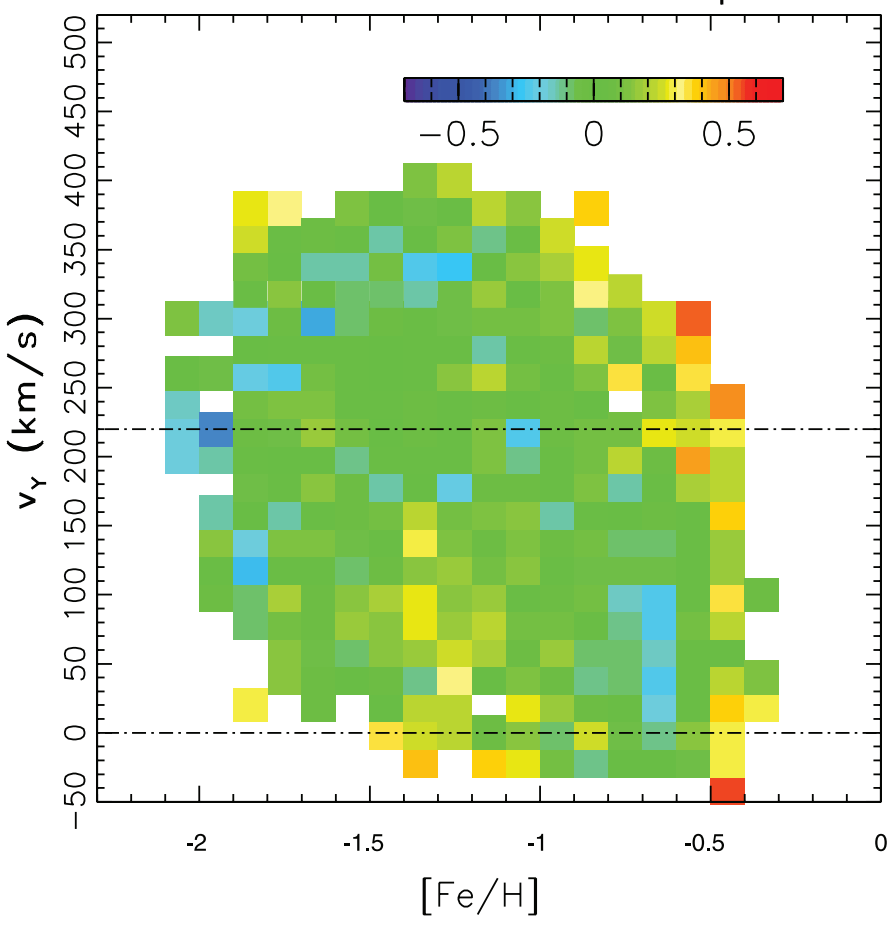

MODEL for $b>80: Z=3-4 \mathrm{kpc}$

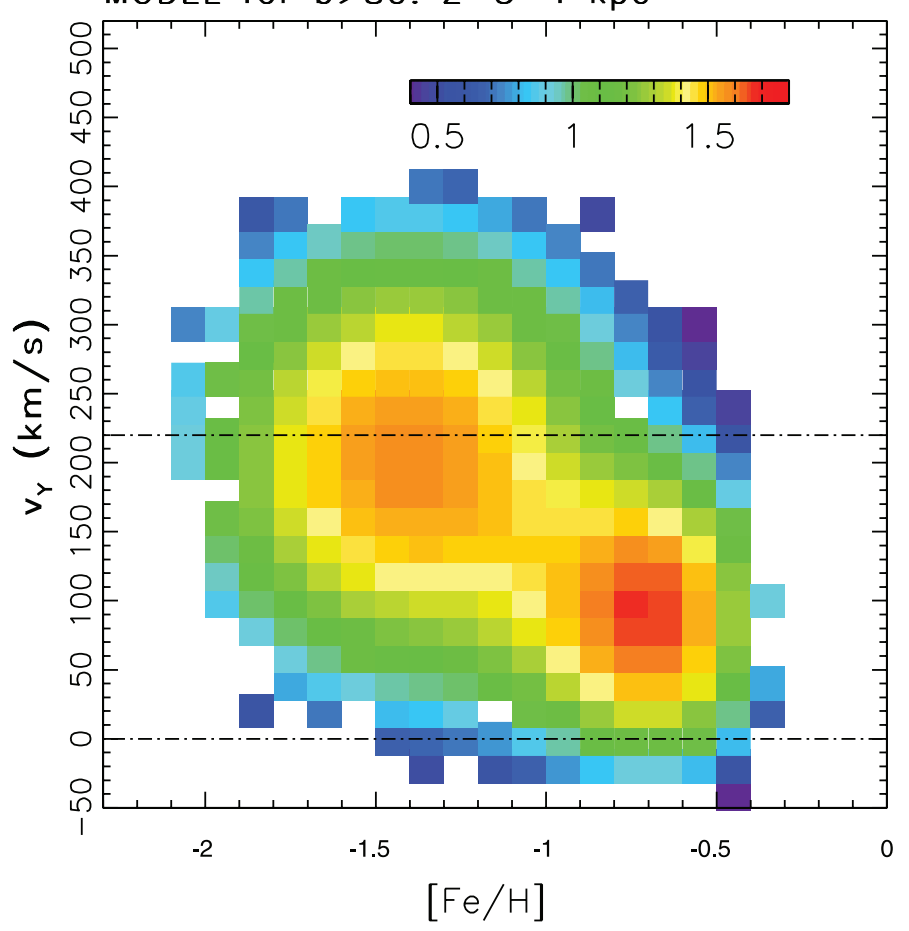

(DATA-MODEL)/NOISE

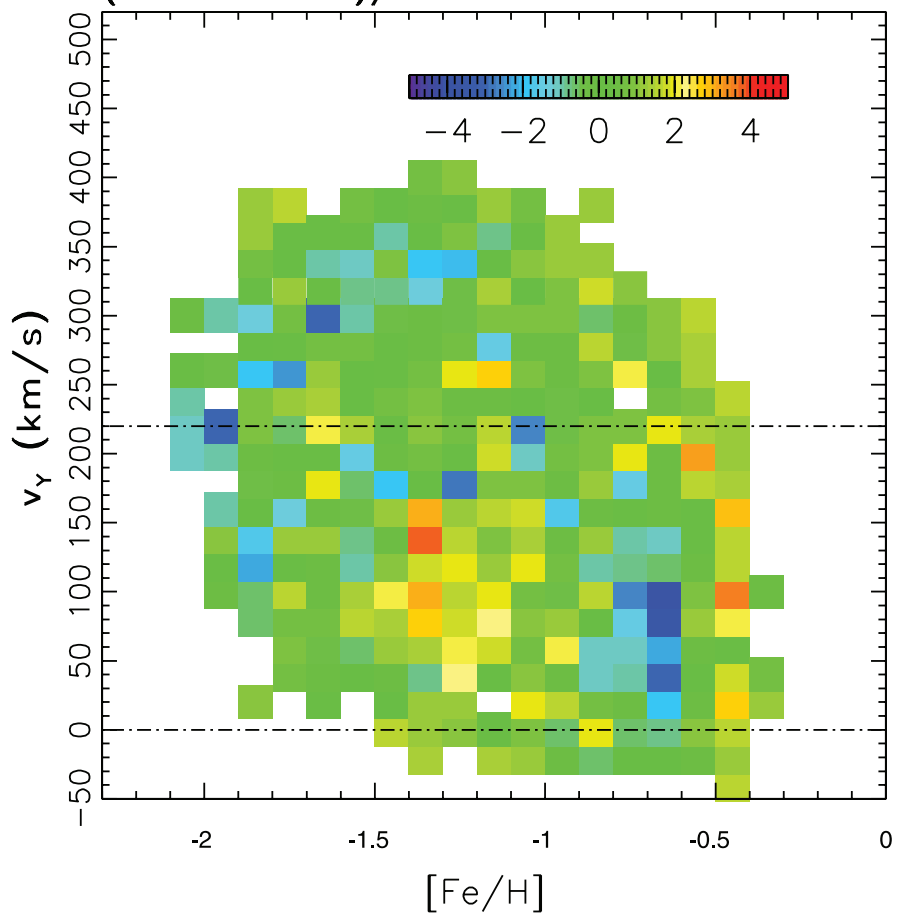

FIG. 14.-Top left panel: Distribution of stars (logarithm of counts in each bin) observed toward the north Galactic pole $\left(|b|>80^{\circ}\right)$, and with $3.0<|Z| / \mathrm{kpc}<4 \mathrm{kpc}$, in the velocity-metallicity diagram (same map as in the bottom left panel of Fig. 12). Top right panel: Best-fit model assuming that velocity and metallicity distributions are uncorrelated, when disk and halo components are treated separately, and are given by the best fits shown in the bottom left panel of Fig. 7 for the metallicity distribution and in the bottom left panel of Fig. 13 for the velocity distribution. Bottom left panel: Logarithm of the data/model ratio, displayed with the same dynamic range as the top two panels. The observed counts are predicted with an rms scatter of $33 \%$. This scatter is consistent with the expected statistical noise. Bottom right panel: Data-model difference map normalized by the expected noise. The rms width of the distribution is 1.09 .

with shapes that do not vary with $Z$. While formally these skewed distributions are modeled as sums of two Gaussians, these Gaussians cannot be readily identified with the classical thin and thick disks. First, their normalization ratio is constant, while double-exponential best fits to the observed spatial profile predict that the fraction of stars in each component should strongly vary with $Z$. Secondly, the normalization ratios are different, 1.7 for the metallicity distributions and 3.0 for the rotational velocity distributions, respectively, which implies that the two components do not perfectly map onto each other.

Despite these difficulties, it is instructive to attempt a traditional thin- plus thick-disk decomposition using the data presented here. 

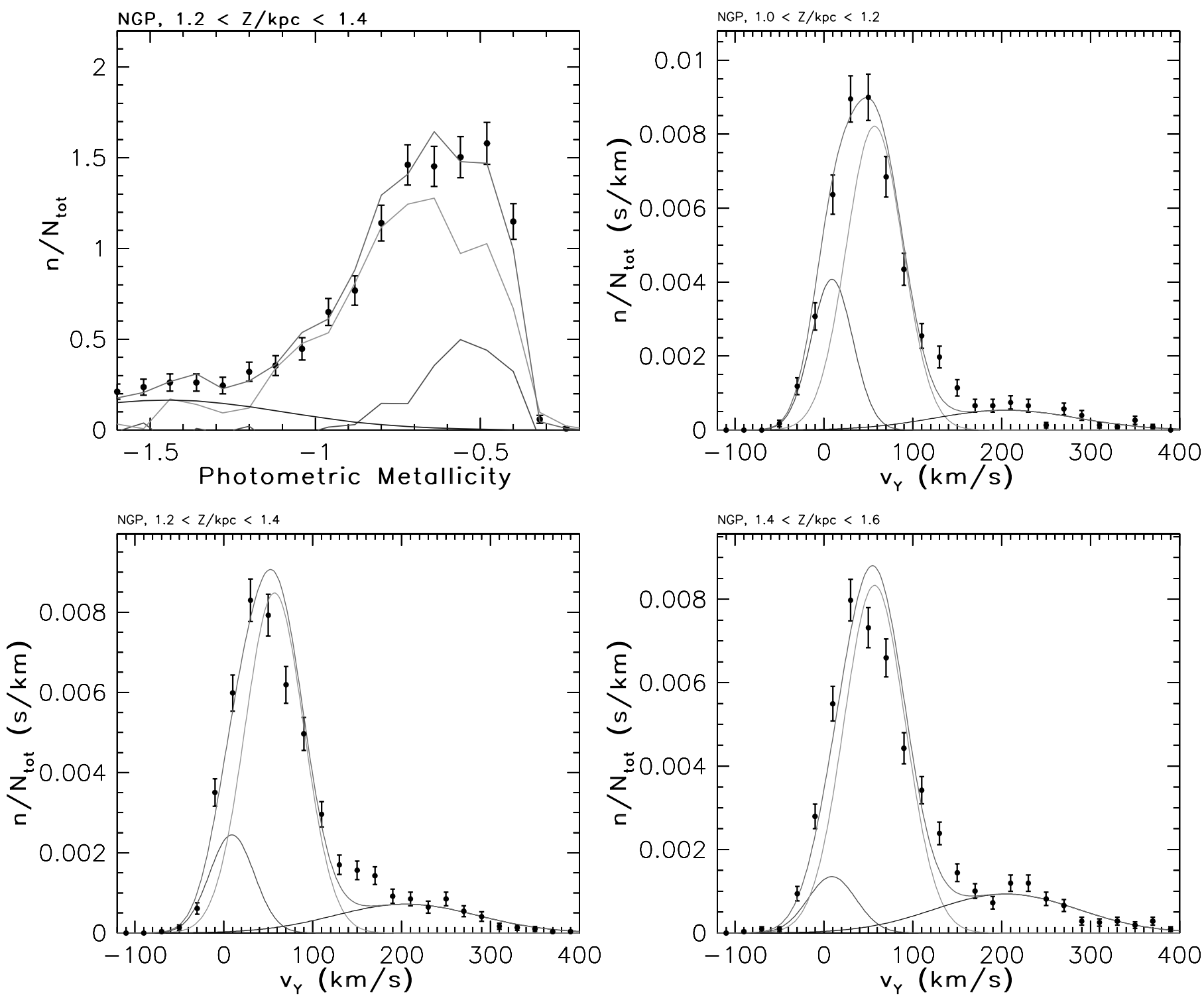

Fig. 15.- Top left panel: Test of the hypothesis that the observed variation of metallicity distribution with $|Z|$ is due to a varying mixing ratio of the independent thinand thick-disk metallicity distributions. The latter are determined using metallicity distributions in the $|Z|=1.0-1.2 \mathrm{kpc}$ and $|Z|=2.0-2.5 \mathrm{kpc}$ bins and are shown as lines (thin disk with a peak at roughly -0.5 and thick disk at roughly -0.7 ). They are scaled using the $4.6: 1$ thick-disk-to-thin-disk number ratio expected for the displayed $|Z|=1.2-1.4 \mathrm{kpc}$ bin from the counts profile. The data for this $|Z|$ bin are shown by symbols with error bars. The line rising toward the left shows halo contribution $(15 \%)$, and the top line is the sum of all three components. The remaining three panels show measured rotational velocity distributions in three $Z$ slices $($ symbols with error bars; $1.0-1.2 \mathrm{kpc}$ : top right; $1.2-1.4 \mathrm{kpc}$ : bottom left; $1.4-1.6 \mathrm{kpc}$ : bottom right). The lines with a peak at $\sim 220 \mathrm{~km} \mathrm{~s}^{-1}$ are Gaussians corresponding to halo stars, with the parameters listed in Table 5. The highest lines are the sums of halo contribution and the disk contribution. The latter is modeled as a sum of two Gaussians centered on $9 \mathrm{~km} \mathrm{~s}^{-1}$, and on $57 \mathrm{~km} \mathrm{~s}^{-1}$ (shown by thin lines), with widths listed in Table 5. The increase of their widths with $|Z|$ is consistent with estimated measurement errors; the implied intrinsic widths are $18 \mathrm{~km} \mathrm{~s}^{-1}$ for narrow Gaussians and $28 \mathrm{~km} \mathrm{~s}^{-1}$ for wide Gaussians. These lines are normalized according to thick-to-thin number ratio and halo contribution listed in Table 5. [See the electronic edition of the Journal for a color version of this figure.]

We seek metallicity and velocity distributions whose linear combination, with weights given by the observed spatial profiles, reproduces the variation of the observed distributions with $Z$. This can be done with only a minimal reliance on models because the data span a wide range of $Z$, and because there are clearly detected $Z$ gradients in both the metallicity and velocity distributions, which cannot be attributed to halo stars. The disk spatial profile (with the $\mathrm{J} 08$ best-fit parameters) implies that the $|Z|=$ $2.0-2.5 \mathrm{kpc}$ bin is dominated by thick-disk stars (only $2 \%$ of disk stars from this bin are expected to belong to the thin-disk component, with a $38 \%$ contribution from halo stars), while they contribute, for example, $\sim 67 \%$ of all disk stars in the $|Z|=0.8-$ $1.2 \mathrm{kpc}$ bin. Therefore, one can subtract the $\sim 38 \%$ halo contribution from the metallicity distribution in the $|Z|=2.0-2.5 \mathrm{kpc}$ bin, then renormalize the difference, and treat it as the thick-disk metallicity distribution. It can then be subtracted, after renormalization, from the disk-dominated metallicity distribution in the $|Z|=0.8-1.2 \mathrm{kpc}$ bin. The renormalized difference can be treated as a pure thin-disk distribution. The two distributions can then be linearly combined and compared to distributions observed in the intermediate bins.

The top left panel of Figure 15 shows the result of this comparison for the $|Z|=1.2-1.4 \mathrm{kpc}$ bin. The metallicity distributions for both components appear non-Gaussian, with a difference of their means of about 0.2 dex. Their expected linear combination reveals discrepancies with the data, but they are not overwhelming and could be due to uncertainties in the decomposition procedure itself. 
TABLE 5

Best-Fit Parameters for $p\left(v_{Y}\right)$, Shown in Figure 15

\begin{tabular}{|c|c|c|c|c|c|c|c|c|}
\hline $\begin{array}{l}|Z| \text { Range }^{\mathrm{a}} \\
\quad(\mathrm{kpc})\end{array}$ & $\begin{array}{l}\langle|Z|\rangle^{\mathrm{b}} \\
(\mathrm{kpc})\end{array}$ & $N^{\mathrm{c}}$ & $\langle g\rangle^{\mathrm{d}}$ & $\begin{array}{c}\sigma_{d 1}{ }^{\mathrm{e}} \\
\left(\mathrm{km} \mathrm{s}^{-1}\right)\end{array}$ & $\begin{array}{c}\sigma_{d 2}{ }^{\mathrm{f}} \\
\left(\mathrm{km} \mathrm{s}^{-1}\right)\end{array}$ & $\begin{array}{c}\sigma_{H}^{\mathrm{g}} \\
\left(\mathrm{km} \mathrm{s}^{-1}\right)\end{array}$ & $\begin{array}{l}f_{H}^{\mathrm{h}} \\
(\%)\end{array}$ & $r_{D D}^{\mathrm{i}}$ \\
\hline $1.0-1.2 \ldots \ldots \ldots \ldots \ldots \ldots$ & 1.11 & 1142 & 15.3 & 23 & 32 & 80 & 11 & 2.7 \\
\hline $1.2-1.4$ & 1.39 & 1480 & 15.6 & 25 & 33 & 81 & 15 & 4.6 \\
\hline $1.4-1.6 \ldots \ldots+\ldots \ldots \ldots-1$. & 1.50 & 1603 & 15.9 & 27 & 34 & 81 & 19 & 7.9 \\
\hline
\end{tabular}

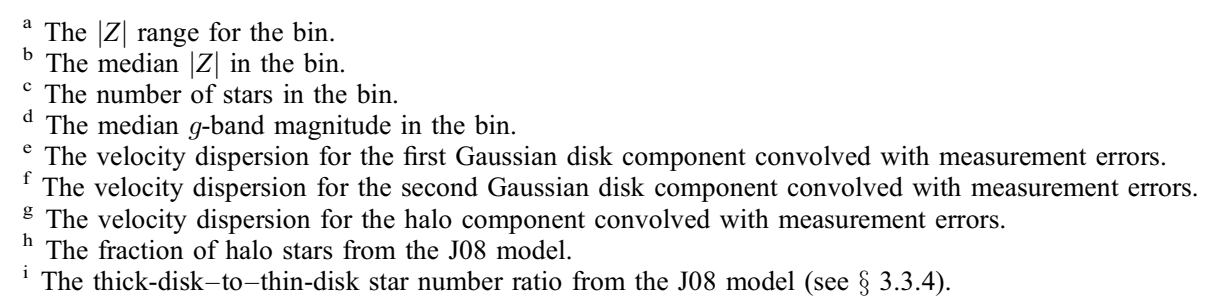

A similar linear decomposition method cannot be applied to the kinematic data analyzed here because the velocity measurement errors increase too much over the relevant range of $Z$. Instead, we choose to model the observed velocity distributions using two Gaussians, with fixed mean velocities and dispersions. The widths are convolved with known, $Z$-dependent, velocity measurement errors when fitting the four free parameters. We normalize the two Gaussians assuming their relative contributions (and halo normalization) predicted by the density (counts) profiles shown in Figure 6.

The best fits are shown in Figure 15 for three representative $Z$ bins, with the best-fit parameters listed in Table 5 . The presumed thick-disk Gaussian has a velocity lag of $48 \mathrm{~km} \mathrm{~s}^{-1}$, relative to the first Gaussian (centered at 57 and $9 \mathrm{~km} \mathrm{~s}^{-1}$, respectively). The implied intrinsic velocity dispersions are 18 and $28 \mathrm{~km} \mathrm{~s}^{-1}$. The fits are not as good as those shown in Figure 13, but the discrepancies are not too large. They are, however, formally highly statistically significant, due to the large number of stars, but there is always a possibility for hidden systematic errors. If the thickdisk Gaussian were replaced by a non-Gaussian velocity distribution, it is likely that the most egregious discrepancies around $v_{Y} \sim 150 \mathrm{~km} \mathrm{~s}^{-1}$ could be resolved.

Therefore, the marginal metallicity and velocity distributions do not strongly rule out the thin/thick-disk linear combination hypothesis, but only if both disks have non-Gaussian metallicity and velocity distributions. The choice for both metallicity and rotational velocity distributions is then between a linear combination of two fixed non-Gaussian distributions and a single nonGaussian distribution that slides with $Z$. A difficulty with the former hypothesis is that the implied thin-disk metallicity distribution has a mean of about -0.55 , which is too metal-poor by about 0.4 dex, compared to local measurements (Girardi \& Salaris 2001; Nordström et al. 2004). On the other hand, there is a worrisome possibility of a metallicity "compression" discussed in Appendix C, which could bias thin-disk measurements to lower values.

While fitting the marginal metallicity and kinematic distributions separately does not strongly discriminate among models, the thin/thick-disk linear combination hypothesis makes a very strong prediction about the joint two-dimensional distribution. Because the individual components are offset by $\sim 0.2$ dex in metallicity, and by $\sim 50 \mathrm{~km} \mathrm{~s}^{-1}$ in rotational velocity, this hypothesis predicts a correlation between metallicity and rotational velocity for samples selected from narrow $Z$ slices in the $Z$ range where neither component dominates. To compute the strength of this correlation, we generated a Monte Carlo sample for the
$Z=1.0-1.2 \mathrm{kpc}$ bin, where the correlation should be strong, with the same size as the observed sample (the expected thickdisk-to-thin-disk star number ratio is 2.7). The distributions of the data and model stars in the velocity versus metallicity plane are shown in Figure 16.

Even without any computation, it is evident from the bottom left panel of Figure 16 that the model distributions are strongly correlated: the $28 \%$ contribution of thin-disk stars, which have smaller mean velocity and higher metallicity than thickdisk stars, induces a net metallicity-velocity correlation (roughly $\left.-90 \mathrm{~km} \mathrm{~s}^{-1} \mathrm{dex}^{-1}\right)$. The value of Kendall's rank correlation coefficient (see, e.g., Lupton 1993) for the model distribution is $-0.30 \pm 0.04$, which is significantly inconsistent with the observed value of $0.017 \pm 0.018$ (we limit velocities to $120 \mathrm{~km} \mathrm{~s}^{-1}$ and metallicity to $[\mathrm{Fe} / \mathrm{H}]>-1$, to exclude halo stars).

In order to test whether this observed lack of correlation is localized to the north Galactic pole, we have also evaluated Kendall's rank correlation coefficient for stars with $Z=1.0-1.2 \mathrm{kpc}$ in three $10^{\circ}$ wide patches along the $l=180^{\circ}$ line and with $b=50^{\circ}, 30^{\circ}$, and $-50^{\circ}$. None of these patches exhibit a statistically significant correlation between velocity and metallicity, with the value of the correlation coefficient toward the north Galactic pole remaining the most significant. In order to test whether the measured correlation coefficient depends on the selection of the $Z$ bin, we evaluated it for seven $200 \mathrm{pc}$ thick bins in the range $Z=0.5-$ $2.1 \mathrm{kpc}$, and we found values statistically consistent with the value for the $Z=1.0-1.25 \mathrm{kpc}$ bin.

For another test, one that is less sensitive to errors in the adopted metallicity scale, we compared velocity histograms for stars with $-1<[\mathrm{Fe} / \mathrm{H}]<-0.8$ and $-0.6<[\mathrm{Fe} / \mathrm{H}]<-0.4$. The observed median rotation velocities differ by $1 \mathrm{~km} \mathrm{~s}^{-1}$, while the model values differ by $25 \mathrm{~km} \mathrm{~s}^{-1}$, or 8 times more than the expected statistical noise. To explain the observed flat median rotation velocity versus metallicity behavior as due to errors in adopted absolute magnitudes, adopted $M_{r}$ for stars with $-1<[\mathrm{Fe} / \mathrm{H}]<$ -0.8 would have to be too faint by $\sim 1 \mathrm{mag}$, and $\sim 1.5 \mathrm{mag}$ too bright for stars with $-0.6<[\mathrm{Fe} / \mathrm{H}]<-0.4$. Such large errors are not consistent with the constraints on the photometric parallax relation discussed in Appendix A, nor with plausible age effects.

These tests would be statistically even stronger if the samples extended to at least $Z=0.75 \mathrm{kpc}$, where the expected fractions of thin- and thick-disk stars are equal. This is not possible with the $b>80^{\circ}$ sample due to image saturation (at $r \sim 14$ ), while for stars observed at lower Galactic latitudes, the radial velocity measurement is required to compute the rotational velocity 

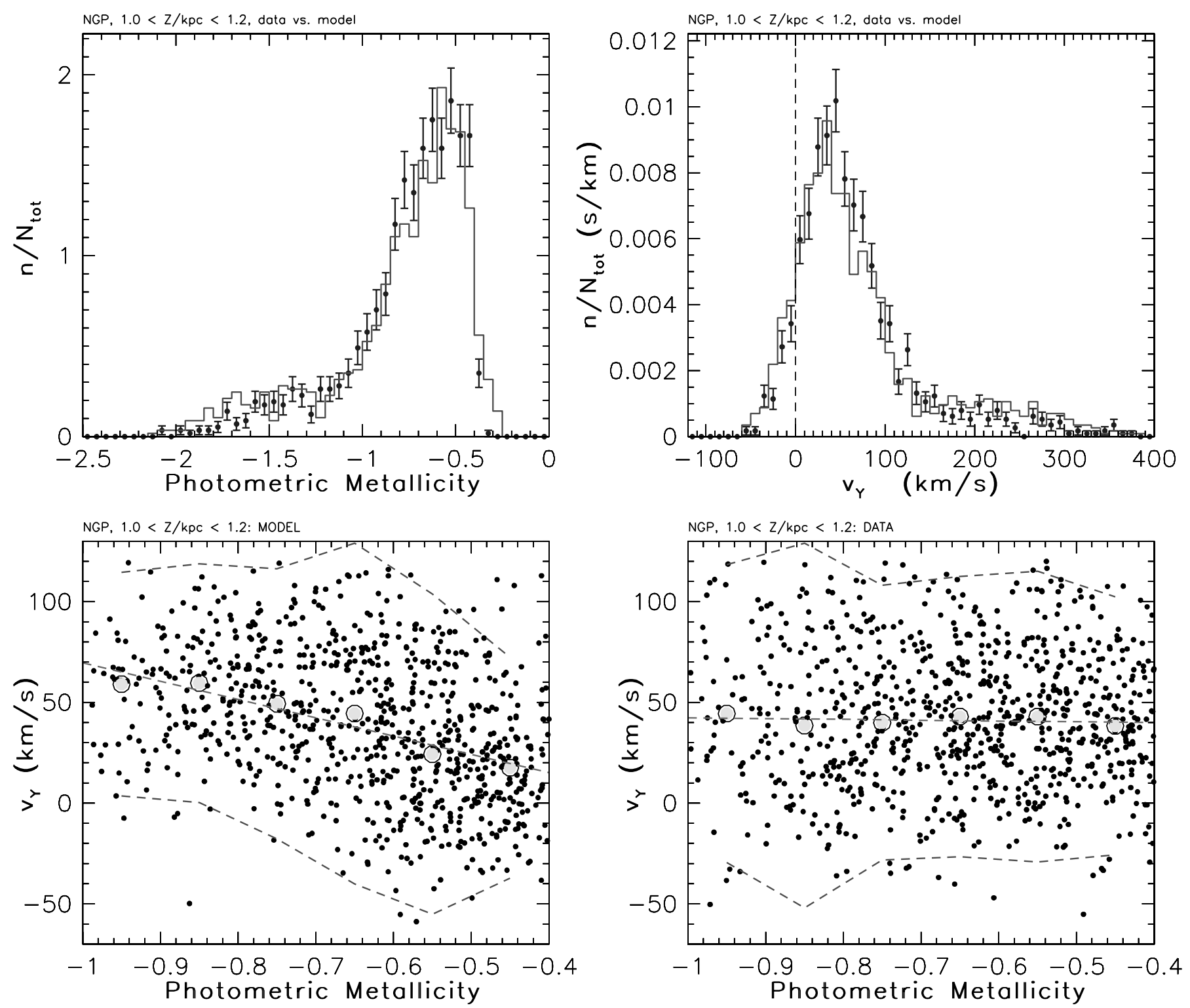

FIG. 16.-Comparison of data for 1142 stars with $b>80$ and $1.0<|Z| / \mathrm{kpc}<1.2 \mathrm{kpc}$, and a model based on a traditional disk decomposition into thin and thick components. The model assumes three Gaussians with contributions of thin-disk, thick-disk, and halo stars equal to $28 \%$, $52 \%$, and $20 \%$, respectively. The Gaussians

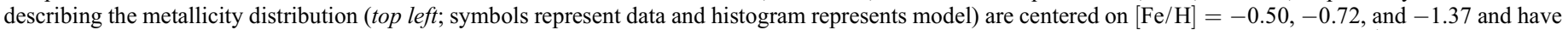

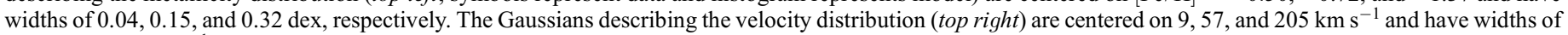

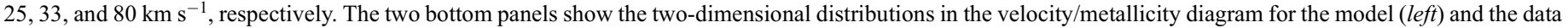

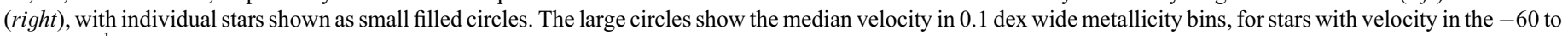

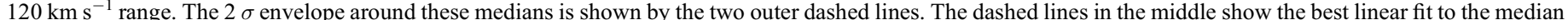
velocity, with slopes of $-91 \mathrm{~km} \mathrm{~s}^{-1} \mathrm{dex}^{-1}$ (model) and $-4.1 \mathrm{~km} \mathrm{~s}^{-1} \mathrm{dex}^{-1}$ (data). [See the electronic edition of the Journal for a color version of this figure.]

component. Hence, we use the SDSS spectroscopic survey to extend the sample to $Z<1 \mathrm{kpc}$. In addition, this sample also critically tests a possibility that these thin/thick-disk separation difficulties are caused by photometric metallicity problems.

Figure 17 shows the $v_{\Phi}$ versus $[\mathrm{Fe} / \mathrm{H}]$ velocity diagrams for two samples of stars with SDSS spectra that have $Z=600-800 \mathrm{pc}$ and $Z=800-1000 \mathrm{pc}$. Here $v_{\Phi}$ is the rotational velocity component corrected for the LSR and peculiar solar motion (toward the NGP, $v_{\Phi} \sim v_{Y}-225 \mathrm{~km} \mathrm{~s}^{-1}$ ). Although the metallicity distribution of the SDSS spectroscopic sample is highly biased (see the top right panel of Fig. 3), the dependence of the median rotational velocity for narrow metallicity bins, as a function of metallicity, is not strongly affected. These two subsamples are also inconsistent with the strong velocity-metallicity correlation expected from traditional thin-thick decomposition.
We conclude that the absence of a correlation between the observed velocity and metallicity distributions for disk stars represents a major problem for the interpretation of vertical velocity and metallicity gradients as being due to a varying linear combination of two fixed distributions.

Our conclusion does not contradict previous work on this subject because the older samples did not include simultaneous distance, velocity, and metallicity measurements for a sufficient number of stars with the appropriate distance distribution. A test based on a randomly selected 10 times smaller subsample, with photometric errors and proper-motion errors increased by a factor of 2, resulted in an inconclusive difference in Kendall's correlation coefficients between the "data" and a thin/thick-disk model.

It is noteworthy that Norris (1987) proposed a Galaxy model that does not assume that thin and thick disks are discrete components, 

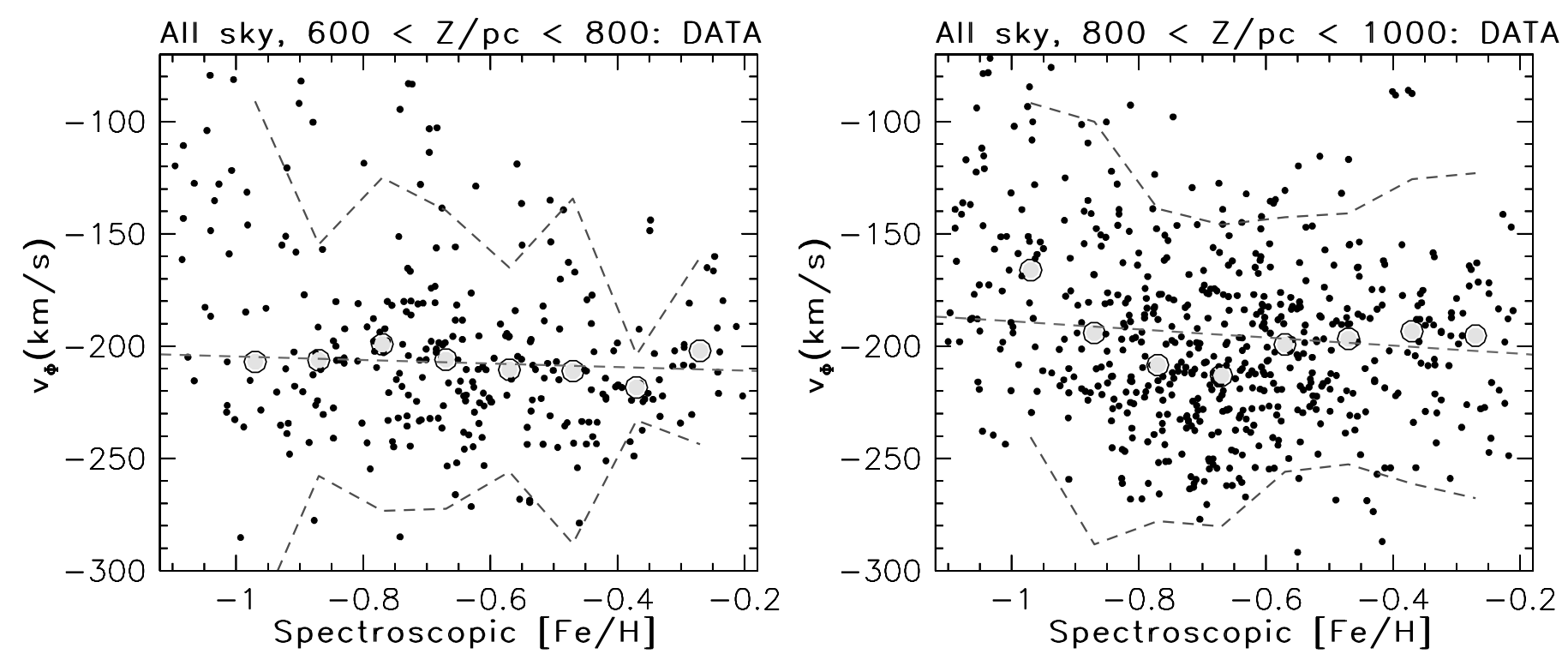

FIG. 17.- Analogous to the bottom right panel of Fig. 16, except that the SDSS spectroscopic sample of stars is used $\left(v_{Y}\right.$ from Fig. 16 corresponds to $\left.v_{\Phi}+225 \mathrm{~km} \mathrm{~s}^{-1}\right)$. Its all-sky distribution and radial velocity measurement enable the sampling of regions closer to the Galactic plane than with the $b>80^{\circ}$ sample $(l e f t: 285$ stars with $|Z|=$ $600-800 \mathrm{pc}$; right: 583 stars with $|Z|=800-1000 \mathrm{pc}$; both with $0.2<g-r<0.4)$. Although the metallicity distribution of the spectroscopic sample is highly biased (see the top right panel of Fig. 3), the dependence of the median rotational velocity for narrow metallicity bins, as a function of metallicity, is not strongly affected. Note the absence of a strong $v_{\Phi}$ vs. $|\mathrm{Fe} / \mathrm{H}|$ gradient $\left(\lesssim 20 \mathrm{~km} \mathrm{~s}^{-1} \mathrm{dex}^{-1}\right)$. [See the electronic edition of the Journal for a color version of this figure.]

but instead form a kinematical and chemical continuum. Stars traditionally associated with the thick disk belong to an "extended" disk (in terms of spatial distribution) in Norris's terminology and represent an extreme tail of metallicity and kinematic distributions. Our results appear roughly consistent with Norris's proposal and provide quantitative support for such a "continuous" view of the disk over the entire relevant range of distances from the Galactic plane.

\subsubsection{The "Metal-weak Thick Disk" Revisited}

In order to test whether the third Gaussian component discussed in $\S 3.3 .1$ has the same kinematics as the rest of disk stars, we compare the $v_{Y}$ histograms for $-1.1<[\mathrm{Fe} / \mathrm{H}]<-0.8$ and $-0.6<[\mathrm{Fe} / \mathrm{H}]<-0.5$ subsamples in two $Z$ bins: $0.5-1 \mathrm{kpc}$ and $1.5-2.0 \mathrm{kpc}$. We find no statistically significant differences, with an upper limit on the relative velocity offset of $\sim 15 \mathrm{~km} \mathrm{~s}^{-1}$. This kinematic similarity supports the view that stars with $-1.1<$ $[\mathrm{Fe} / \mathrm{H}]<-0.8$ reflect a non-Gaussian metallicity distribution of disk stars, rather than a separate entity (Beers et al. 2002). Further insight will be obtained from the C. Rockosi et al. (2008, in preparation) discussion of the properties of the metal-weak thick disk as revealed by the spectroscopic SEGUE sample.

\subsection{Spatially Localized Deviations from the Mean Metallicity Distribution}

The rich metallicity and kinematic data presented here enable more powerful searching for Milky Way substructure than possible using the stellar number density maps alone (see, e.g., J08). We utilize the large sky coverage of the DR6 catalog to quantify spatial deviations from the conditional metallicity distribution discussed in $\S \S 3.2$ and 3.3. We first constructed 51 maps, such as that shown in the top right panel of Figure 5, for regions defined by a $1 \mathrm{kpc}$ by $1 \mathrm{kpc}$ square footprint in the $X-Y$ plane. In each map, we compute the median metallicity in two $Z$ slices that are dominated by disk ( $1-2 \mathrm{kpc})$ and halo $(5-7 \mathrm{kpc})$ stars. The range of cylindrical radius, $R$, is $6-14 \mathrm{kpc}$ for the disk slice and 5$15 \mathrm{kpc}$ for the halo slice.
In the range $6<R / \mathrm{kpc}<10$, the median disk metallicity is $[\mathrm{Fe} / \mathrm{H}]=-0.72$, with an rms scatter of $0.05 \mathrm{dex}$ and a median distribution width of $0.27 \mathrm{dex}$. There is no discernible radial met-

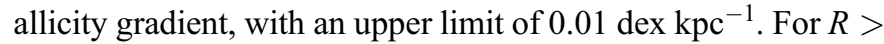
$10 \mathrm{kpc}$, the median metallicity is $[\mathrm{Fe} / \mathrm{H}]=-0.80$, with the shift of 0.08 dex probably due to stars from the Monoceros stream, as discussed below. The median halo metallicity is $[\mathrm{Fe} / \mathrm{H}]=$ -1.40 , with an rms scatter of 0.03 dex and the median distribution width of 0.41 dex. There is no discernible radial metallicity gradient, with an upper limit of $0.005 \mathrm{dex} \mathrm{kpc}^{-1}$.

The mean expected statistical scatter in the medians is $0.005 \mathrm{dex}$ for the disk and 0.011 dex for the halo (due to a wider distribution and fewer stars for the latter), suggesting that the variation of the median halo metallicity is probably insignificant, while the rms variation of the median disk metallicity of 0.05 dex appears real. The photometric calibration errors in individual SDSS runs, which could produce a metallicity scatter of a similar magnitude, are averaged out because many runs contribute to each map. Furthermore, such an instrumental effect is ruled out by the fact that the $u$-band calibration errors would have to have an rms of 0.02 mag to produce the disk median metallicity rms of $0.05 \mathrm{dex}$ and only $0.006 \mathrm{mag}$ to produce the halo rms of $0.03 \mathrm{dex}$. Hence, were the disk rms due to calibration errors, the halo rms would have been 0.1 dex and not 0.03 dex.

As an additional method to search for localized substructure, we subtracted a best-fit model from each map (such as those described in $\S 3.3$ ) and visually inspected residual maps. The only strong feature found in residual maps was localized at $Y \sim 0$, $Z \sim 3-4 \mathrm{kpc}$, and $R \sim 15 \mathrm{kpc}$ and represents an excess of $[\mathrm{Fe} / \mathrm{H}] \sim-1$ stars. It is clearly visible in the median metallicity $R Z$ map and, as an especially striking feature, in a conditional metallicity distribution map shown in Figure 18. Using its spatial position, we identify this feature as the Monoceros stream, ${ }^{39}$ discovered in SDSS data using stellar counts by Newberg et al. (2002).

\footnotetext{
39 Immediately following its discovery, it was not clear whether the Monoceros stellar overdensity was a ring, stream, or due to disk flaring. Subsequent work has demonstrated its streamlike profile; see, e.g., maps in J08.
} 

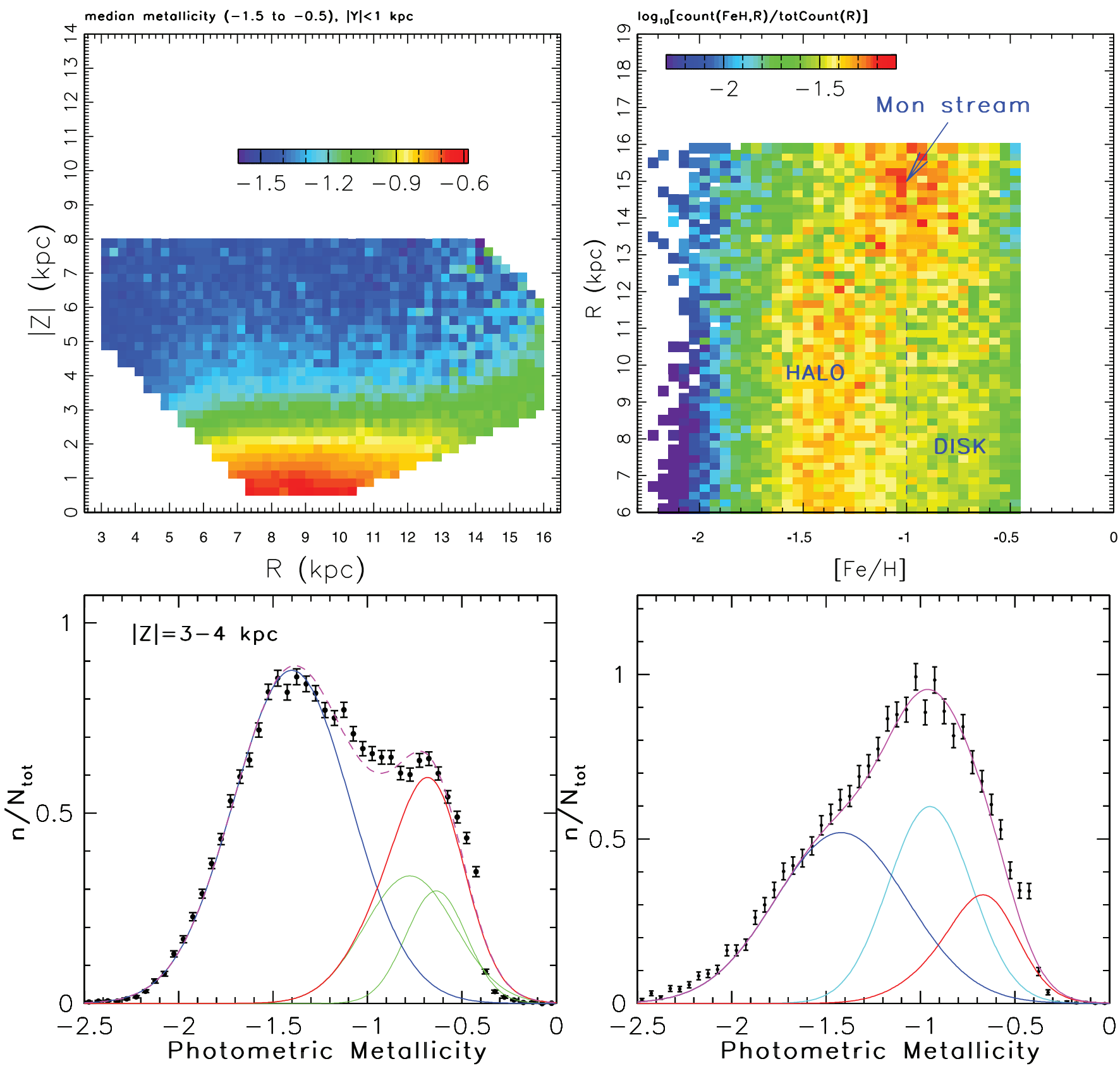

FIG. 18. - Top left panel: Dependence of the median photometric metallicity for $\sim 1.04$ million stars from SDSS DR6 with $14.5<r<20,0.2<g-r<0.4$, and $|Y|<1 \mathrm{kpc}$, in cylindrical Galactic coordinates $R$ and $|Z|$. This $Y$ range is selected to include the Monoceros stream, which represents an overdensity by a factor of $\sim 1.5-2$ in a region around $R \sim 15 \mathrm{kpc}$ and $|Z| \sim 3-4 \mathrm{kpc}$. As discernible from the map, this region has a larger median metallicity than expected for this $|Z|$ range from smaller $R$. Top right panel: Conditional metallicity probability distribution for a subsample of $\sim 111,000$ stars with $3<|Z| / \mathrm{kpc}<4$. The strong overdensity at $R>12 \mathrm{kpc}$ is the Monoceros stream. The bottom panels show the metallicity distribution (symbols with error bars) for a subsample of $\sim 40,000$ stars with $6<R / \mathrm{kpc}<9$ (left) and for $\sim 12,000$ stars with $13<R / \mathrm{kpc}<16$ (right). The lines have the same meaning as in Fig. 7, with the addition of the cyan line in the bottom right panel. This line is a 0.22 dex wide Gaussian centered on $[\mathrm{Fe} / \mathrm{H}]=-0.95$ and accounts for $33 \%$ of stars in the sample that presumably belong to the Monoceros stream (see $\S 3.5 .1$ for details).

\subsubsection{The Metallicity Distribution for the Monoceros Stream}

The conditional metallicity map from Figure 18 demonstrates that regions with $R<12$ are not strongly affected by the Monoceros stream. We compare the metallicity distributions for stars with $6<R / \mathrm{kpc}<9$ (control sample) and for stars with $13<R / \mathrm{kpc}<16$ in the bottom panels of Figure 18 . The metallicity distribution of the control sample is consistent with the halo and disk metallicity distributions described in $\S 3.3 .1$, with a few minor adjustments: the disk distribution is shifted by $0.07 \mathrm{dex}$ and the halo distribution by 0.02 dex, toward higher metallicity; the fraction of halo stars is changed from $61 \%$ to $55 \%$; and 0.16 dex is added in quadrature to the widths of the three Gaussians to account for the increased metallicity errors in single-epoch DR6 data.

The subsample containing the Monoceros stream can be described using the same function as for the control sample and an additional 0.22 dex wide Gaussian component centered on $[\mathrm{Fe} / \mathrm{H}]=-0.95$, with a relative normalization of $33 \%$. When corrected for measurement errors, the implied width of the metallicity 
distribution for the Monoceros stream is $0.15 \mathrm{dex}$. The best-fit normalization is in good agreement with spatial profiles from J08, which suggests that the Monoceros stream is about a factor of 2 overdensity over the local background counts (i.e., a relative normalization of $50 \%$ ).

\subsubsection{The Kinematics of the Monoceros Stream}

We select a subsample of $\sim 11,000$ stars that maximizes the fraction of stars from the Monoceros stream and allows an estimate of rotational velocity using only longitudinal proper motion, by requiring $|Y|<1 \mathrm{kpc}, 3<Z / \mathrm{kpc}<4,13<R / \mathrm{kpc}<16$, and $170^{\circ}<l<190^{\circ}$. The distribution of these stars in the rotational velocity versus metallicity diagram is shown in the top right panel of Figure 19. It is discernible that the Monoceros stream has kinematics more similar to disk stars than to halo stars. We obtain a more accurate assessment with the aid of an analogous map for a control sample selected using similar criteria, except that $9<R / \mathrm{kpc}<12$ (top left panel). The difference of these two maps is shown in the bottom left panel.

The excess stars are centered on $[\mathrm{Fe} / \mathrm{H}] \sim-1$, as expected from the best fit described above. Their velocity distribution shows a strong peak at $v_{l} \sim v_{Y} \sim-50 \mathrm{~km} \mathrm{~s}^{-1}$, with a long tail toward more positive velocities. This residual map indicates that most of the Monoceros stream stars move in the direction of LSR rotation with velocities of up to $\sim 270 \mathrm{~km} \mathrm{~s}^{-1}$. This result is qualitatively in agreement with Penarrubia et al. (2005), who ruled out retrograde motions using models and proper-motion measurements.

The latitudinal velocity, $v_{b}$, based on the latitudinal propermotion component, is a linear combination of the radial and vertical velocity components. The median latitudinal velocity of stars from the Monoceros stream region in the rotational velocity versus metallicity plane is shown in the bottom right panel of Figure 19. There is no significant offset from $0\left(<20 \mathrm{~km} \mathrm{~s}^{-1}\right)$ in parts of the diagram where the excess of the Monoceros stream stars is the highest. This presumably indicates that contributions from the radial and vertical motion for the Monoceros stream stars cancel (for disk stars, the medians for both components should be 0 ). Together with the radial velocity measurements obtained by Conn et al. (2005) and Martin et al. (2006), this information can be used to further refine models, such as those described by Penarrubia et al. (2005).

\section{DISCUSSION AND CONCLUSIONS}

The spectroscopic stellar parameters for over $60,000 \mathrm{~F}$ and G dwarfs, computed by the SEGUE Stellar Parameter Pipeline (Beers et al. 2006; Allende Prieto et al. 2006, 2007; Lee et al. 2007a, 2007b) using the SDSS spectroscopic database, allowed us to derive photometric estimators for effective temperature and metallicity in the SDSS photometric system. The availability of the SDSS imaging survey, with its accurate ugr photometry, then enabled an unbiased volume-limited study of the stellar metallicity distribution to a distance limit of $\sim 10 \mathrm{kpc}$.

\subsection{Photometric Estimates for Effective Temperature and Metallicity}

The photometric metallicity estimator based on the SDSS $u-g$ and $g-r$ colors is reminiscent of the traditional $\delta(U-B)_{0.6}$ method based on the $U B V$ photometry. It reflects the same physics and can be applied to F- and G-type main-sequence stars $(0.2<g-r<0.6)$. For the SDSS single-epoch main survey data, it provides metallicity accurate to 0.2 dex or better for stars brighter than about $g=19.5$. In this magnitude and color range, the photometric effective temperature estimator reproduces spectroscopic temperature with an rms scatter of only $\sim 100 \mathrm{~K}$. The accuracy ${ }^{40}$ of $100 \mathrm{~K}$ for effective temperature, and $0.2 \mathrm{dex}$ or better for metallicity, is comparable to parameter accuracy achieved using artificial neural networks with spectroscopic observations (Snider et al. 2001) and the estimated accuracy of parameters determined from SDSS spectra (Beers et al. 2006). It is plausible that increased photometric accuracy would further improve these values. For example, photometry accurate to $1 \%$ may enable MK spectral type determination with errors smaller than one subtype. Derived mapping from color space to metallicity implies that, at least formally, an error in the $u-g$ color of $0.01 \mathrm{mag}$ induces a metallicity error that varies from $0.01 \mathrm{dex}$ at $[\mathrm{Fe} / \mathrm{H}]=-0.5$ to $0.06 \mathrm{dex}$ at $[\mathrm{Fe} / \mathrm{H}]=-1.5$. This is as good a performance as obtained by the Strömgren $u v b y \beta$ narrowband photometric system that was optimized for this purpose (e.g., Strömgren 1966; Nordström et al. 2004). In other words, the increase of metallicity errors due to increased bandpass width can be compensated for by an improved photometric accuracy.

We apply these methods to a photometric catalog of co-added SDSS observations from the so-called Stripe 82 (Ivezić et al. 2007a). These deeper and photometrically exceedingly precise co-added data allowed us to measure an unbiased metallicity distribution for a volume-limited sample of $\sim 200,000 \mathrm{~F}$ - and G-type stars in the $0.5-8 \mathrm{kpc}$ distance range. We also study the metallicity distribution using a shallower, but much larger, sample of several million stars in $8500 \mathrm{deg}^{2}$ of sky provided by SDSS DR6. The large sky coverage tests the conclusions derived using the relatively small Stripe 82 sample and enables detection of coherent substructures in the kinematics-metallicity space, such as the Monoceros stream.

\subsection{The Milky Way Structure and Multidimensional Stellar Counts}

From an observer's point of view, the ultimate goal of Milky Way studies is to measure and describe the distribution (counts) of stars in the space spanned by apparent brightness, colors in multiple bandpasses, ${ }^{41}$ two proper-motion components $\left(\mu_{l}, \mu_{b}\right)$, radial velocity $\left(v_{\text {rad }}\right)$, and position on the sky. Specialized to SDSS data, we seek to understand the eight-dimensional probability density function, $p\left(g, u-g, g-r, \mu_{l}, \mu_{b}, v_{\text {rad }}, l, b\right)$. This function could be described empirically, without any reference to stellar and Galactic structure, but in practice measurements are used to place constraints on the latter. From a theorist's point of view, the problem of interpreting data can be rephrased as follows: given a small control volume centered on $(R, Z, \phi),(1)$ what is the distribution of stars as a function of luminosity ${ }^{42} L,(2)$ what is the metallicity distribution for a given $L$, and (3) what are the distributions of three velocity components, $v_{\phi}, v_{R}$, and $v_{Z}$,

\footnotetext{
${ }^{40}$ Here precision may be a more appropriate terminology than accuracy because the photometric estimates are tied to SDSS spectroscopic values rather than to an absolute metallicity scale. That is, all systematic errors in SDSS spectroscopic parameters are inherited by photometric estimators.

${ }^{41}$ We limit this analysis to two colors, $u-g$ and $g-r$, which provide good estimates of effective temperature and metallicity for $\mathrm{F}$ and $\mathrm{G}$ main-sequence stars. However, an alternative is to consider a full spectral shape, instead of colors, which carries all the relevant information about the three main stellar parameters $\left(T_{\text {eff }}\right.$ $\log g,[\mathrm{Fe} / \mathrm{H}])$.

42 At least in principle, it would be desirable to measure the stellar luminosity function, and other quantities, as a function of stellar age, but estimating age for individual stars is very difficult (e.g., Nordström et al. 2004; Jorgensen \& Lindegren 2005) and beyond the scope of this paper.
} 

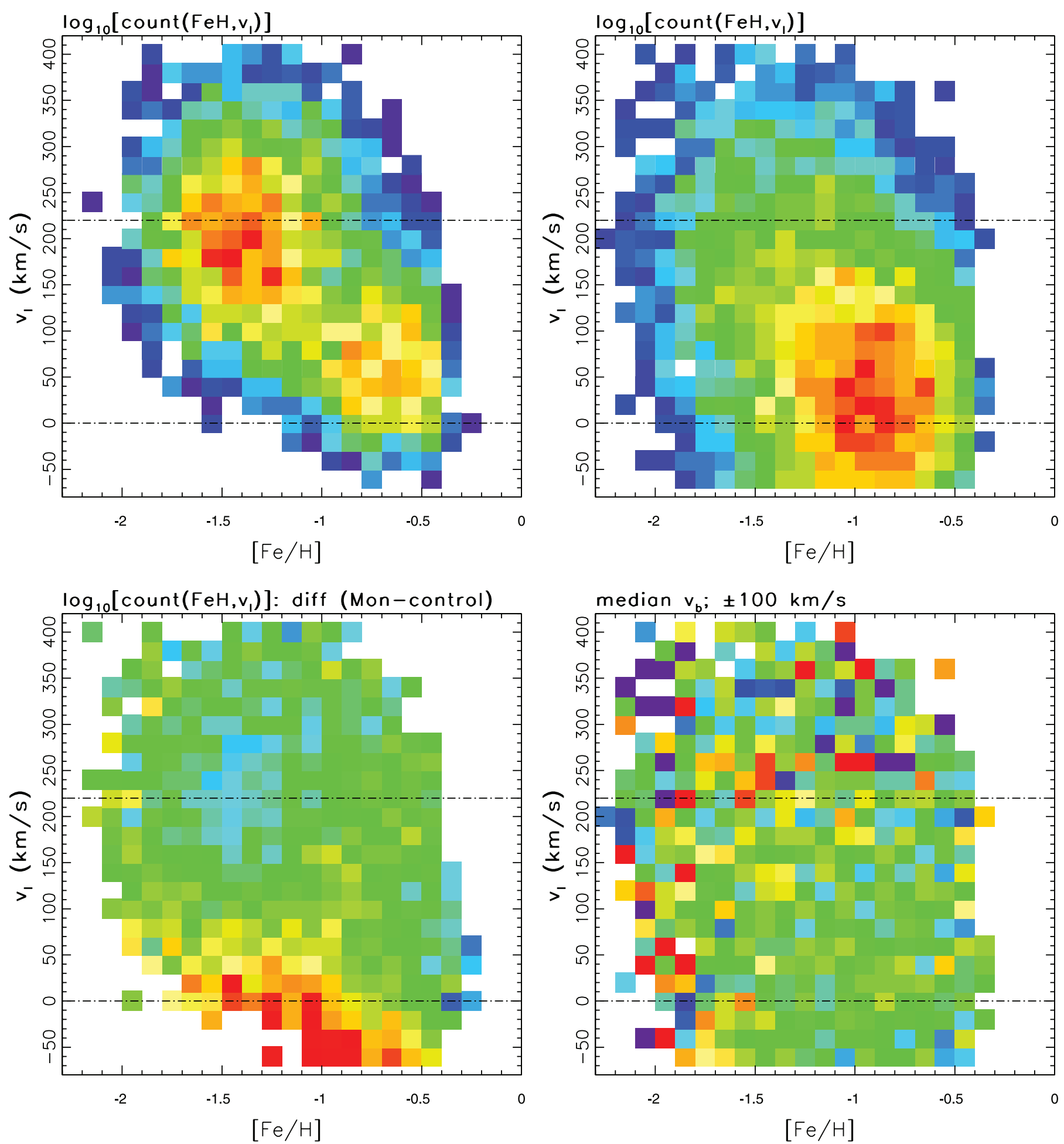

Fig. 19.-Top left panel: Distribution of $\sim 7200$ stars (logarithm of counts in each bin) with $|Y|<1 \mathrm{kpc}, 3<|Z| / \mathrm{kpc}<4,9<R / \mathrm{kpc}<12$, and $170^{\circ}<l<190^{\circ}$, in the longitudinal velocity vs. photometric metallicity plane (a slice through the map shown in the top right panel of Fig. 18). The longitudinal velocity, $v_{l}$, is based on propermotion measurements and at selected $l \sim 180^{\circ}$ corresponds to the rotational component (heliocentric). The top right panel is analogous, except that $\sim 12,000$ stars with $170^{\circ}<l<190^{\circ}$ are selected from the $13<R / \mathrm{kpc}<16$ radial range, which maximizes the fraction of stars from the Monoceros stream (clustered around $[\mathrm{Fe} / \mathrm{H}] \sim-1$ and $\left.v_{l} \sim 25 \mathrm{~km} \mathrm{~s}^{-1}\right)$. The difference of these two maps is shown in the bottom left panel. The bottom right panel shows the median latitudinal velocity $\left(v_{b}\right)$ for stars in the latter (Monoceros) subsample ( $\pm 100 \mathrm{~km} \mathrm{~s}^{-1}$ stretch; green corresponds to $0 \mathrm{~km} \mathrm{~s}^{-1}$ ). The analogous map for the $9<R /$ kpc $<12$ subsample appears similar. 
for a given $L$ and metallicity? Guided by these questions, we can write

$$
\begin{aligned}
p(g, u-g, g- & \left.r, \mu_{l}, \mu_{b}, v_{\mathrm{rad}}, l, b\right) \\
= & p_{1}(g, g-r \mid l, b) p_{2}(u-g \mid g, g-r, l, b) \\
& \times p_{3}\left(\mu_{l}, \mu_{b}, v_{\mathrm{rad}} \mid u-g, g, g-r, l, b\right),
\end{aligned}
$$

where the three functions on the right-hand side of equation (17) are discussed in this and two companion papers:

1. The function $p_{1}(g, g-r \mid l, b)$ describes the behavior of the $g$ versus $g-r$ color-magnitude (Hess) diagram as a function of position on the sky. This behavior is a reflection of stellar luminosity function, $\Phi(L)$, and the dependence of stellar number density on spatial coordinates, $\rho(R, Z, \phi)$. A best-fit model for $\rho(R, Z, \phi)$ developed by $\mathrm{J} 08$ is discussed in $\S$ 3.3.4.

2. The function $p_{2}(u-g \mid g, g-r, l, b)$ describes the $u-g$ color distribution for a given bin in the $g$ versus $g-r$ colormagnitude diagram, and as a function of position on the sky. The $u-g$ color distribution reflects the metallicity distribution, $p([\mathrm{Fe} / \mathrm{H}] \mid R, Z, \phi)$, and $\rho(R, Z, \phi)$. In this contribution, we show that, similarly to $\rho(R, Z, \phi), p([\mathrm{Fe} / \mathrm{H}] \mid R, Z, \phi)$ can be well described (apart from local overdensities) as a sum of two components

$$
\begin{aligned}
p(x=[\mathrm{Fe} / \mathrm{H}] \mid R, Z, \phi)= & {\left[1-f_{H}(R, Z)\right] p_{D}(x \mid Z) } \\
& +f_{H}(R, Z) p_{H}(x),
\end{aligned}
$$

where the halo-to-disk counts ratio is simply $f_{H}(R, Z)=\rho_{H}(R, Z) /$ $\left[\rho_{D}(R, Z)+\rho_{H}(R, Z)\right]$.

The halo metallicity distribution, $p_{H}([\mathrm{Fe} / \mathrm{H}])$, can be modeled as a spatially invariant Gaussian centered on $[\mathrm{Fe} / \mathrm{H}]=-1.46$ (for the Stripe 82 catalog; for the full DR6 sample, the median $[\mathrm{Fe} / \mathrm{H}]=-1.40$ ) and with the intrinsic (not including measurement errors) width $\sigma_{H}=0.30 \mathrm{dex}$. For $|Z| \lesssim 10 \mathrm{kpc}$, an upper limit on the halo radial metallicity gradient is $0.005 \mathrm{dex} \mathrm{kpc} \mathrm{kp}^{-1}$.

The metallicity distribution of the disk component has an rms scatter of 0.16 dex, with the median varying as

$$
\mu_{D}(Z)=-0.78+0.35 \exp (-|Z| / 1.0 \mathrm{kpc}) \mathrm{dex},
$$

at $|Z|>0.5 \mathrm{kpc}$. In the $|Z|=1.0-1.5 \mathrm{kpc}$ range, the median metallicity is consistent with the measurements by Gilmore \& Wyse (1985). For $|Z| \lesssim 5 \mathrm{kpc}$, an upper limit on the disk radial metallicity gradient is $0.010 \mathrm{dex} \mathrm{kpc}{ }^{-1}$. The shape of the metallicity distribution of the disk component is non-Gaussian and can be modeled as

$$
\begin{aligned}
p_{D}(x=[\mathrm{Fe} / \mathrm{H}] \mid Z)= & 0.37 G[x \mid \mu=a(Z)+0.14, \sigma=0.11] \\
& +0.63 G[x \mid \mu=a(Z), \sigma=0.21],
\end{aligned}
$$

where the position $a(Z)$ and the median $\mu_{D}(Z)$ are related via $a(Z)=\mu_{D}(Z)-0.067$ (unless measurement errors are very large). These results represent powerful new constraints for the Galaxy formation and chemical evolution models (e.g., Tinsley 1975; Pagel \& Patchett 1975; Wyse \& Gilmore 1995 and references therein).

3. The function $p_{3}\left(\mu_{l}, \mu_{b}, v_{\text {rad }} \mid u-g, g, g-r, l, b\right)$ describes proper-motion and radial velocity measurements for a given bin in the $g$ versus $g-r$ color-magnitude diagram, as a function of position on the sky, and as a function of the $u-g$ color. This function locally reflects the behavior of the velocity ellipsoid, but
SDSS data probe sufficiently large distances to detect its spatial variation, as discussed in detail by B08. They find that the detailed behavior of kinematics can also be well described (apart from local overdensities) as a sum of two components, disk and halo, that map well to components detected in spatial profiles and metallicity distribution. The nonrotating halo component has by and large spatially uniform kinematics (in an overall sense, e.g., B08 discuss several kinematically coherent structures), while the disk kinematics is dominated by a vertical $(Z)$ gradient. The mean rotational velocity and the three velocity dispersions for disk stars can be modeled as relatively simple functions of the form $a+$ $b|Z|^{c}$, as discussed in detail by B08 (see also Girard et al. 2006). The shape of the rotational velocity distribution for the disk component is non-Gaussian and can be modeled, in the $|Z|=0.8-$ $5.0 \mathrm{kpc}$ range (and $R \sim 8 \mathrm{kpc}$ ), as

$$
\begin{aligned}
p_{D}\left(x=v_{\Phi} \mid Z\right)= & 0.25 G\left[x \mid \mu=v_{n}(Z), \sigma=12\right] \\
& +0.75 G\left[x \mid \mu=v_{n}(Z)+34, \sigma=34\right],
\end{aligned}
$$

where

$$
v_{n}(Z)=-3+19.2|Z / \mathrm{kpc}|^{1.25} \mathrm{~km} \mathrm{~s}^{-1} .
$$

We reiterate that the widths listed for $p_{D}([\mathrm{Fe} / \mathrm{H}] \mid Z)$ and $p_{D}\left(v_{\Phi} \mid Z\right)$ are intrinsic widths and the listed widths are measured with a relative accuracy of $\sim 10 \%$.

These functions provide a good description of the overall features in the distribution of stars in the spatial-kinematicmetallicity space, as observed by SDSS. Qualitatively, these results are in fair agreement with previous work (e.g., Gilmore \& Wyse 1985; Gilmore et al. 1989; Majewski 1993; Nordström et al. 2004; Girard et al. 2006). Quantitatively, the availability of SDSS data is enabling unprecedentedly powerful and robust studies, not only due to its large volume, but also thanks to its accurate and diverse measurements. In particular, with the SDSS data, the reach of massive statistical studies can now be extended from $<100$ pc (the Hipparcos distance limit; e.g., Dehnen \& Binney 1998; Nordström et al. 2004) to $\sim 10 \mathrm{kpc}$.

The results presented here are only a brief illustration of the great scientific potential of the SDSS stellar spectroscopic database. This data set will remain a cutting edge resource for a long time because other major ongoing and upcoming stellar spectroscopic surveys either are shallower (e.g., RAVE, $9<I<12$ ) or have a significantly narrower wavelength coverage and depth (Gaia, $r \lesssim 17$ ).

\subsubsection{Is There a Thick Disk?}

Perhaps the most significant result of our study, in addition to detection of the abundant substructure in metallicity space, is that the transition from the thin to the thick disk, seen (and originally defined by Gilmore \& Reid 1983) as an abrupt change of slope in the $\log$ (counts) versus $Z$ plot around $|Z| \sim 1 \mathrm{kpc}$, can be modeled as smooth shifts of metallicity and velocity distributions that do not change their shape. More quantitatively, using the above notation, the disk metallicity and velocity distribution can be described as

$$
\begin{aligned}
p_{D}\left(x=v_{\Phi} \text { or }[\mathrm{Fe} / \mathrm{H}] \mid Z\right)= & n_{1}(Z) G\left[x \mid \mu_{1}(Z), \sigma_{1}\right] \\
& +n_{2}(Z) G\left[x \mid \mu_{2}(Z), \sigma_{2}\right] .
\end{aligned}
$$

Traditionally, the two components are interpreted as thin and thick disks, and $n_{1}(Z)$ and $n_{2}(Z)$ are constrained by stellar number 
counts. They are modeled as exponential functions with scale heights of $\sim 300$ and $\sim 1000 \mathrm{pc}$, with $\mu_{1}(Z)$ and $\mu_{2}(Z)$, typically assumed independent of $Z$. This description is only mildly inconsistent with the observed marginal metallicity and velocity distributions. However, when the two distributions are analyzed simultaneously, this decomposition faces a serious difficulty. Because it combines different metallicity and velocity distributions for thin- and thick-disk components (the data require offsets of 0.2 dex and $48 \mathrm{~km} \mathrm{~s}^{-1}$ ), it predicts a strong and detectable correlation between them. The data presented here do not display any significant correlation and rule out this prediction at a highly significant level $(\sim 8 \sigma)$.

We find an alternative interpretation that does not imply a strong correlation between metallicity and velocity distributions. Formally, we find that the data can be fitted with $n_{1}$ and $n_{2}$ that do not vary with $Z$ (eqs. [20] and [21]), while $\mu_{1}$ and $\mu_{2}$ are coupled and vary with $Z$ according to equations (19) and (22). This ability to describe the disk metallicity and velocity distributions using functions with universal $Z$-independent shapes has fundamental implications for its origin: instead of two distinct components, our data can be interpreted with a single disk, albeit with metallicity and velocity distributions more complex than a Gaussian (note that the data require non-Gaussian distributions even in the traditional interpretation). While the disk separation into thin and thick components may still be a useful concept to describe the fairly abrupt change of number density around $|Z| \sim 1 \mathrm{kpc}$ (which is detected beyond doubt; see J08 for SDSS results), the disk spatial profile may simply indicate a complex structure (i.e., not a single exponential function), rather than two distinct entities with different formation and evolution history. If this is correct, then our results imply that different processes led to the observed metallicity and velocity distributions of disk stars, rather than a single process, such as mergers or an increase of velocity dispersion due to scattering, that simultaneously shaped both distributions.

On the other hand, it appears that stars from the solar neighborhood, believed to be thick-disk stars because of their kinematic behavior, have larger $\alpha$-element abundances, at the same $[\mathrm{Fe} / \mathrm{H}]$, than do thin-disk stars (e.g., Fuhrmann 2004; Bensby et al. 2003; Feltzing 2006; Reddy et al. 2006; Ramírez et al. 2007). The thick-disk stars, again selected kinematically, appear older than the thin-disk stars (e.g., Fuhrmann 2004; Bensby et al. 2003). Thus, it is possible that the data presented here are insufficient to distinguish detailed elemental and age differences and that highresolution spectroscopy is required to do so. If such supplemental data were available, for example, for the $\sim 20,000$ stars analyzed in Figures 7 and 13, one could determine whether the distributions of individual $\alpha$-elements admit a universal shape, and whether they are correlated with kinematics. These stars are confined to several hundred square degrees of sky, with a sky density of $\sim 100 \mathrm{deg}^{-2}$, and those at $|Z|<4 \mathrm{kpc}$ have $g \lesssim 18$. Such an undertaking is within the easy reach of modern spectrographs installed on $10 \mathrm{~m}$ class telescopes. High-resolution studies of slightly brighter subsets of stars are planned to be undertaken with the APOGEE subsurvey, part of the proposed next extension of the SDSS, SDSS-III.

\subsubsection{Multidimensional Substructure}

The samples discussed here are sufficiently large to constrain the global behavior of the metallicity distribution and to search for anomalies. The halo metallicity distribution is remarkably uniform. The rms scatter in the median value for $2 \mathrm{kpc}^{3}$ large bins of only 0.03 dex is consistent with expected statistical noise. The median disk metallicity in $1 \mathrm{kpc}^{3}$ bins in the $Z=1-2 \mathrm{kpc}$ range exhibits a statistically significant, but still fairly small, rms scatter of $0.05 \mathrm{dex}$. We detect a vertical metallicity gradient for disk stars $\left(0.1-0.2 \mathrm{dex} \mathrm{kpc}^{-1}\right)$, but radial gradients are limited to $\lesssim 0.01 \mathrm{dex} \mathrm{kpc}^{-1}$ for both disk and halo components, outside of regions with strong substructure.

The strongest overdensity identified in the metallicity space is the Monoceros stream. Its metallicity distribution is distinct from those for both halo and disk and has a similar width as the metallicity distribution of disk stars $(\sim 0.15 \mathrm{dex})$. Hence, recent discoveries of abundant substructure in stellar spatial distribution and kinematics are now extended to metallicity space. We concur with Nordström et al. (2004) that "the Galaxy is a far more complicated and interesting subject than ever before."

\subsubsection{Implications for Future Imaging Surveys}

The analysis and conclusions presented here are relevant for upcoming large-scale deep optical surveys such as the Dark Energy Survey (Flaugher et al. 2007), Pan-STARRS (Kaiser et al. 2002), and the Large Synoptic Survey Telescope (LSST; Tyson 2002). Of these, only LSST plans to obtain data in the $u$ band. $^{43}$ Over its $10 \mathrm{yr}$ long lifetime, the LSST survey will obtain about 70 observations in the $u$ band of a 20,000 deg 2 area (see Table 1 in Ivezić et al. 2008). Thanks to its large aperture, the median $5 \sigma$ depth of $\sim 24$ (for point sources) will be significantly fainter than for SDSS data (22.5), and the co-added data will reach a $5 \sigma$ depth of $u=26$. The potential of the photometric metallicity estimator for studying the evolution and structure of the Milky Way demonstrated here bodes well for the LSST science mission.

Using SDSS data, we estimate the number of stars for which LSST will provide metallicity measurements. Based on the discussion presented in $\S 2$, we adopt an error in the $u-g$ color of $0.05 \mathrm{mag}$ as a practical limit for robust metallicity studies. This color error corresponds to a metallicity error of 0.1 dex for metalrich stars and 0.2 dex for metal-poor stars. The LSST data will achieve this color accuracy for stars with $0.2<g-r<0.6$ if $g<23.5$. This is about 4 mag deeper than the analogous limit for the SDSS survey. Based on the counts of SDSS stars, we estimate that LSST will measure metallicity accurate to 0.2 dex or better ${ }^{44}$ for at least 200 million $\mathrm{F} / \mathrm{G}$ main-sequence stars brighter than $g=23.5$ (without accounting for the fact that stellar counts greatly increase close to the Galactic plane). For these stars, ${ }^{45}$ LSST will also provide proper-motion measurements accurate to about 0.2 mas yr $^{-1}$ at $g=21$ and 0.5 mas yr $^{-1}$ at $g=23$ (about 10 times more accurate and $\sim 3$ mag deeper than the SDSS-POSS catalog by Munn et al. [2004] used in this work). This data set will represent a deep complement to the Gaia mission $(g \lesssim 20$; Perryman et al. 2001; Wilkinson et al. 2005) and will enable detailed exploration of the Milky Way halo in a six-dimensional space spanned by three spatial coordinates, two velocity components, and metallicity, within a distance limit of $\sim 100 \mathrm{kpc}$. This study can be regarded as one of the first steps in this mapping endeavor, which is bound to provide unprecedented clues about the formation and

\footnotetext{
43 The LSST science requirements document is available from http://www .1sst.org/Science/lsst_baseline.shtml.

44 At the bright end, LSST color errors will be $<0.01 \mathrm{mag}$. An error of $0.01 \mathrm{mag}$ in the $g-r$ color corresponds to a $50 \mathrm{~K}$ random error in effective temperature, and an error of $0.01 \mathrm{mag}$ in the $u-g$ color corresponds to a random metallicity error of 0.01 dex at $[\mathrm{Fe} / \mathrm{H}]=-0.5$ and 0.05 dex at $[\mathrm{Fe} / \mathrm{H}]=-1.5$.

45 The 200 million stars from the "metallicity" sample will be observed over 250 times in the $g$ and $r$ bands with signal-to-noise ratios of about 20 or larger per observation even at the faint end (and the final error in the $g-r$ color below $1 \%$ ). The total number of stars that will be detected by LSST is of the order of 10 billion.
} 
evolution of our Galaxy. Indeed, "these are exciting times to study local galaxies" (Wyse 2006).

Ž. I. and B. S. acknowledge support by NSF grants AST 61-5991 and AST 07-07901 and by NSF grant AST 05-51161 to LSST for design and development activity. M. J. gratefully acknowledges support from the Taplin Fellowship and from NSF grant PHY-0503584. J. D. acknowledges NSF CAREER grant AST 02-38683. C. A. P. acknowledges support by NASA grants NAG5-13057 and NAG5-13147. T. C. B., Y. S. L., and T. S. acknowledge support from the US National Science Foundation under grants AST 04-06784 and AST 07-07776, as well as from grant PHY 02-16783, Physics Frontier Center/Joint Institute for Nuclear Astrophysics (JINA). P. R. F. acknowledges partial support through the Marie Curie Research Training Network ELSA (European Leadership in Space Astrometry) under contract MRTN-CT-2006-033481.

Funding for the SDSS and SDSS-II has been provided by the Alfred P. Sloan Foundation, the Participating Institutions, the
National Science Foundation, the US Department of Energy, the National Aeronautics and Space Administration, the Japanese Monbukagakusho, the Max Planck Society, and the Higher Education Funding Council for England. The SDSS Web Site is http://www.sdss.org.

The SDSS is managed by the Astrophysical Research Consortium for the Participating Institutions. The Participating Institutions are the American Museum of Natural History, Astrophysical Institute Potsdam, University of Basel, University of Cambridge, Case Western Reserve University, University of Chicago, Drexel University, Fermilab, the Institute for Advanced Study, the Japan Participation Group, Johns Hopkins University, the Joint Institute for Nuclear Astrophysics, the Kavli Institute for Particle Astrophysics and Cosmology, the Korean Scientist Group, the Chinese Academy of Sciences (LAMOST), Los Alamos National Laboratory, the Max-Planck-Institute for Astronomy (MPIA), the MaxPlanck-Institute for Astrophysics (MPA), New Mexico State University, Ohio State University, University of Pittsburgh, University of Portsmouth, Princeton University, the United States Naval Observatory, and the University of Washington.

\section{APPENDIX A}

\section{PHOTOMETRIC PARALLAX RELATION DERIVED USING GLOBULAR CLUSTERS}

In J08 we proposed a photometric parallax relation that did not explicitly use metallicity information, for two main reasons. First, the analysis included stars close to the faint limit of SDSS imaging for which the accuracy of photometric metallicity is significantly deteriorated due to increased $u$-band noise, and second, the sample also included red stars for which metallicity is difficult to estimate. The photometric parallax relation adopted in J08 implicitly takes metallicity effects into account by being somewhat shallower than a photometric parallax relation appropriate for a single-metallicity population: nearby stars ( $\lesssim 1 \mathrm{kpc}$ or so), which are predominantly red (due to the use of a flux-limited sample), have on average high disklike metallicities, while distant stars $(\sim 1-10 \mathrm{kpc})$ are predominantly blue stars with low metallicities (at a given $g-r$ or $g-i$ color, luminosity increases with metallicity for main-sequence stars). However, here we discuss only stars for which photometric metallicity estimates are available and, furthermore, they do not include very faint stars due to the flux limit $(u \lesssim 21)$ imposed by requiring proper-motion information. Hence, we can explicitly account for shifts of the photometric parallax relation as a function of metallicity.

The color-magnitude diagrams for globular clusters can be used to constrain the photometric parallax relation and its dependence on metallicity and to estimate systematic errors using the residuals between the adopted relation and individual clusters. For example, using three fiducial cluster sequences, $M_{V}(B-V)$, corresponding to metallicities, $[\mathrm{Fe} / \mathrm{H}]$, of $-2.20,-0.71$, and +0.12 , Beers et al. $(2000)$ spline interpolate between them to obtain $M_{V}$ for an arbitrary combination of $B-V$ and $[\mathrm{Fe} / \mathrm{H}]$. This is the method used to compute main-sequence distance estimates available from SDSS Data Release catalogs.

There are several reasons to revisit the method developed by Beers et al. (2000). First, a transformation from the Johnson system to SDSS system is required to apply their method to SDSS data. While this transformation is known to about 0.01 mag (Ivezić et al. 2007b), even such a small systematic error results in an uncertainty of absolute magnitude of $\sim 0.12$ mag for blue stars. Second, only three fiducial color-magnitude sequences are used, and it is not clear whether spline interpolation captures in detail the shift of the main sequence as a function of metallicity. Third, the impact of age variations on the assumed absolute magnitudes is not quantitatively known. Furthermore, it is not known how similar color-magnitude sequences are for different clusters with similar metallicity. It is, therefore, desirable to determine the photometric parallax relation using a larger number of clusters, with at least some of them observed by SDSS.

We use five globular clusters observed by SDSS, selected to have distance in the range 7-12 kpc (using distances from Harris 1996), to constrain the shape of the photometric parallax relation. This distance range ensures sufficient photometric quality for stars in the color range $g-i<0.8(g-r \lesssim 0.6)$, where photometric metallicity estimates are reliable. We augment this sample by data for six additional clusters compiled by VandenBerg \& Clem (2003), which significantly increase the sampled metallicity range and allow us to determine the shift of the photometric parallax relation as a function of metallicity. We use additional clusters observed by SDSS and by Clem et al. (2008), as well as constraints based on Hipparcos and ground-based trigonometric parallax measurements, to test our adopted photometric parallax relation.

\section{A1. METHODOLOGY AND RESULTS}

For clusters observed by SDSS, we select candidate cluster stars by limiting their angular distances from the cluster center to be less than the cluster radius determined by Simones et al. (2007). These radii, as well as distance and metallicity data from Harris (1996), are listed in Table 6. While the faint flux limits of SDSS imaging data limit this analysis only to relatively blue stars $(g-i<1.0)$, the color range where photometric metallicity can be determined is fully covered.

For each cluster, we determine the median $r$-band magnitude in 0.05 mag wide bins of the $g-i$ color. The red limit for the considered $g-i$ range is set by requiring $r<21.5$, and the blue end is selected to be at least 0.05 mag redder than the vertical part of the observed 
TABLE 6

The Globular Clusters Observed by SDSS and Used in the Photometric Parallax Analysis

\begin{tabular}{|c|c|c|c|c|c|c|c|c|}
\hline Name & $\begin{array}{c}D^{\mathrm{a}} \\
(\mathrm{kpc})\end{array}$ & $\begin{array}{c}R^{\mathrm{b}} \\
(\operatorname{arcmin})\end{array}$ & {$[\mathrm{Fe} / \mathrm{H}]_{\mathrm{H}}{ }^{\mathrm{c}}$} & {$[\mathrm{Fe} / \mathrm{H}]_{\mathrm{ph}}{ }^{\mathrm{d}}$} & $N^{\mathrm{e}}$ & $g i_{\min }{ }^{\mathrm{f}}$ & $g i_{\max }{ }^{\mathrm{g}}$ & $\begin{array}{c}\Delta r^{\mathrm{h}} \\
(\mathrm{mag})\end{array}$ \\
\hline 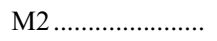 & 11.5 & 10.0 & -1.62 & -1.66 & 472 & 0.40 & 0.70 & 0.00 \\
\hline M3 & 10.4 & 17.5 & -1.57 & -1.41 & 1279 & 0.35 & 0.80 & 0.03 \\
\hline M5 ......................... & 7.5 & 17.5 & -1.27 & -1.27 & 1776 & 0.40 & 1.10 & -0.07 \\
\hline 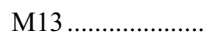 & 7.7 & 15.0 & -1.54 & -1.65 & 829 & 0.40 & 1.00 & 0.06 \\
\hline M15 ......................... & 10.3 & 12.5 & -2.26 & -2.09 & 676 & 0.30 & 0.70 & 0.01 \\
\hline
\end{tabular}

\footnotetext{
a Distance, taken from Harris (1996).

b Angular radius used for selecting cluster stars, taken from Simones et al. (2007).

c Metallicity, taken from Harris (1996).

d Median photometric metallicity for stars with $0.3<g-i<0.5$ and $u<21.5$.

e The number of stars used for estimating $[\mathrm{Fe} / \mathrm{H}]_{\mathrm{ph}}$ (errors are dominated by systematics).

${ }^{\mathrm{f}}$ The minimum $g-i$ color used in the analysis (determined by turnoff stars).

g The maximum $g-i$ color used in the analysis (determined from $r<21.5$ ).

h The median $r$-band offset for stars with $0.5<g-i<0.7$, relative to a prediction based on eqs. (A1)-(A3) (using distances listed in the second column).
}

sequences (turnoff stars). The red limit ensures sufficient signal-to-noise ratios, and the blue limit is designed to minimize the evolutionary (age) effects on the shape of the adopted relation. That is, we deliberately construct a relation that corresponds to small ages first and then study its variation with age using observed and model color-magnitude sequences. The adopted $g-i$ limits are listed in Table 6, and an example of this procedure (for M5) is shown in the top left panel of Figure 20.

We determine the shape of the photometric parallax relation by simultaneously fitting data for all five clusters. To do so, we first shift their $r$ versus $(g-i)$ sequences to a uniform (arbitrary) magnitude scale by requiring that the median $r$ magnitude for stars with $0.5<g-i<0.7$ is 0 . These offsets depend on the cluster metallicity, as discussed below. We then fit a parabola to all the data points, as a function of the $g-i$ color, using an unweighted least-squares method (a third-order polynomial is unnecessary to within $\sim 0.05$ mag). We used the $g-i$ color because it has better signal-to-noise ratio properties than $g-r$ and $r-i$ colors. We did not use the so-called projection on stellar locus technique developed in J08 because it produces essentially identical results for relatively bright stars considered here. The stellar locus parameterization from J08 can be used to express the fiducial sequence in terms of the $g-r$ and $r-i$ colors, if needed.

The best-fit fiducial sequence is

$$
M_{r}^{0}(g-i)=-2.85+6.29(g-i)-2.30(g-i)^{2}
$$

with $M_{r}^{0}=r-\langle r\rangle=M_{r}-\left\langle M_{r}\right\rangle$, valid for $0.3<(g-i) \lesssim 1.0$ and the medians evaluated in the $0.5<g-i<0.7$ color range. As discernible from the cluster data shown in the top right panel of Figure 20, individual clusters follow the mean relation to within $0.1 \mathrm{mag}$ or better (the rms scatter for all data points around the best-fit relation is $0.08 \mathrm{mag}$ ). We compare the slopes of the predicted and observed sequences using the difference in absolute magnitudes at $g-i=0.4$ and at $g-i=0.7$ (the predicted value is 1.25 mag). The largest discrepancies of $\sim 0.1 \mathrm{mag}$ are observed for M13 (the observed sequence is steeper) and M15 (the observed sequence is shallower). These discrepancies may be caused by a combination of metallicity and age effects.

We proceed by assuming that the shape of the color-magnitude sequence given by equation (A1) is a universal function independent of metallicity, and that its normalization depends only on metallicity. While this is not strictly true, as we discuss below, the available data are not sufficient to robustly constrain the shape variation as a function of metallicity (and possibly other parameters, e.g., helium content; see Demarque \& McClure 1980).

We place the color-magnitude sequences for each cluster on an absolute scale using distances from Harris (1996). The offset of the measured globular cluster sequences relative to the best-fit fiducial sequence is a strong function of metallicity. We improve observational constraints on this relation by considering six additional clusters discussed by VandenBerg \& Clem (2003). We used their figures to estimate for each cluster its $M_{V}$ at $B-V=0.60$ (corresponding to $g-i=0.57$ ), listed in Table 7 . The corresponding $M_{r}$ (i.e., the $V-r$ color) are computed using the SDSS-to-Johnson system transformations from Ivezić et al. (2007a).

The data shown in the bottom left panel of Figure 20 strongly suggest a nonlinear relationship (without the extended metallicity baseline thanks to the VandenBerg \& Clem [2003] data, the five SDSS clusters would imply a linear relationship). The best-fit parabola is

$$
\Delta M_{r}([\mathrm{Fe} / \mathrm{H}])=4.50-1.11[\mathrm{Fe} / \mathrm{H}]-0.18[\mathrm{Fe} / \mathrm{H}]^{2}
$$

where $\Delta M_{r}$ is defined by

$$
M_{r}(g-i,[\mathrm{Fe} / \mathrm{H}])=M_{r}^{0}(g-i)+\Delta M_{r}([\mathrm{Fe} / \mathrm{H}])
$$

The rms scatter around the best-fit relation is $0.05 \mathrm{mag}$ for the 11 clusters used in the fit, with the maximum deviation of 0.08 mag. This remarkably small scatter around a smooth best-fit function suggests that the determination of $\Delta M_{r}([\mathrm{Fe} / \mathrm{H}])$ offsets for individual 
M5 in SDSS
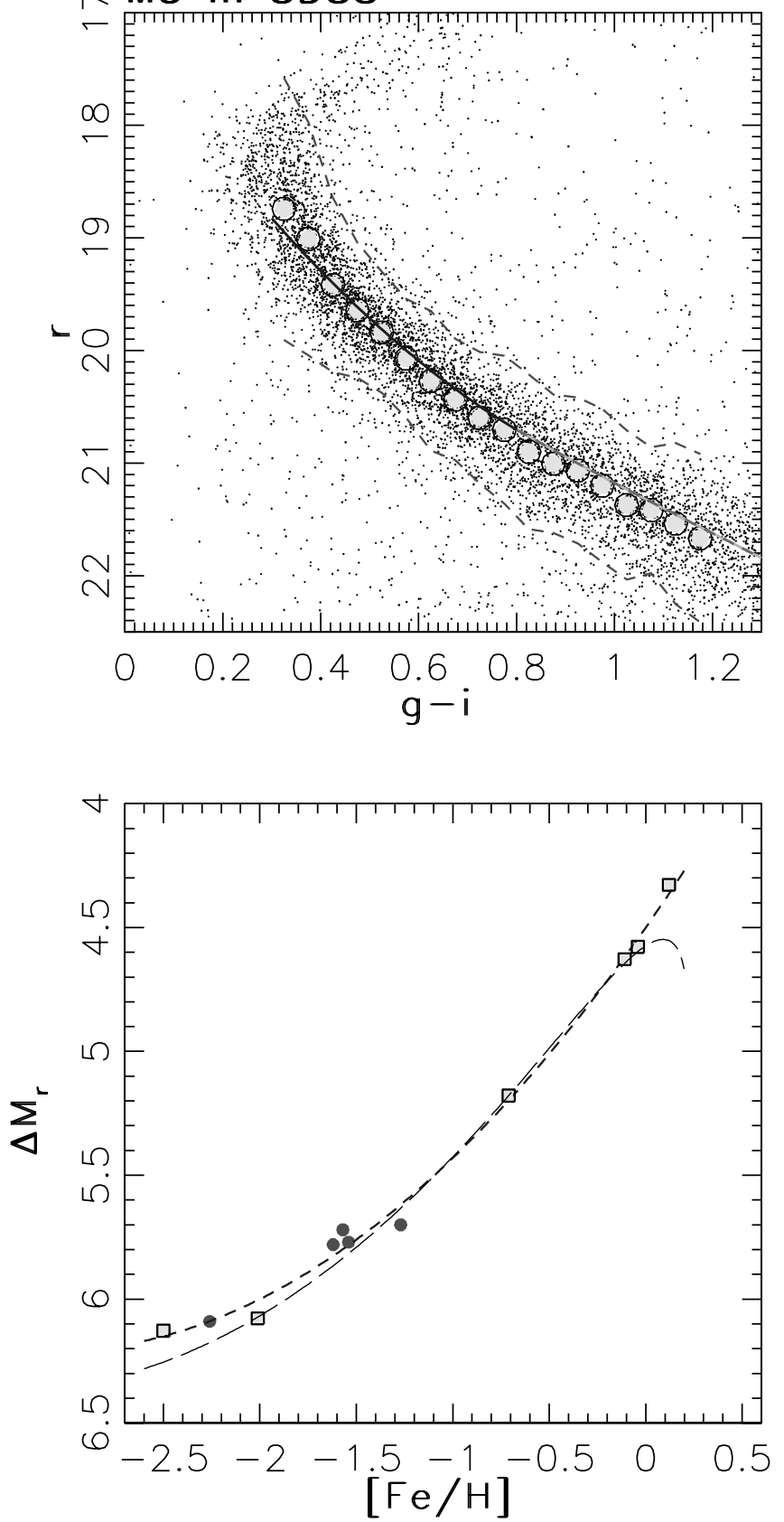
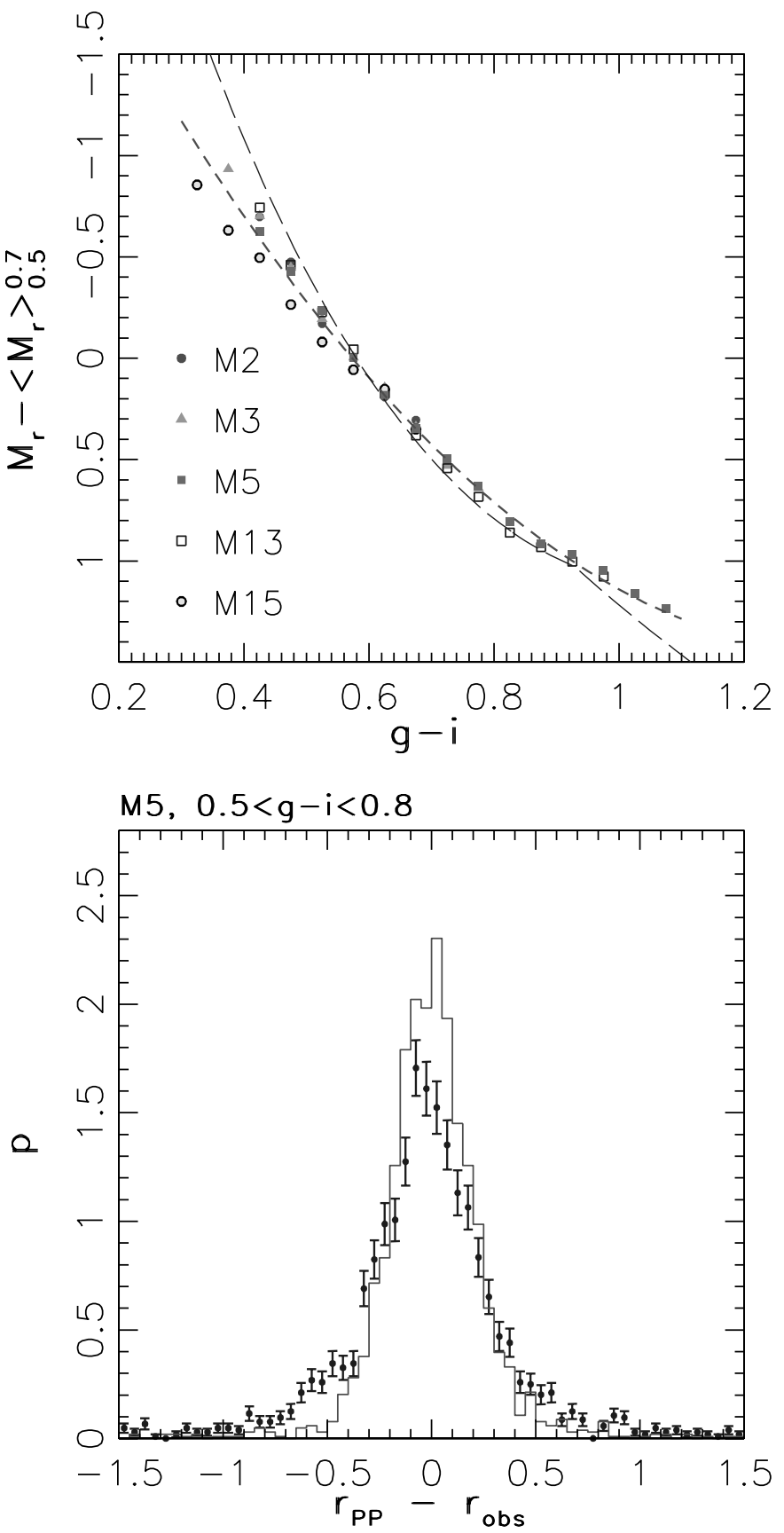

FIG. 20.-Top left panel: Color-magnitude diagram for the globular cluster M5 measured by SDSS. Individual stars are displayed as small dots, while the large circles show binned medians. The two dashed lines show the $2 \sigma$ envelope around these medians, and the solid line is the prediction based on the adopted photometric parallax relation (see text). The top right panel shows analogous binned medians for five globular clusters, with each sequence rescaled by the median magnitude for stars with $0.5<g-i<0.7$. The short-dashed line shows a best-fit fiducial sequence (eq. [A1]). For a comparison, the long-dashed line shows the [Fe/H] $=-2.20$ fiducial sequence from Beers et al. (2000). The filled circles in the bottom left panel show the absolute magnitude offsets relative to the fiducial relation for the five globular clusters listed in Table 6 . The squares show analogous offsets for the six globular clusters listed in Table 7. The short-dashed line is the best unweighted linear fit to both data sets (eq. [A2]). The thin long-dashed line is the $\Delta M_{V}$ vs. [Fe/H] relationship from Laird et al. (1988), shifted to produce the same $\Delta M_{r}$ at $[\mathrm{Fe} / \mathrm{H}]=-1.0$ as the best fit derived here. The symbols with error bars (representing counting noise) in the bottom right panel show the distribution of differences between $r$-band magnitudes predicted using the adopted photometric parallax relation and the observed values. The histogram shows the expected scatter due to photometric errors. [See the electronic edition of the Journal for a color version of this figure.]

clusters has a similar precision. Note, however, that the overall scale of $M_{r}(g-i,[\mathrm{Fe} / \mathrm{H}])$ includes all systematic errors inherent in cluster distances that are adopted from the Harris (1996) compilation (including a possible covariance with cluster metallicity). The adopted relation produces gradients of $d M_{r} / d[\mathrm{Fe} / \mathrm{H}]=-0.57 \mathrm{mag} \mathrm{dex}^{-1}$ at the median halo metallicity $([\mathrm{Fe} / \mathrm{H}]=-1.50)$ and $-1.0 \mathrm{mag} \mathrm{dex}{ }^{-1}$ at the median thin-disk metallicity $([\mathrm{Fe} / \mathrm{H}]=-0.2)$, with an offset of $1.05 \mathrm{mag}$ between these two [Fe/ $\left.\mathrm{H}\right]$ values. As illustrated in the bottom left panel of Figure 20 , the best-fit relation derived here is in excellent agreement at $[\mathrm{Fe} / \mathrm{H}]<0$ with an analogous relation proposed by Laird et al. (1988).

The distributions of differences between the $r$-band magnitudes predicted using the above expressions and the observed values for individual stars are consistent with expected noise due to photometric errors for all five clusters (see the bottom right panel of Fig. 20 for 
TABLE 7

Additional Cluster Data from VandenBerg \& Clem (2003)

\begin{tabular}{|c|c|c|c|}
\hline Name & {$[\mathrm{Fe} / \mathrm{H}]^{\mathrm{a}}$} & $M_{V}^{\mathrm{b}}$ & $M_{V}{ }^{\mathrm{c}}$ \\
\hline M92 ............ & -2.50 & 6.30 & 6.32 \\
\hline 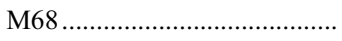 & -2.01 & 6.25 & 6.18 \\
\hline 47 Tuc ......................... & -0.71 & 5.35 & 5.37 \\
\hline Pleiades ........................ & -0.11 & 4.80 & 4.79 \\
\hline 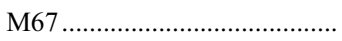 & -0.04 & 4.75 & 4.72 \\
\hline 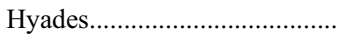 & +0.12 & 4.50 & 4.53 \\
\hline
\end{tabular}

a Metallicity, taken from VandenBerg \& Clem (2003), except for 47 Tuc, which is taken from Beers et al. (2000) (VandenBerg \& Clem [2003] adopted $[\mathrm{Fe} / \mathrm{H}]=-0.83$, which produces a $0.1 \mathrm{mag}$ fainter $M_{V}$ prediction).

b The absolute $V$-band magnitude for $B-V=0.60$, determined with an accuracy of $0.05-0.10 \mathrm{mag}$, from figures presented in VandenBerg $\&$ Clem (2003).

${ }^{c}$ The absolute $V$-band magnitude for $B-V=0.60$, determined using eqs. (A1)-(A3) and the SDSS-to-Johnson transformations from Ivezić et al. (2007b).

an example based on M5). At the faint end ( $r \sim 21)$, the expected uncertainty in $M_{r}$ is about 0.3 mag (random error per star) and is dominated by random photometric errors in the $g-i$ color. At the bright end, the $g-i$ errors $(\sim 0.03 \mathrm{mag})$ contribute an $M_{r}$ uncertainty of $\sim 0.15 \mathrm{mag}$, and an error in $[\mathrm{Fe} / \mathrm{H}]$ of 0.1 dex results in an $M_{r}$ error of $\lesssim 0.1 \mathrm{mag}$. The random errors in the $g-i$ color and photometric metallicity are by and large uncorrelated because the $u$-band errors dominate the latter.

The SDSS cluster data discussed here are not sufficient to extend the fiducial sequence beyond $g-i \sim 1$. While not required for the analysis presented here, we extend for completeness the adopted relation using the shape of the "bright" relation from J08. Expressed as a function of the $g-i$ color,

$$
M_{r}^{0}(g-i)=-1.93+4.39(g-i)-1.73(g-i)^{2}+0.452(g-i)^{3},
$$

valid for $(g-i)>0.8$. We test this extension further below.

\section{A2. TESTING}

Using SDSS observations for five clusters listed in Table 6, we first determined the median photometric metallicity for each cluster, using the best-fit expressions derived in this work. To avoid contamination by disk stars and noisy metallicity estimates, we only use stars with $0.3<g-i<0.5$ and $u<21.5$. Remarkably, the photometric metallicity estimates are consistent with the values taken from Harris (1996) to within $\sim 0.1$ dex. This test ensures that equation (A2) can also be used with photometric metallicity estimates.

We have tested equations (A1)-(A3) using an independent sample of clusters observed by SDSS at distances beyond our cutoff of $12 \mathrm{kpc}$ (NGC 4147, NGC 5053, NGC 5466, NGC 5024, and Pal 5). The first four clusters have low metallicities ([Fe/H] $\sim-2.0$ ), and for Pal $5[\mathrm{Fe} / \mathrm{H}]=-1.41$. The $r$ versus $g-i$ ridgelines predicted by equation (A1) agree well with the observed sequences (the data are much noisier than for the first five nearer clusters due to their fainter apparent magnitudes). The only significant discrepancy is observed for Pal 5, for which the predicted magnitudes are too faint by $\sim 0.5 \mathrm{mag}$ (using a distance of $23.2 \mathrm{kpc}$ ).

To test the extension of the photometric parallax relation to red colors, we use the $M_{V}(B-V)$ sequence for $\mathrm{M}$ dwarfs with the Hipparcos data, as compiled in Figure 17 from VandenBerg \& Clem (2003): for $B-V=(1.2,1.3,1.4)$, corresponding to $g-i=$ $(1.51,1.70,1.93)$, we adopt $M_{V}=(7.5,8.0,8.5)$. Assuming that the metallicity of those stars is equal to the median thin-disk metallicity, $[\mathrm{Fe} / \mathrm{H}]=-0.13$ (Nordström et al. 2004; Allende Prieto et al. 2004), we obtain $M_{V}=(7.42,7.91,8.54)$. For the reddest data point with $V-I=2.0, M_{V}=9.5$, and we obtain $M_{V}=9.47$. This excellent agreement suggests that the extension given by equation (A4) is good to within $\sim 0.1$ mag for $g-i<2.2$.

For redder colors $(g-i>2.0$ ), we compared our results with the relation derived by J. Bochanski et al. (2008, in preparation), which is based on ground-based trigonometric parallaxes for nearby stars (D. Golimowski et al. 2008, in preparation). Assuming a median metallicity of $[\mathrm{Fe} / \mathrm{H}]=-0.13$ for these stars, we found that the performance of equation (A4) starts deteriorating around $g-i=3.0$. In the range $2.0<g-i<2.8$, our relation agrees with the J. Bochanski et al. (2008, in preparation) relation within 0.07 mag (rms) and $\sim 0.03 \mathrm{mag}$ (median), and maximum deviation $<0.1 \mathrm{mag}$, evaluated on a grid with $0.01 \mathrm{mag}$ steps. A linear relation in the range $2.8<g-i<4.0$,

$$
M_{r}^{0}(g-i)=-4.40+3.97(g-i),
$$

is a much better approximation to the observed sequence than equation (A4) (but for a detailed fit please consult J. Bochanski et al. 2008, in preparation). Note that for $[\mathrm{Fe} / \mathrm{H}]=-0.13$, this relation must be shifted by 4.64 mag to obtain $M_{r}$ (see eq. [A2]).

As an additional test of the relation derived here, we compare it to color-magnitude sequences measured by Clem et al. (2008) for three clusters that have turnoff colors bluer than $g-i=0.6$ (M3, M13, and M92). Their data were obtained in the SDSS "prime" 

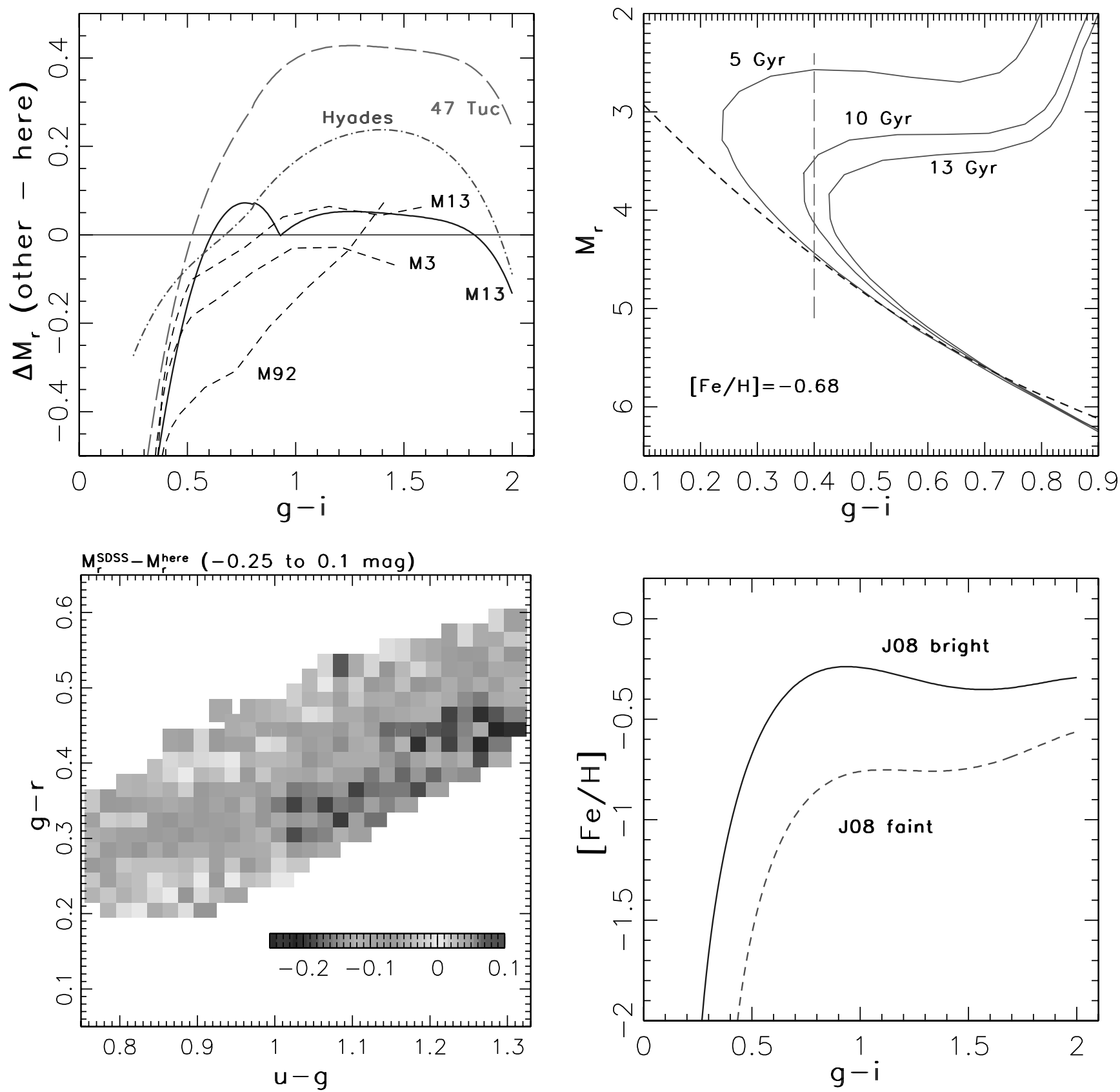

FIg. 21.-Top left panel: Difference between the color-magnitude sequences from Beers et al. (2000) for three metallicity values $($ solid line: $[\mathrm{Fe} / \mathrm{H}]=-2.20 ;$ longdashed line: $[\mathrm{Fe} / \mathrm{H}]=-0.71$; dot-dashed line: $[\mathrm{Fe} / \mathrm{H}]=+0.12)$ and eqs. $(\mathrm{A} 1)-(\mathrm{A} 3)$ derived here. The three short-dashed lines show analogous differences for the M3, M13, and M92 sequences from Clem et al. (2008), as marked. The systematic differences for blue stars are due to age effects. The solid lines in the top right panel show $M_{r}$ for a Girardi et al. (2004) model with [Fe/H] $=-0.68$, evaluated for three ages, as marked. The models are offset by 0.2 mag to brighter magnitudes, to match $M_{r}$. computed using eqs. (A1) and (A7) (shown as the dashed line). The vertical long-dashed line marks the turnoff color for disk stars. The bottom left panel shows the median differences between the SDSS distance modulus for main-sequence stars (determined using the Beers et al. [2000] sequences) and the values estimated using eqs. (A1) and (A7), with gray scale as shown in the inset. The two methods agree at the $\sim 0.1$ mag level (the mean for the median difference per pixel; the distribution rms width is $\sim 0.2 \mathrm{mag}$ ). The bottom right panel shows implied metallicity, estimated using eqs. (A1) and (A7), for the two photometric parallax relations proposed by J08 (solid line: "bright" relation; dashed line: "faint" relation). At the blue end, they bracket the median halo metallicity $([\mathrm{Fe} / \mathrm{H}]=-1.50)$; at the red end they sample the thin/thick-disk metallicity range. [See the electronic edition of the Journal for a color version of this figure.]

system, and we used expressions from Tucker et al. (2006) to transform those sequences onto the SDSS native system. For $g-i>0.5$, their sequences for M3 and M13 are in good agreement $(<0.2 \mathrm{mag})$ with our predictions, while for blue colors close to the turnoff color, they become progressively brighter, as expected (see the top left panel of Fig. 21). For M92, discrepancies are larger than $\sim 0.2$ mag even for red colors $(g-i \sim 1)$. However, based on photometric transformations from Tucker et al. (2006) and Ivezić et al. (2007a), we find that the M92 sequence in the SDSS "prime" system from Clem et al. (2008) and the M92 sequence in the Johnson system from VandenBerg \& Clem (2003) are not consistent. For example, $V=20.9$ at $B-V=0.6$ taken from VandenBerg \& Clem (2003) implies $r=20.7$, while data listed in Table 3 from Clem et al. (2008) imply $r=20.45$ at the corresponding color. We emphasize that the same photometric transformations result in good agreement for the other two clusters, and that the color-magnitude sequence for M92 from VandenBerg \& Clem (2003) agrees with our relation to within $0.1 \mathrm{mag}$. 
The top left panel of Figure 21 shows a comparison of the relation derived here with the three sequences from Beers et al. (2000). Similarly to the comparison with the Clem et al. (2008) sequences, our relation predicts fainter magnitudes for blue turnoff stars, as expected. We emphasize that these differences are not due to errors in the color-magnitude sequences adopted by Beers et al. (2000) because they agree with other sources, e.g., with the VandenBerg \& Clem (2003) data. Rather, the differences are due to our design choice to exclude from fitting the parts of the clusters' color-magnitude sequences that are too close to their turnoff color.

Our results show that the Beers et al. (2000) spline interpolation of metallicity effects based on only three clusters performs remarkably well. The largest overall discrepancy between our photometric parallax relation and the three Beers et al. (2000) sequences for red colors $(g-i>0.6)$ is observed for 47 Tuc: for $1.0<g-i<1.8$, the predicted $M_{r}$ are too bright by 0.4 mag. Since agreement at our fiducial $g-i \sim 0.6$ is satisfactory, this difference implies that the color-magnitude sequence for 47 Tuc is steeper than for other clusters discussed here. This peculiarity of 47 Tuc has been known for some time and may be related to its anomalous helium content (Demarque \& McClure 1980; Hesser et al. 1987). We note that our relation predicts absolute magnitudes for red stars $(B-V>1)$ that are brighter by $\sim 0.3 \mathrm{mag}$ than the data for the extremely metal-rich $([\mathrm{Fe} / \mathrm{H}]=+0.37)$ open cluster NGC 6791 from VandenBerg \& Clem (2003).

\section{A3. AGE EFFECTS AND COMPARISON WITH MODELS}

By design, the photometric parallax relation derived here avoids the increased curvature of the color-magnitude sequence close to the turnoff color. Its blue edge is constrained by the parts of the M3 and M15 sequences that are redward from their turnoff colors (see Table 6 and the top right panel of Fig. 20). For stars with turnoff colors, the predicted absolute magnitudes can be up to $\sim 1$ mag too faint. For example, for M5 turnoff stars selected by $0.25<g-i<0.35(\langle r\rangle=18.6)$, the difference between predicted and observed $r$-band magnitudes is well described by a Gaussian distribution with a mean of 0.22 mag and $\sigma=0.49$ mag, implying underestimated distances by $11 \%$, on average.

The effect of age on turnoff color and absolute magnitude, as a function of metallicity, can be gauged with the aid of model isochrones, e.g., such as those developed for the SDSS photometric system by Girardi et al. (2004). While modeling difficulties prevent absolute normalization of such models to better than $\sim 0.1-0.2$ mag even for hot stars (and much worse for stars with $g-i>1$ ), their relative behavior, as a function of age, provides valuable guidance. The Girardi et al. (2004) models show that the turnoff color is bluer than $g-i=0.6$ even for 13 Gyr old populations and a metallicity at the upper end of the range relevant here $([\mathrm{Fe} / \mathrm{H}]=-0.4)$. Hence, the adopted relation is insensitive to age effects for $g-i>0.6$. For $g-i<0.6$, it needs to be corrected as a function of metallicity and age.

The mean ages of halo and disk stars considered in this work can be estimated from the blue edge of their color distributions. The number of stars drops precipitously bluer than $g-i \sim 0.25$ for the low-metallicity subsample (Fe/ $\mathrm{H} \lesssim-1$, halo stars), and at $g-i \sim$ 0.4 for the high-metallicity subsample (disk stars). Interestingly, the Girardi et al. (2004) models suggest similar age for both subsamples: $\sim 10 \mathrm{Gyr}$, with an estimated uncertainty of $\sim 2 \mathrm{Gyr}$ (due to metallicity and color zero-point uncertainties; we adopted 0.2 dex and $0.05 \mathrm{mag}$, respectively). Motivated by this result, we derive an age correction appropriate for stars with median halo metallicity and age of $\sim 10$ Gyr using the color-magnitude sequence for cluster M13 ([Fe/ $\mathrm{H}]=-1.54)$. For $0.22<g-i<0.58$

$$
\Delta M_{r}^{\mathrm{M} 13}(g-i)=-2.17+6.64(g-i)-5.00(g-i)^{2},
$$

which increases from 0 at the red edge to $-0.95 \mathrm{mag}$ at $g-i=0.22$ and has to be added to the right-hand side of equation (A3).

This correction for age is not strictly applicable to stars with higher disklike metallicity. However, the Girardi et al. (2004) models suggest that the error is small, $<0.2 \mathrm{mag}$ for $g-i>0.45$ (i.e., 0.05 redder than the turnoff color for disk stars), as illustrated in the top right panel of Figure 21. For this reason, we adopt equation (A6) as a universal age correction for stars bluer than $g-i<0.58$.

Given different expressions for three color ranges (eqs. [A1], [A4], and [A5]) and the above age correction, for convenience we fit a fifth-order polynomial to a vector of $M_{r}$ values generated using the appropriate expressions for $0.2<g-i<4.0$, with a step size of 0.01 mag. Our final expression

$$
M_{r}^{0}(g-i)=-5.06+14.32 x-12.97 x^{2}+6.127 x^{3}-1.267 x^{4}+0.0967 x^{5},
$$

where $x=(g-i)$, reproduces individual $M_{r}$ values with an rms of 0.05 mag and maximum deviation below 0.1 mag. Together with equations (A2) and (A3), this is the final photometric parallax relation used in this work.

We have compared a large number of Girardi et al. (2004) models that span the relevant range of metallicities $(-2.3<[\mathrm{Fe} / \mathrm{H}]<0)$ and ages $(1-13 \mathrm{Gyr}$ ) with the resulting photometric parallax relation. Model predictions are in good agreement (an rms of $\sim 0.1 \mathrm{mag}$ ) with the $M_{r}$ versus [Fe/H] dependence described by equation (A1), but the model $M_{r}$ predictions are systematically too faint by $\sim 0.2$ mag (evaluated at $g-i=0.7$ ). Possible explanations for this difference are that (1) the model stars are too small by $\sim 10 \%$, (2) the model $g-i$ color is too red by $0.06 \mathrm{mag}$, and (3) the model $[\mathrm{Fe} / \mathrm{H}]$ scale is offset relative to the SDSS scale by $\sim 0.3$ dex to larger values. A plausible combination of these effects, e.g., an error of $3 \%$ in sizes, 0.02 mag in color, and 0.1 dex in metallicity, brings data and models into agreement (the probability that all three effects would have the same sign is $12 \%$ ).

\section{A4. COMPARISON WITH SDSS DISTANCES AND J08}

With the adopted age correction (eq. [A6]), our final expression is expected to produce very similar distances to those published in SDSS Data Release catalogs for blue stars $(g-i<2)$. We have confirmed that this is the case: the median offset of implied $M_{r}$ evaluated in small bins of $u-g$ and $g-r$ color (see the bottom left panel of Fig. 21) is $-0.07 \mathrm{mag}$, with an rms of $0.06 \mathrm{mag}$. These differences are smaller than the intrinsic errors of the photometric parallax method $(\sim 0.1-0.2 \mathrm{mag})$. 
Using equations (A2), (A3), and (A7), we can now determine "effective" metallicity that the two photometric parallax relations proposed in $\mathrm{J} 08$ correspond to, as a function of the $g-i$ color (see the bottom right panel of Fig. 21). As designed, those two relations bracket the median halo metallicity $([\mathrm{Fe} / \mathrm{H}]=-1.50)$ at the blue end and sample the thin/thick-disk metallicity range at the red end.

In summary, the relations proposed here are in good agreement $(<0.1 \mathrm{mag})$ with the clusters M3 and M13 at the low-metallicity end for $g-i<1.5$, and with local stars with trigonometric parallaxes for $g-i>1.5$. At a fiducial color $g-i=0.6$, in the middle of the color range where photometric metallicity can be estimated, the rms scatter around the best-fit $\Delta M_{r}$ versus $[\mathrm{Fe} / \mathrm{H}]$ curve is 0.08 mag. Even in cases of known peculiar behavior (e.g., 47 Tuc) and at the high-metallicity end (e.g., NGC 6791), discrepancies do not exceed 0.4 mag. Compared to the Beers et al. (2000) relations used by the SDSS, here we provide an estimate of the scatter around mean relations, a closedform expression for the metallicity dependence, and extend the method's applicability farther into the red, to $g-i \sim 4$. Given the larger number of globular clusters observed in the SDSS system used here, as well as tests based on external data sets, it is likely that distance estimates for main-sequence stars based on the photometric parallax method (using both relations derived here and the Beers et al. [2000] relations) do not suffer from systematic errors larger than $\sim 10 \%$. While these systematic distance errors are not overwhelming, they could, in principle, have an impact on the analysis of the Milky Way kinematics. We discuss such issues further in Paper III (B08).

\section{APPENDIX B}

\section{ADDITIONAL DISCUSSION OF THE PHOTOMETRIC TEMPERATURE ESTIMATOR}

Often, the inverse of the effective temperature is fitted as a linear function of color (e.g., CPF06). The best fit

$$
\frac{5040 \mathrm{~K}}{T_{\text {eff }}}=0.532(g-r)+0.654
$$

results in the same systematic errors and rms scatter as equation (3), with the largest difference between the two relations below $50 \mathrm{~K}$.

A lower limit for the errors in estimation of the photometric effective temperature can be readily computed using equation (3) and the photometric errors in the $g-r$ color (the median value is $0.025 \mathrm{mag}$, and $0.03 \mathrm{mag}$ at $g=19.5$; these values, computed by the photometric pipeline, are reliable, as discussed in detail by Sesar et al. 2007). This is a lower limit because the contribution of errors in the spectroscopic effective temperature is not included. The standard deviation for the distribution of metallicity residuals normalized by these errors is 1.2. Hence, one is tempted to conclude that the accuracy of the effective temperature estimator is limited by the SDSS photometric errors. However, this conclusion is not consistent with the behavior of the $\log T_{\text {eff }}$ versus $g-r$ relation for a subset of 13,719 stars for which more accurate photometry, based on $\sim 10$ repeated SDSS observations, is available (Ivezić et al. 2007a). Although for these stars the median error in the $g-r$ color is only $0.008 \mathrm{mag}$, the standard deviation for $\log T_{\text {eff }}$ residuals is not appreciably smaller (the expectation is a decrease by a factor of 3 ). Therefore, it is quite likely that the contribution of errors in the spectroscopic effective temperature to the scatter of $\log T_{\text {eff }}$ residuals is not negligible. Indeed, the implied value of $\sim 100 \mathrm{~K}$ agrees well with an independent estimate based on a comparison to high-resolution spectral data, as discussed by Beers et al. (2006). The analyzed color range spans about $15 \mathrm{MK}$ spectral subtypes (from $\sim F 5$ to $\sim$ G9/K0; Bailer-Jones et al. 1997, 1998). Hence, the uncertainty in the photometric effective temperature estimate of $100 \mathrm{~K}$ corresponds to about one spectral subtype, or equivalently, an error of one spectral subtype corresponds to a $g-r$ error of $0.02 \mathrm{mag}$.

A good correlation between the spectroscopic effective temperature and $g-r$ color extends beyond the restricted color range where the photometric metallicity method is applicable $(0.2<g-r<0.4)$. We find that everywhere in the $-0.3<g-r<1.3$ color range (roughly $-0.1<B-V<1.3$ ), the relation

$$
\log \left(T_{\text {eff }} / \mathrm{K}\right)=3.882-0.316(g-r)+0.0488(g-r)^{2}+0.0283(g-r)^{3}
$$

achieves systematic errors below 0.004 dex and overall rms of 0.008 dex. The corresponding temperature range is $4000-10,000 \mathrm{~K}$. When the residuals are binned in 0.1 dex wide bins of metallicity and $\log g$, the largest median residual is 0.006 dex. Equation (B1) remains valid in the $-0.3<g-r<0.8$ range but also requires nonlinear terms if extended to redder colors (or a different linear fit for the $0.8<g-r<1.3$ range).

Due to the expanded $g-r$ range, the impact of metallicity and $\log g$ on $\log T_{\text {eff }}$ residuals is expected to be larger for this relation than for equation (3). Using Kurucz (1979) models, we find that the strongest dependence on metallicity is expected in the $0.4<g-r<1.2$ color range, with a gradient of $\sim 0.015 \mathrm{dex} \mathrm{dex}^{-1}$ (for a related discussion see also Lenz et al. 1998). The measured value for the SDSS sample is $0.012 \mathrm{dex} \mathrm{dex}^{-1}$ and implies up to $\sim 200 \mathrm{~K}$ offsets as metallicity varies from -2.0 to -0.5 . The strongest dependence on log $g$ is expected in the $-0.2<g-r<0.1$ color range, with a gradient of $0.02 \mathrm{dex} \mathrm{dex}^{-1}$. The measured value for the SDSS sample is $\sim 0.004 \mathrm{dex} \mathrm{dex}^{-1}$, or about 5 times smaller $(150 \mathrm{~K}$ vs. $720 \mathrm{~K}$ variation, as $\log g$ varies by 2 dex at $g-r=0)$. We do not understand the cause of this discrepancy.

\section{APPENDIX C}

\section{ADDITIONAL DISCUSSION OF THE PHOTOMETRIC METALLICITY ESTIMATOR}

In two restricted color regions, simpler expressions than equation (4) can suffice. In a low-metallicity region defined by $0.8<u-$ $g<1.0$ (and $0.2<g-r<0.6$, of course), the relation

$$
[\mathrm{Fe} / \mathrm{H}]_{\mathrm{ph}}=5.14(u-g)-6.10
$$


reproduces the spectroscopic metallicity of about 27,000 stars with an rms scatter of 0.29 dex. We note that this is essentially the same expression as obtained by Ivezić et al. (2006), using a preliminary version of the spectroscopic parameter pipeline, except for an overall shift in metallicity by $0.2 \mathrm{dex}$. This shift is due to the fact that the SDSS stellar parameter pipeline was still under development when the analysis of Ivezić et al. (2006) was performed.

In the high effective temperature region $\left(5800 \mathrm{~K}<T_{\text {eff }}<6600 \mathrm{~K}\right)$ defined by $0.2<g-r<0.4$, the relation

$$
[\mathrm{Fe} / \mathrm{H}]_{\mathrm{ph}}=-21.88+47.39(u-g)-35.50(u-g)^{2}+9.018(u-g)^{3}
$$

reproduces spectroscopic metallicity of about 34,000 stars with an rms scatter of 0.30 dex. In the range $0.8<u-g<1.4$ (corresponding to $-2.0<[\mathrm{Fe} / \mathrm{H}]<-0.4$ ) systematic errors do not exceed 0.1 dex. The systematic errors are larger than for equation (4) because the lines of constant metallicity in the $g-r$ versus $u-g$ diagram are not exactly vertical. Despite having somewhat poorer performance, equations $(\mathrm{C} 1)$ and $(\mathrm{C} 2)$ are convenient when estimating the impact of $u-g$ color error on photometric metallicity error. An error in the $u-g$ color of $0.02 \mathrm{mag}$ (typical of both systematic calibration errors and random errors at the bright end for SDSS data) induces an error in $[\mathrm{Fe} / \mathrm{H}]$ that varies from 0.02 dex at $[\mathrm{Fe} / \mathrm{H}]=-0.5(u-g=1.28)$ to 0.11 dex at $[\mathrm{Fe} / \mathrm{H}]=-1.5(u-g=0.89)$. At $g=19.5$, the median $u-g$ error for single-epoch SDSS data is 0.06 mag for point sources with $0.2<g-r<0.4$, corresponding to median random metallicity errors of 0.10 dex for disk stars and 0.30 dex for halo stars (for a detailed dependence of SDSS random photometric errors on magnitude, see Sesar et al. 2007).

The metallicity versus $u-g$ relation has a smaller slope at the red end (both eqs. [4] and [C2]) and effectively introduces an upper limit on estimated metallicity. For example, for $u-g=1.3$ and $g-r=0.4,[\mathrm{Fe} / \mathrm{H}]_{\mathrm{ph}}=-0.44$ (from eq. [4], and -0.46 using eq. [C2]). Such an upper limit is in agreement with the data analyzed here, but we emphasize that the data set under consideration does not include significant numbers of stars with higher metallicity. Such stars are presumably nearby thin-disk stars, which in the $0.2<g-r<0.6$ range are typically saturated in SDSS data (most SDSS data to date are obtained at high Galactic latitudes). It is thus possible that metallicity estimates given by both equations (4) and (C2) would be biased toward lower values for stars with $[\mathrm{Fe} / \mathrm{H}]>-0.5$, resulting in a "metallicity compression." Some evidence that this is a detectable but not a major effect is discussed in $\S 3.4 .1$ and in more detail by Lee et al. (2007b). It will be possible to quantify this effect in detail using the data for metal-rich stars from the ongoing SDSS spectroscopic survey of low Galactic latitudes (SEGUE).

Given that the $u$-band photometric errors limit the precision of photometric metallicity estimates at the faint end, it is prudent to test whether the position of a star in the $r-i$ versus $g-r$ color-color diagram could be used as an alternative method. We selected subsamples of stars in 0.02 mag wide $g-r$ bins and inspected the dependence of spectroscopic metallicity on the $r-i$ color in the range $-2.5<[\mathrm{Fe} / \mathrm{H}]<-0.5$. The strongest correlation between $[\mathrm{Fe} / \mathrm{H}]$ and $r-i$ color is observed around $g-r \sim 0.4$, with a gradient of $\Delta(r-i) / \Delta[\mathrm{Fe} / \mathrm{H}] \sim 0.017 \mathrm{mag} \mathrm{dex}^{-1}$. Hence, the effect of metallicity on the $r-i$ color is about 10 times smaller than for the $u-$ $g$ color. With the $r-i$ color kept fixed, we find $\Delta(g-r) / \Delta[\mathrm{Fe} / \mathrm{H}] \sim 0.04 \mathrm{mag} \mathrm{dex}^{-1}$. When using only the gri bands, the photometric metallicity errors are about 0.3 dex at the bright end and 0.5 dex at $g=19.5(<0.1$ dex and $<0.3$ dex for $u g r$-based estimates). Therefore, the best approach for estimating photometric metallicity using SDSS data is to use the $u g r$ bands.

Abadi, M. G., Navarro, J. F., Steinmetz, M., \& Eke, V. R. 2003, ApJ, 597, 21

Abazajian, K., et al. 2003, AJ, 126, 2081

. 2004, AJ, 128, 502

2005, AJ, 129, 1755

Adelman-McCarthy, J. K., et al. 2006, ApJS, 162, 38 2008, ApJS, 175, 297

Allende Prieto, C., Barklem, P. S., Lambert, D. L., \& Cunha, K. 2004, A\&A, 420,183

Allende Prieto, C., et al. 2006, ApJ, 636, 804 2007, AJ, submitted (arXiv:0710.5780)

Bahcall, J. N., \& Soneira, R. M. 1980, ApJS, 44, 73

Bailer-Jones, C. A. L., Irwin, M., Gilmore, G., \& von Hippel, T. 1997, MNRAS, 292, 157

Bailer-Jones, C. A. L., Irwin, M., \& von Hippel, T. 1998, MNRAS, 298, 361

Becker, A. C., Silvestri, N. M., Owen, R. E., Ivezić, Ž., \& Lupton, R. H. 2007, PASP, 119,1462

Beers, T. C., \& Christlieb, N. 2005, ARA\&A, 43, 531

Beers, T. C., et al. 2000, AJ, 119, 2866

2002, AJ, 124, 931

2006, Mem. Soc. Astron. Italiana, 77, 1171

Bell, E. F., et al. 2007, ApJ, submitted (arXiv:0706.0004)

Belokurov, V., et al. 2006, ApJ, 642, L137 . 2007, ApJ, 658, 337

Bensby, T., Feltzing, S., \& Lundström, I. 2003, A\&A, 410, 527

Brook, C. B., Kawata, D., Gibson, B. K., \& Freeman, K. C. 2004, ApJ, 612, 894

Bullock, J. S., \& Johnston, K. V. 2005, ApJ, 635, 931

Carney, B. W. 1979, ApJ, 233, 211

Carollo, D., et al. 2007, Nature, 450, 1020

Casagrande, L., Portinari, L., \& Flynn, C. 2006, MNRAS, 373, 13 (CPF06)

Chiba, M., \& Beers, T. C. 2000, AJ, 119, 2843

Clem, J. L., VandenBerg, D. A., \& Stetson, P. 2008, AJ, 135, 682

Conn, B. C., et al. 2005, MNRAS, 364, L13
Covey, K., et al. 2007, AJ, 134, 2398

Dehnen, W., \& Binney, J. J. 1998, MNRAS, 298, 387

Demarque, P., \& McClure, R. D. 1980, ApJ, 242, L5

Du, C., Zhou, X., Ma, J., Shi, J., Chen, A. B., Jiang, Z., \& Chen, J. 2004, AJ, 128,2265

Duffau, S., Zinn, R., \& Vivas, A. K. 2006, ApJ, 636, L97

Eggen, O. J., Lynden-Bell, D., \& Sandage, A. R. 1962, ApJ, 136, 748

Eisenstein, D. J., et al. 2001, AJ, 122, 2267

. 2006, ApJS, 167, 40

Fan, X. 1999, AJ, 117, 2528

Feltzing, S. 2006, Mem. Soc. Astron. Italiana, 77, 1103

Finlator, K., et al. 2000, AJ, 120, 2615

Flaugher, B., et al. 2007, BAAS, 209, 22.01

Freeman, K., \& Bland-Hawthorn, J. 2002, ARA\&A, 40, 487

Fuhrmann, K. 2004, Astron. Nachr., 325, 3

Fukugita, M., Ichikawa, T., Gunn, J. E., Doi, M., Shimasaku, K., \& Schneider, D. P. $1996, \mathrm{AJ}, 111,1748$

Gilmore, G., \& Reid, N. 1983, MNRAS, 202, 1025

Gilmore, G., \& Wyse, R. F. G. 1985, AJ, 90, 2015

Gilmore, G., Wyse, R. F. G., \& Jones, J. B. 1995, AJ, 109, 1095

Gilmore, G., Wyse, R. F. G., \& Kuijken, K. 1989, ARA\&A, 27, 555

Gilmore, G., Wyse, R. F. G., \& Norris, J. E. 2002, ApJ, 574, L39

Girard, T. M., Korchagin, V. I., Caseti-Dinescu, D. I., van Altena, W. F., López, C. E., \& Monet, D. G. 2006, AJ, 132, 1768

Girardi, L., Grebel, E. K., Odenkirchen, M., \& Chiosi, C. 2004, A\&A, 422, 205

Girardi, L., \& Salaris, M. 2001, MNRAS, 323, 109

Grillmair, C. J. 2006a, ApJ, 645, L37 2006b, ApJ, 651, L29

Gunn, J. E., Knapp, G. R., \& Tremaine, S. D. 1979, AJ, 84, 1181

Gunn, J. E., et al. 1998, AJ, 116, 3040 2006, AJ, 131, 2332

Harris, W. E. 1996, AJ, 112, 1487

Helmi, A., White, S. D. M., de Zeeuw, P. T., \& Zhao, H. 1999, Nature, 402, 53 
Helmi, A., et al. 2003, ApJ, 586, 195

Hesser, J. E., et al. 1987, PASP, 99, 739

Hogg, D. W., Finkbeiner, D. P., Schlegel, D. J., \& Gunn, J. E. 2001, AJ, 122, 2129

Holberg, J. B., \& Bergeron, P. 2006, AJ, 132, 1221

Ivezić, Ž., et al. 2000, AJ, 120, 963

2003, Mem. Soc. Astron. Italiana, 74, 978

2004, Astron. Nachr., 325, 583

2005 , in ASP Conf. Ser. 338, Astrometry in the Age of the Next Generation of Large Telescopes, ed. P. K. Seidelmann \& A. K. B. Monet (San Francisco: ASP), 201

. 2006, Mem. Soc. Astron. Italiana, 77, 1057

2007a, AJ, 134, 973

2007b, in ASP Conf. Ser. 364, The Future of Photometric, Spectrophotometric and Polarimetric Standardization, ed. C. Sterken (San Francisco: ASP), 165

2008, preprint (arXiv: 0805.2366)

Jackson, T., Ivezić, Ž., \& Knapp, G. R. 2002, MNRAS, 337, 749

Jorgensen, B. R., \& Lindegren, L. 2005, A\&A, 436, 127

Jurić, M., et al. 2008, ApJ, 673, 864 (J08)

Kaiser, N., et al. 2002, Proc. SPIE, 4836, 154

Karaali, S., Bilir, S., \& Tuncel, S. 2005, Publ. Astron. Soc. Australia, 22, 24

Keller, S. C., et al. 2007, Publ. Astron. Soc. Australia, 24, 1

Kurucz, R. L. 1979, ApJS, 40, 1

Laird, J. B., Carney, B. W., \& Latham, D. W. 1988, AJ, 95, 1843

Lee, Y. S., et al. 2007a, AJ, submitted (arXiv:0710.5645) 2007b, AJ, submitted (arXiv:0710.5778)

Lenz, D. D., Newberg, J., Rosner, R., Richards, G. T., \& Stoughton, C. 1998, ApJS, 119, 121

Lupton, R. H. 1993, Statistics in Theory and Practice (Princeton: Princeton Univ. Press)

Lupton, R. H., Ivezić, Ž., Gunn, J. E., Knapp, G. R., Strauss, M. A., \& Yasuda, N. 2002, Proc. SPIE, 4836, 350

Majewski, S. R. 1992, ApJS, 78, 87 1993, ARA\&A, 31, 575

Majewski, S. R., Skrutskie, M. F., Weinberg, M. D., \& Ostheimer, J. C. 2003, ApJ, 599, 1082

Mannery, E. J., \& Wallerstein, G. 1971, AJ, 76, 890

Martin, N. F., et al. 2006, MNRAS, 367, L69

Morrison, H. L., Flynn, C., \& Freeman, K. C. 1990, AJ, 100, 1191

Munn, J. A., et al. 2004, AJ, 127, 3034

Newberg, H. J., et al. 2002, ApJ, 569, 245

Nordström, B., et al. 2004, A\&A, 418, 989

Norris, J. 1987, ApJ, 314, L39

Ojha, D. K., Bienayme, O., Robin, A. C., Creze, M., \& Mohan, V. 1996, A\&A, 311, 456

Pagel, B. E. J., \& Patchett, B. E. 1975, MNRAS, 172, 13
Penarrubia, J., et al. 2005, ApJ, 626, 128

Perryman, M. A. C., et al. 2001, A\&A, 369, 339

Pickles, A. J. 1998, PASP, 110, 863

Pier, J. R., Munn, J. A., Hindsley, R. B., Hennesy, G. S., Kent, S. M., Lupton, R. H., \& Ivezić, Ž. 2003, AJ, 125, 1559

Pourbaix, D., et al. 2005, A\&A, 444, 643

Ramírez, I., Allende Prieto, C., \& Lambert, D. L. 2007, A\&A, 465, 271

Ramírez, I., \& Meléndez, J. 2005, ApJ, 626, 465

Reddy, B. E., Lambert, D. L., \& Allende Prieto, C. 2006, MNRAS, 367, 1329

Reid, I. N., Gizis, J. E., \& Hawley, S. L. 2002, AJ, 124, 2721

Richards, G., et al. 2002, AJ, 123, 2945

Robin, A. C., Reylé, C., Derriére, S., \& Picaud, S. 2003, A\&A, 409, 523

Ryan, S. G., \& Norris, J. E. 1991, AJ, 101, 1835

Sandage, A. 1969, ApJ, 158, 1115

Sandage, A., \& Smith, L. L. 1963, ApJ, 137, 1057

Schlegel, D., Finkbeiner, D. P., \& Davis, M. 1998, ApJ, 500, 525

Schneider, D. P., et al. 2007, AJ, 134, 102

Schwarzschild, M., Searle, L., \& Howard, R. 1955, ApJ, 122, 353

Scranton, R., et al. 2002, ApJ, 579, 48

Sekiguchi, M., \& Fukugita, M. 2000, AJ, 120, 1072

Sesar, B., et al. 2007, AJ, 134, 2236

Siegel, M. H., Majewski, S. R., Reid, I. N., \& Thompson, I. B. 2002, ApJ, 578, 151

Simones, J., Newberg, H. J., \& Cole, N. 2007, BAAS, 211, 104.02

Skrutskie, M. F., et al. 2006, AJ, 131, 1163

Smith, J. A., et al. 2002, AJ, 123, 2121

Smolčić, V., et al. 2004, ApJ, 615, L141

Snider, S., Allende Prieto, C., von Hippel, T. Beers, T. C., Sneden, C., Qu, Y., \& Rossi, S. 2001, ApJ, 562, 528

Springel, V., \& Hernquist, L. 2003, MNRAS, 339, 312

Stoughton, C., et al. 2002, AJ, 123, 485

Strauss, M. A., et al. 2002, AJ, 124, 1810

Strömgren, B. 1966, ARA\&A, 4, 433

Tinsley, B. M. 1975, ApJ, 197, 159

Tucker, D., et al. 2006, Astron. Nachr., 327, 821

Tyson, J. A. 2002, Proc. SPIE, 4836, 10

VandenBerg, D. A., \& Clem, J. L. 2003, AJ, 126, 778

Vivas, A. K., \& Zinn, R. 2006, AJ, 132, 714

Vivas, A. K., et al. 2001, ApJ, 554, L33

Wallerstein, G. 1962, ApJS, 6, 407

Weinberg, M. D. 1992, ApJ, 384, 81

Wilkinson, M. I., et al. 2005, MNRAS, 359, 1306

Wyse, R. F. G. 2006, Mem. Soc. Astron. Italiana, 77, 1036

Wyse, R. F. G., \& Gilmore, G. 1995, AJ, 110, 2771

Yanny, B., et al. 2000, ApJ, 540, 825

York, D. G., et al. 2000, AJ, 120, 1579 